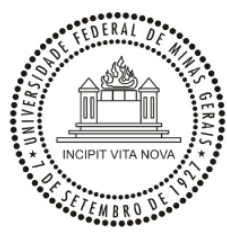

UNIVERSIDADE FEDERAL DE MINAS GERAIS - UFMG INSTITUTO DE CIÊNCIAS EXATAS DEPARTAMENTO DE PÓS-GRADUAÇÃO EM MATEMÁTICA

\title{
Isomorphism Problem for Universal Enveloping Algebras of Solvable Lie Algebras
}

\author{
José Luis Vilca Rodríguez
}

Belo Horizonte - MG

2019 


\title{
UNIVERSIDADE FEDERAL DE MINAS GERAIS - UFMG INSTITUTO DE CIÊNCIAS EXATAS DEPARTAMENTO DE PÓS-GRADUAÇÃO EM MATEMÁTICA
}

\author{
José Luis Vilca Rodríguez
}

\author{
Advisor: Csaba Schneider
}

\section{Isomorphism Problem for Universal Enveloping Algebras of Solvable Lie Algebras}

Thesis presented to the Federal University of Minas Gerais in fulfillment of the requirements for the degree of Doctor of Philosophy in Mathematics

Belo Horizonte - MG 
Siempre que biene el tiempo fresco, o sea al medio del otonio, a mí me da la loca de pensar ideas de tipo eséntrico y esótico, como ser por egenplo que me gustaría venirme golondrina para agarrar y volar a los paíx adonde haiga calor, o de ser hormiga para meterme bien adentro de una cueva y comer los productos guardados en el verano o de ser una bívora como las del solójico, que las tienen bien guardadas en una jaula de vidrio con calefación para que no se queden duras de frío, que es lo que les pasa a los pobres seres humanos que no pueden comprarse ropa con lo cara questá, ni pueden calentarse por la falta del querosén, la falta del carbón, la falta de lenia, la falta de petrolio y tamién la falta de plata, porque cuando uno anda con biyuya ensima puede entrar a cualquier boliche y mandarse una buena grapa que hay que ver lo que calienta, aunque no conbiene abusar, porque del abuso entra el visio y del visio la dejeneradés tanto del cuerpo como de las taras moral de cada cual, y cuando se viene abajo por la pendiente fatal de la falta de buena condupta en todo sentido, ya nadie ni nadies lo salva de acabar en el más espantoso tacho de basura del desprastijio humano, y nunca le van a dar una mano para sacarlo de adentro del fango enmundo entre el cual se rebuelca, ni más ni meno que si fuera un cóndor que cuando joven supo correr y volar por la punta de las altas montanias, pero que al ser viejo cayó parabajo como bombardero en picada que le falia el motor moral. iY ojalá que lo que estoy escribiendolé sirbalguno para que mire bien su comportamiento y que no searrepienta cuando es tarde y ya todo se haiga ido al corno por culpa suya!

CÉSAR BRUTO, Lo que me gustaría ser a mí si no fuera lo que soy.

Sí, ya sé, hay más problemólogos que solucionólogos, pero ¿qué vamos a hacerle? QUINO, Mafalda. 


\section{Agradecimientos}

El presente trabajo es el fruto de significativos esfuerzos de muchas personas. Juntos pudimos concretar la fabricación de esta pequeña pieza, en la gran maquina que es la matemática. Así como una pared no se construye sólo con ladrillos, este trabajo no se construyo sólo con investigación, hizo falta la mano de muchas personas que me brindaron apoyo y motivación a lo largo de mi vida. Llegó la hora de agradecer a todos ellos.

- A mis padres. Gracias por transmitirme lo importante que es la educación y apoyarme siempre en todas mis decisiones. Gracias por lo mucho que se sacrificaron por mí, para que pueda tener las oportunidades que ustedes no tuvieron. La conclusión de este doctorado es también la conclusión de sus esfuerzos.

- A mis hermanos. Por la camaradería, apoyo, motivación y confianza en todos estos años fuera de casa, y por todas las vivencias en todo el tiempo que estuve físicamente más cerca de ustedes. Ustedes son mis mejores amigos. Para ustedes mis agradecimientos.

- A mis abuelos, Leoncio y Valeriana. Gracias por todo el tiempo que me dedicaron y por el apoyo incondicional que me brindaron.

- A mi orientador, el profesor Csaba Schneider. Gracias por la dedicación, tiempo, esfuerzo y paciencia para orientarme en mi vida profesional. Creo que debe ser muy difícil orientarme. Aprendí mucho con usted.

- A los profesores que examinaron este trabajo: Ana Cristina, André Contiero, Lucas Calixto, Víctor Petrogradsky y Wagner Cortes. Gracias por el tiempo que dedicaron a leer este trabajo, y por las muchas sugerencias y contribuciones que sirvieron para enriquecerlo.

- Al profesor Walter Torres, uno de mis grandes mentores. El único profesor que sin usar una pizarra me hizo entender la matemática. Gracias por siempre motivarme a estudiar matemática. 
- A la CNPq y la CAPES por el apoyo financiero.

- A los funcionarios de la secretaria. En especial a Andrea y Kelli. Gracias por ayudarme en todos los momentos de correría. Siempre me atendieron con amabilidad y eficiencia, y sabían más de mi vida académica que yo mismo.

- A los amigos que conocí dentro y fuera de mi vida académica. A Carlos (papa frita), Eduardo (manito), Leo (el psicólogo), Jhon (uai fai), Katy (la tata), Simeona (ya se verá...), gracias a ustedes por más de una década de amistad. A Ricardo (Homer), Joel (Desmond), Carlos Salazar (Andi), Alfonso (Matute), Matute (Alfonso), Tauan (baiano), Mario, Rafael, Vinicius, Ayane (Hahihani), Myrla (aTESTAda), Diogo (tá maluco?), Moacir (Moacicinho), Aislan (pisado), Eduardo (Gordon), Aline (vamos pro forró?), Rensso, Guillermo, Víctor, Andrea, Elena. Gracias a todos ustedes por mostrarme que lejos de casa amigo es sinónimo de familia. 


\begin{abstract}
We dedicate this thesis to study the isomorphism problem for solvable Lie algebras of dimension at most 4 . The principal contribution is to show that the isomorphism problem has a positive solution for 4-dimensional solvable Lie algebras over a field of characteristic zero. That is, we show that given two 4-dimensional Lie algebras $L$ and $H$ over a field $\mathbb{F}$ of characteristic 0 , then $U(L) \cong U(H)$ imply $L \cong H$.

To reach our goal we use three tools. The first is the Frobenius semiradical and its relationship with the structure of a universal enveloping algebra of a Lie algebra. Using the Frobenius semiradical we show that for certain Lie algebras $L$ the dimension of $Z(L)$ is determined by the isomorphism type of $U(L)$. The second tool is a construction. Given a Lie algebra $L$ and an abelian ideal $M$ of $L$, we produce a Lie algebra $L_{M}$ which is invariant under a group of automorphisms of $U(L)$. We show that in several cases the Lie algebras $L$ and $L_{M}$ are isomorphic. Finally, the third tool is the study of structure of the universal enveloping algebras for Lie algebras with an ideal of codimension one. Analysing this type of Lie algebras we show that the isomorphism type of $L / L^{\prime \prime}$ is determined by the isomorphism type of $U(L)$, and as a consequence of this fact the isomorphisms problem has a positive solution for metabelian Lie algebras in which the codimension of the derived algebra is one.

Although our main result is given in dimension four, in several of our results we require only that the Lie algebra be finite-dimensional. Further, many of our results hold for an arbitrary base field independently of the characteristic.
\end{abstract}

Key words: Solvable Lie algebras, universal enveloping algebras, isomorphism problem. 


\section{Resumo}

Dedicamos esta tese, basicamente, a estudar o problema do isomorfismo para álgebras de Lie solúveis de dimensão no máximo 4. A principal contribuição é mostrar que o problema do isomorfismo tem uma solução positiva para álgebras de Lie solúveis de dimensão 4 sobre um corpo de característica zero. Isto é, mostramos que, dadas duas álgebras de Lie 4-dimensionais $L$ e $H$ sobre um corpo $\mathbb{F}$ de característica 0 , então $U(L) \cong U(H)$ implica $L \cong H$.

Para alcançar nosso objetivo, usamos basicamente três ferramentas. A primeira é o semirradical de Frobenius e sua relação com a estrutura de uma álgebra envolvente universal de uma álgebra de Lie. Usando o semirradical de Frobenius, mostramos que, para certas álgebra de Lie $L$, a dimensão de $Z(L)$ é determinada pelo tipo de isomorfismo de $U(L)$. A segunda ferramenta é uma construção. Dada uma álgebra de Lie $L$ e um ideal abeliano $M$ de $L$, produzimos uma álgebra de Lie $L_{M}$ que é invariante por um grupo de automorfismos de $U(L)$. Mostramos que, em vários casos, as álgebras de Lie $L$ e $L_{M}$ são isomorfas. Finalmente, a terceira ferramenta é o estudo da estrutura das álgebras envolventes universais para álgebras de Lie com um ideal de codimensão um. Analisando este tipo de álgebras de Lie, mostramos que o tipo de isomorfismo $L / L^{\prime \prime}$ é determinado pelo tipo de isomorfismo de $U(L)$, e como consequência deste fato temos que o problema dos isomorfismos tem uma solução positiva para álgebras de Lie metabelianas para as quais a codimensão da álgebra derivada é um.

Embora nosso principal resultado seja dado em dimensão 4, em vários dos nossos resultados, exigimos apenas que a álgebra de Lie seja de dimensão finita. Além disso, muitos dos nossos resultados valem para um corpo arbitrário, independentemente da característica.

Palavras Chave: Álgebras de Lie solúveis, álgebras envolventes universais, problema do isomorfismo. 


\section{Contents}

\begin{tabular}{lr}
\hline Resumo Estendido & 8
\end{tabular}

$\begin{array}{lll}1 & \text { Introduction } & 17\end{array}$

1.1 Historical background $\ldots \ldots \ldots \ldots$. . . . . . . . . . . . 17

1.2 Center, semicenter and Frobenius semiradical $\ldots \ldots \ldots \ldots . . . .21$

$1.3 \quad$ Structure of $U(L)$ for certain Lie algebras. . . . . . . . . . . . . 23

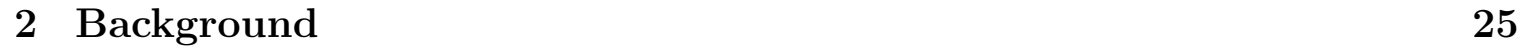

$2.1 \quad$ Lie algebras and their universal enveloping algebras $\ldots \ldots \ldots \ldots 25$

2.2 Ring of quotients of the universal enveloping algebra $\ldots \ldots \ldots . \quad 36$

$\begin{array}{lll}2.3 & \text { Isomorphism between universal enveloping algebras of Lie algebras . . } 39\end{array}$

2.4 Hopf algebras $\ldots \ldots \ldots \ldots \ldots$

\begin{tabular}{|lll}
\hline 3 & Solvable Lie algebras of dimension at most 4 & 47
\end{tabular}

3.1 The classification by de Graaf $\ldots \ldots \ldots \ldots . \ldots . \ldots 47$

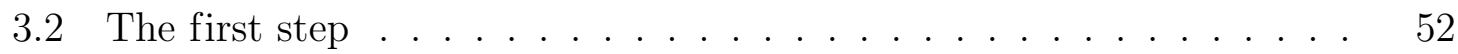

4 Center, semicenter and Frobenius semiradical 54

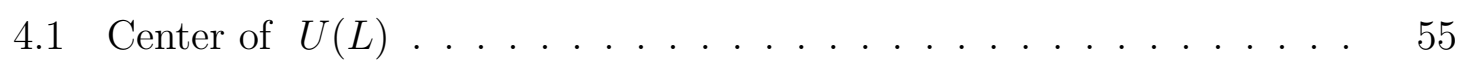

4.2 A construction of counterexamples for the isomorphism problem . . . 57

4.3 Semicenter of $U(L) \ldots \ldots \ldots \ldots$

4.4 The Frobenius Semiradical of a Lie algebra . . . . . . . . . . . . 63

\begin{tabular}{|lll}
5 & Universal enveloping algebras of certain Lie algebras & 71
\end{tabular}

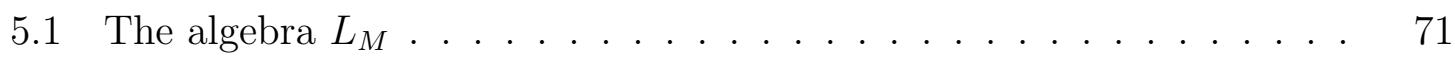

5.2 Computing $L_{M}$ for some Lie algebras $\ldots \ldots \ldots \ldots \ldots \ldots$ 
6 Solvable Lie algebras with an ideal of codimension one 85

6.1 Lie algebras with ideals of codimension $1 \ldots \ldots \ldots . . . \ldots 5$

6.2 The final part of the proof of Theorem $3.2 .1 \ldots \ldots \ldots$. . . . . 91

\begin{tabular}{ll}
\hline Bibliography & 93
\end{tabular} 


\section{Resumo Estendido}

\section{Antecedentes Históricos}

O estudo das álgebras de Lie tornou-se tão importante quanto natural em algumas áreas da matemática, como geometria e álgebra. Estas estruturas algébricas aparecem quando estudamos campos de vetores de certas variedades diferenciáveis chamadas grupos de Lie. Isto dá uma dualidade entre geometria e álgebra, mas devido à sua riqueza estrutural, as álgebras de Lie são estudadas independentemente da geometria, e esta é uma área de interesse independente em matemática.

Uma álgebra de Lie $L$ é, em geral, uma estrutura não-associativa. Entretanto é possível associar tal álgebra a uma álgebra associativa $U(L)$, que verifica uma certa propriedade universal e contém $L$, como subalgebra de Lie. Esta álgebra associativa é chamada álgebra envolvente universal. A principal motivação desta tese é a questão que segue.

O problema do isomorfismo para álgebras envelopantes universais de álgebras de Lie: Se $L, H$ são álgebras de Lie, $U(L) \cong U(H)$ implica que $L \cong H$ ?

Não sabemos exatamente quem enunciou este problema pela primeira vez nem quando foi enunciado. Porém, a referência mais antiga que temos, é o artigo de Bergman [1], onde ele enunciou este problema antes da Seção 4. Nesta tese, este problema será referido como o problema do isomorfismo .

Acreditamos que o problema do isomorfismo tem suas raízes históricas em um famoso problema da teoria de grupos, a saber, o problema do isomorfismo para anéis de grupos. Tal problema se pergunta, para dois grupos $G$ e $H$, se o isomorfismo dos anéis $\mathbb{Z} G \cong \mathbb{Z} H$ implica o isomorfismo $G \cong H$. Este problema foi introduzido 
por Higman na sua tese doutoral em 1940. Muitos resultados que são verdadeiros para anéis de grupo, também são verdadeiros para álgebras envolventes universais de álgebras de Lie, cada um em sua própria linguagem. Por exemplo, o tipo de isomorfismo de um grupo nilpotente $G$ de classe no máximo dois é determinado pelo tipo de isomorfismo $\mathbb{Z} G$, veja [22]. Analogamente, em [27, Corolário 5.3], é mostrado que o tipo de isomorfismo de uma álgebra de Lie nilpotente $L$ de classe no máximo dois é determinado por sua álgebra envolvente universal $U(L)$. Isso nos leva a suspeitar que existe uma relação entre esses dois problemas. De fato, o artigo de Quillen [25] exibe um isomorfismo entre algumas álgebras envolventes universais restritas e certas álgebras graduadas de anéis de grupo, relacionando esses dois problemas.

Em geral, o problema do isomorfismo tem resposta negativa. Isso significa que existem álgebras de Lie que não são isomorfas, mas tem álgebras envolventes universais isomorfas, veja o Exemplo 2.1.6 no Capítulo 2. No entanto, existem várias classes de álgebras de Lie nas quais o problema do isomorfismo tem solução positiva. Introduzimos uma linguagem com o propósito de apresentar de forma compacta os principais resultados sobre o problema do isomorfismo que são conhecidos na literatura. Seja $\mathcal{C}$ uma classe de álgebras de Lie. Dizemos que o problema do isomorfismo tem solução positiva em $\mathcal{C}$ se $U(L) \cong U(H)$ implica $L \cong H$ para todos $L, H \in \mathcal{C}$. Com esta linguagem, enunciamos o seguinte teorema.

Teorema. O problema do isomorfismo tem uma solução positiva nas seguintes classes de álgebras de Lie:

1. Álgebras de Lie abelianas de dimensão finita.

2. Álgebras de Lie de dimensão no máximo três sobre corpos de característica diferente de dois.

3. Álgebras de Lie nilpotentes de classe no máximo dois.

4. Álgebras de Lie nilpotentes livres finitamente geradas.

5. Álgebras de Lie nilpotentes de dimensão no máximo quatro.

6. Álgebras de Lie nilpotentes de dimensão cinco sobre corpos de característica diferente de dois. 
7. Álgebras de Lie nilpotentes de dimensão seis sobre corpos de característica diferente de dois e três.

O item 1 do teorema anterior é bem conhecido e será mostrado em uma discussão após o Lema 2.3.3. Malcolmson, em 1992, mostrou que se $L$ é uma álgebra de Lie, simples e de dimensão 3 sobre um corpo de característica diferente de dois, então a forma de Killing de $L$ pode ser usada para determinar o tipo de isomorfismo de $L$ e também o de $U(L)$, veja [15]. Como consequência do resultado de Malcolmonson, o problema do isomorfismo tem uma solução positiva para álgebras de Lie simples de dimensão 3 sobre um corpo de característica diferente de dois. Mais tarde, em 2004, Chun, Kajiwara e Lee provaram que o problema do isomorfismo tem uma solução positiva para álgebras de Lie não simples e de dimensão 3 sobre um corpo arbitrário. Eles combinaram seu resultado com o resultado de Malcolmson em [4] e completaram a prova do item 2. No Capítulo 5 desta tese, inspirados nas técnicas de Chun, Kajiwara e Lee, introduzimos uma álgebra de Lie que é invariante por um grupo de automorfismos da álgebra envolvente universal. Em 2007, Riley e Usefi mostraram os itens 3 e 4 em [27]. O artigo de Riley e Usefi [27] é, em minha opinião, um dos trabalhos mais completos, até o momento, sobre o problema do isomorfismo. O item 5 é uma consequência da classificação de de Graaf [5] e alguns resultados de Riley e Usefi em [27]. Finalmente, em 2011 os itens 6 e 7 foram mostrados por Schneider e Usefi em [29], onde os autores usaram a classificação dada por de Graaf para álgebras de Lie nilpotentes de dimensão no máximo 6 (veja [6]), e eles dividiram essas álgebras de Lie em grupos de acordo com os tipos de isomorfismo de suas álgebras de Lie graduadas associadas. Depois, eles analisaram o problema do isomorfismo em cada grupo. As restrições sobre a característica são necessárias, como foi mostrado por exemplos no mesmo artigo. Em muitos casos, tais exemplos têm uma razão comum que será destacada no Capítulo 4 desta tese.

Embora o problema do isomorfismo tenha, em geral, uma solução negativa, várias propriedades de uma álgebra de Lie são determinadas pelo tipo de isomorfismo de sua álgebra envolvente universal. Por exemplo, é bem conhecido que se $L$ é uma álgebra de Lie de dimensão finita, então a dimensão (linear) de $L$ coincide com a dimensão de Gelfand-Kirillov de $U(L)$ (veja [16, 8.1 .15 (iii)]); assim, $\operatorname{dim} L$ é determinada apenas pelo tipo de isomorfismo $U(L)$. O resultado mais surpreendente nessa direção diz que 
dada uma álgebra de Lie $L$, a álgebra de Lie graduada $\operatorname{gr}(L)=\bigoplus_{i \geqslant 1} \gamma_{i}(L) / \gamma_{i+1}(L)$ é determinada pelo tipo de isomorfismo $U(L)$, veja [27, Proposição 4.1]. Riley e Usefi em 27] apresentaram um estudo detalhado de algumas propriedades das álgebras de Lie que são determinadas pelo tipo de isomorfismo da sua álgebra envolvente universal. No seguinte teorema, apresentamos um resumo das propriedades mais importantes que são determinadas pelo tipo de isomorfismo da álgebra envolvente universal.

Teorema ([27]). Seja L e $H$ álgebras de Lie e $\alpha: U(L) \rightarrow U(H)$ um isomorfismo de álgebras, suponha que L tem dimensão finita. Então, se verifica o seguinte.

1. $\operatorname{dim} L=\operatorname{dim} H e \operatorname{dim} L / L^{\prime}=\operatorname{dim} H / H^{\prime}$.

2. Se L é nilpotente, então também o é H. Além disso, neste caso, as classes de nilpotência de $L$ e $H$ coincidem.

3. $L^{\prime} / L^{\prime \prime} \cong H^{\prime} / H^{\prime \prime}$; em particular, $\operatorname{dim} L^{\prime} / L^{\prime \prime}=\operatorname{dim} H^{\prime} / H^{\prime \prime}$.

4. Se L é metabeliana, então também o é $H$.

5. Se L é solúvel, então também o é $H$.

6. Se $M$ e $N$ são ideais de $L$ e $H$, respectivamente; tal que $\alpha(M U(L))=N U(H)$. Então, $M / M^{\prime} \cong N / N^{\prime}$.

As propriedades que são determinadas pelo tipo de isomorfismo da álgebra envolvente universal desempenham um papel importante no estudo do problema do isomorfismo. Mais precisamente, uma maneira de encontrar mais classes de álgebras de Lie nas quais o problema do isomorfismo tem solução positiva é encontrar propriedades que são determinadas pelo tipo de isomorfismo da álgebra envolvente universal e analisar essas propriedades para definir essas classes. Por exemplo, Usefi em [27, Proposição 5.2] mostrou que o tipo de isomorfismo $L / \gamma_{3}(L)$ é determinado pelo tipo de isomorfismo $U(L)$. Assim, se $\mathcal{C}$ é a classe de álgebras de Lie nilpotentes da classe de nilpotência dois (i.e. $\gamma_{3}(L)=0$ ), temos que o problema do isomorfismo tem solução positiva em $\mathcal{C}$. Um dos objetivos desta tese é encontrar algumas propriedades que são determinadas pelo tipo de isomorfismo das álgebras envolventes 
universais, e logo encontrar algumas classes das álgebras de Lie nas quais o problema do isomorfismo tem solução positiva.

Nossa principal contribuição para o problema do isomorfismo é provar o seguinte teorema.

Teorema 3.2.1(Teorema principal) Sejam L e $H$ álgebras de Lie solúveis e de dimensão 4 sobre um corpo de característica 0 . Se $U(L) \cong U(H)$, então $L \cong H$.

A prova do Teorema 3.2.1 consiste basicamente em duas etapas. Primeiro, usando a classificação de de Graaf, dividimos as álgebras de Lie em cinco grupos (essa divisão pode ser encontrada na Seção 3.1 de forma que duas álgebras de Lie em diferentes grupos não podem ter álgebras envolventes universais isomorfas. Segundo, analisamos cada grupo para garantir que no mesmo grupo não existam duas álgebras de Lie não isomorfas com álgebras envolventes universais isomorfas. Esta é a parte crucial (e mais complicada) da prova. O segundo passo da prova do Teorema 3.2.1 está espalhado em várias partes nos Capítulos 4, 5 e 6. Em cada um dos capítulos desenvolvemos várias técnicas que nos ajudam na segunda etapa da prova do Teorema 3.2.1.

Este teorema é o resultado mais importante desta tese e todos os resultados aqui foram motivados para tentar encontrar uma prova deste teorema. No entanto, nem todos os nossos resultados têm uma relação direta com o Teorema 3.2.1, mas acreditamos que todos eles foram importantes para entender melhor o problema do isomorfismo e algumas questões relacionadas a este. Resumimos nossos principais resultados nas próximas duas seções.

\section{Centro, Semicentro e Semirradical de Frobenius}

As primeiras seções do Capítulo 4 estão dedicadas ao estudo do centro e a um resultado que nos ajuda a dar contraexemplos para o problema do isomorfismo. Este resultado foi inspirado pela análise de vários exemplos do artigo de Usefi e Schneider [29]. No teorema a seguir, reunimos o Teorema 4.2.1 e o Corolário 4.2.2, que são os dois principais resultados nessa direção. Dada uma álgebra de Lie $L$, o conjunto de elementos primitivos de $U(L)$ é denotado por $\mathcal{P}(U(L))$, veja a Seção 2.4. O produto direto e semidireto de álgebras de Lie é definido antes do Lema 2.1.3. 
Teorema. Sejam $L=M \oplus\langle x\rangle$ e $H=N \rtimes\langle y\rangle$ duas álgebras de Lie nilpotentes de dimensão finita, onde $M$ e $N$ são ideais de $L$ e $H$, respectivamente. Suponha que $M \cong$ $N$ e $\operatorname{gr}(L) \cong \operatorname{gr}(H)$, e que exista um elemento $u \in U(N)$ tal que $\left(\operatorname{ad}_{U(H)} u\right)(N) \subseteq N$ $\left.e \operatorname{ad}_{U(H)} u\right|_{N}=\left.\operatorname{ad}_{U(H)} y\right|_{N}$. Então, existe um isomorfismo entre $U(L)$ e $U(H)$. Além disso, se $u \in \mathcal{P}(U(N))$ então $U(L) \cong U(H)$ como bialgebras.

No Capítulo 4 veremos como podemos usar este teorema para produzir contraexemplos para o problema do isomorfismo em característica prima. No momento, não encontramos um par de álgebras de Lie que satisfaçam as condições do teorema acima em característica zero. De fato, em característica zero, o problema do isomorfismo ainda é um problema aberto, em geral.

No Capítulo 4 também definimos o semicentro $S z(U(L))$ de $U(L)$, veja Seção 4.3 . Outro fato importante mostrado no Capítulo 4 é o Teorema 4.3.3, o qual basicamente diz que $S z(U(L))$ é invariante por isomorfismos entre álgebras envolventes universais.

Na seção anterior vimos vários invariantes de álgebras de Lie que são determinados pelo tipo de isomorfismo da álgebra envolvente universal. É interessante notar que a dimensão do centro não aparece entre esses invariantes. De fato, exemplos mostram que se char $\mathbb{F}$ é um primo então existem álgebras de Lie $L$ e $H$ tais que $U(L) \cong U(H)$, mas $\operatorname{dim} Z(L) \neq \operatorname{dim} Z(H)$ (ver Exemplo 2.1.6). Por outro lado, nenhum desses exemplos existe na característica zero, e conjectura-se que em característica zero a dimensão do centro de $L$ é determinada pelo tipo de isomorfismo de $U(L)$. Inspirado por esta conjectura, na Seção 4.4 estudamos o semirradical de Frobenius $F(L)$ de uma álgebra de Lie $L$, veja a Seção 4.4 para a definição de $F(L)$. Este objeto foi introduzido por Ooms em 21] e é muito importante para entender a estrutura de $U(L)$. Em particular, estudamos as álgebras de Lie quadrado integráveis, que por definição são as álgebras de Lie $L$ tais que $F(L)=Z(L)$. Mais precisamente, mostraremos os seguintes teoremas.

Teorema 4.4.6 Suponha que L seja uma álgebra de Lie quadrado integrável de dimensão finita sobre um corpo $\mathbb{F}$ da característica 0. Então $U(Z(L))=Z(U(L))$.

Teorema 4.4.8 Suponha que L e H sejam álgebras de Lie quadrado integráveis de dimensão finita sobre um corpo $\mathbb{F}$ de característica 0 , e que $\varphi: U(L) \rightarrow U(H)$ é um isomorfismo de álgebra. Então, $\operatorname{dim} Z(L)=\operatorname{dim} Z(H)$. 
Outro resultado importante no Capítulo 4 é o Teorema 4.4.10. Este teorema descreve todas as álgebras de Lie solúveis de dimensão 4 que são quadrado integráveis. Para a prova do Teorema 4.4.10 usamos a classificação dada por de Graaf [5]. Como consequência direta do Teorema 4.4.10, calculamos o centro das álgebras envolventes universais para álgebras de Lie solúveis quadrado integráveis de dimensão 4 sobre um corpo de característica 0. Estudar o centro de álgebras envolventes universais tem um interesse independente. De fato, o centro de $U(L)$ desempenha um papel importante na teoria de representações de $L$; veja por exemplo [8, Capítulo 4] e [30, Seção 5.1].

\section{Estrutura de $U(L)$ para certas álgebras de Lie}

Na Seção 5.1 apresentamos uma construção. Para uma álgebra de Lie $L$, um ideal abeliano $M$ de $L$ e um ideal maximal (bilateral) $\mathfrak{m}$ de $U(L)$, tal que $U(L) / \mathfrak{m} \cong$ $\mathbb{F}$, construímos outra álgebra de Lie $L_{M}$ que é invariante por um certo grupo de automorfismos de $U(L)$.

Um dos resultados mais importantes na Seção 5.1 é o Teorema 5.1.4, que fornece uma condição para o tipo de isomorfismo de $L_{M}$ ser determinado pelo tipo de isomorfismo de $U(L)$. Consequentemente, temos que a álgebra de Lie $L_{M}$ é invariante por um certo grupo de automorfismos de $U(L)$.

Teorema 5.1.4 Sejam L e $H$ álgebras de Lie de dimensão finita sobre um corpo arbitrário $\mathbb{F}$ e sejam $M$ e $N$ ideais abelianos de $L$ e $H$, respectivamente. Suponha que $\alpha: U(L) \rightarrow U(H)$ é um isomorfismo de álgebras tal que $\alpha(M U(L))=N U(H)$. Seja $\mathfrak{m}$ um ideal maximal (bilateral) de $U(L)$ tal que $U(L) / \mathfrak{m} \cong \mathbb{F}$ e $M \subseteq \mathfrak{m}$ e seja $\mathfrak{n}=\alpha(\mathfrak{m})$. Então $\alpha$ induz um isomorfismo entre as álgebras de Lie $L_{M}$ e $H_{N}$.

Outro resultado importante do Capítulo 5 é a Proposição 5.2.1, que é um resultado parcial para a segunda etapa da prova do Teorema 3.2.1.

No Capítulo 6, estudamos a estrutura da álgebra envolvente universal $U(L)$ para álgebras de Lie da forma $L=M \rtimes\langle x\rangle$, onde $M$ é um ideal. Estas álgebras de Lie têm algumas vantagens. Primeiro, o complemento (como espaços vetoriais) de $M$ é uma álgebra de Lie; em geral, isso não é verdade se $\operatorname{dim}(L / M) \geqslant 2$. Segundo, $U(L / M)$ pode ser visto como o anel de polinômios $\mathbb{F}[x]$; e os automorfismos de $\mathbb{F}[x]$ são bem compreendidos. Nós vamos explorar isso. 
Um dos resultados mais importantes no Capítulo 6 é a Proposição e o corolário imediato desta, enunciados a seguir. Estes foram inspirados por um resultado correspondente para anéis de grupo, originalmente provado por Whitcomb em [33], veja também [24, Teorema 9.3.13]. Com este resultado, Whitcomb mostrou que o problema do isomorfismo para anéis de grupos tem uma solução positiva para grupos metabelianos.

Proposição 6.1.1 Sejam $L=M \rtimes\langle x\rangle$ e $H=N \rtimes\langle y\rangle$ álgebras de Lie de dimensão finita sobre um corpo arbitrário, onde $M$ e $N$ são ideais de $L$ e $H$, respectivamente. Suponha que $\alpha: U(L) \rightarrow U(H)$ é um isomorfismo de álgebras tal que $\alpha(M U(L))=$ $N U(H)$. Então, $L / M^{\prime} \cong H / N^{\prime}$.

Corolário 6.1.2 Suponha que L, $H, N, M$ são como na Proposição 6.1.1 e que $\alpha$ : $U(L) \rightarrow U(H)$ é um isomorfismo de álgebras. Então o seguinte é válido.

1. Se $\alpha(M U(L))=N U(H)$ e $M$ ou $N$ é abeliano, então $L \cong H$.

2. Se $M=L^{\prime}$ e $N=H^{\prime}$, então $L / L^{\prime \prime} \cong H / H^{\prime \prime}$. Em particular; se $L$ ou $H$ é metabeliano, $L \cong H$.

Como dito na primeira seção, um dos objetivos desta tese é encontrar as propriedades de uma álgebra de Lie $L$ que são determinadas pelo tipo de isomorfismo de $U(L)$, e usar estas propriedades para definir uma classe na qual o problema do isomorfismo tem solução positiva. Vejamos que podemos fazer isto com o item (2) do Corolário 6.1.2. De fato, do Corolário 6.1.2 (2) temos que o tipo de isomorfismo $L / L^{\prime \prime}$ é determinado pelo tipo de isomorfismo $U(L)$. Assim, se $\mathcal{C}$ é a classe de álgebras de Lie metabelianas de dimensão finita com subálgebra derivada de codimensão 1, então o problema do isomorfismo tem solução positiva em $\mathcal{C}$.

Outro resultado do Capítulo 6 que merece ser mencionado é a Proposição 6.1.4. que indicamos a seguir. Esta é importante porque é usada em um resultado parcial para o segundo passo da prova do Teorema 3.2.1.

Proposição 6.1.4 Suponha que L e $H$ são duas álgebras de Lie metabelianas de dimensão finita sobre um corpo $\mathbb{F}$ de característica 0 , e que $L=\left(L^{\prime}+Z(L)\right) \rtimes\langle x\rangle$ e $H=(H+Z(H)) \rtimes\langle y\rangle$. Se $\alpha: U(L) \rightarrow U(H)$ é um isomorfismo de álgebra, então $L \cong H$. 
Na prova desta proposição, a hipótese de que a característica do corpo é zero é necessária, uma vez que usamos um resultado provado por Ooms [21], que é válido apenas neste caso.

No Capítulo 6, também apresentamos outras proposições que são usadas no segundo passo da prova do Teorema 3.2.1 e no final da prova do Teorema 3.2.1. Este passo final consiste em reunir os resultados parciais mostrados nos capítulos anteriores. 


\section{Chapter 1}

\section{Introduction}

\subsection{Historical background}

The study of Lie algebras became as important as it is natural in some areas of mathematics such as geometry and algebra. These algebraic structures appear when studying vector fields of certain differential manifolds called Lie groups. This gives a duality between geometry and algebra, but due to its structural richness, Lie algebras are studied independently of geometry, and this is an area of independent interest in mathematics.

A Lie algebra $L$ is a non-associative structure, but it is possible to associate uniquely an associative algebra $U(L)$ which verifies a certain universal property and contains $L$ as Lie subalgebra. This associative algebra is called universal enveloping algebra. The main motivation of this thesis is the following question.

The isomorphism problem for universal enveloping algebras of Lie algebras: If $L, H$ are Lie algebras, does $U(L) \cong U(H)$ imply that $L \cong H$ ?

We do not know exactly who stated this problem for the first time or when it was stated. But the oldest reference that we know is Bergman's paper [1], where he stated this problem before Section 4. In this thesis this problem will be referred to as the isomorphism problem.

We believe that the isomorphism problem has its historical roots in a famous problem of group theory, namely the isomorphism problem for integral group rings. This problem asks, for two groups $G$ and $H$, if the ring isomorphism $\mathbb{Z} G \cong \mathbb{Z} H$ implies the 
isomorphism $G \cong H$. This problem was introduced by Higman in his doctoral thesis in 1940. Many results that are true for group rings are true for universal enveloping algebras of Lie algebras too, of course, each in its own language. For example, the isomorphism type of a nilpotent group $G$ of class at most two is determined by the isomorphism type of $\mathbb{Z} G$, see $[22]$. Analogously, in [27, Corollary 5.3] it is shown that the isomorphism type of a nilpotent Lie algebra $L$ of class at most two is determined by its universal enveloping algebra $U(L)$. This leads us to suspect that there is a relationship between these two problems. In fact, the paper of Quillen [25] gives an isomorphism between some restricted universal enveloping algebras and certain graded algebras of group rings, relating these two problems.

In general, the isomorphism problem has a negative answer, which means that there are non-isomorphic Lie algebras with isomorphic universal enveloping algebras, see Example 2.1.6 in Chapter 2. However, there exist several classes of Lie algebras in which the isomorphism problem has a positive solution. We introduce a language with the purpose of stating in a compact way the principal results about the isomorphism problem which are known in the literature. Let $\mathcal{C}$ be a class of Lie algebras. We say that the isomorphism problem has a positive solution in $\mathcal{C}$ if $U(L) \cong U(H)$ implies $L \cong H$ for all $L, H \in \mathcal{C}$. With this language we state the following theorem.

Theorem. The isomorphism problem has a positive solution in the following classes of Lie algebras:

1. Finite-dimensional abelian Lie algebras.

2. Lie algebras of dimension at most three over fields of characteristic different from two.

3. Nilpotent Lie algebras of class at most two.

4. Finitely generated free nilpotent Lie algebras.

5. Nilpotent Lie algebras of dimension at most four.

6. Nilpotent Lie algebras of dimension five over fields of characteristic different from two. 
7. Nilpotent Lie algebras of dimension six over fields of characteristic different from two and three.

Item 1 of the previous theorem is well-known and will be shown in a discussion after Lemma 2.3.3. Malcolmson in 1992 showed that if $L$ is a simple 3-dimensional Lie algebra over a field of characteristics different from two, then the Killing form of $L$ can be used to determine the isomorphism type of $L$ and also $U(L)$, see [15]. As a consequence of the result by Malcolmson the isomorphism problem has a positive solution for simple Lie algebras of dimension 3 over a field of characteristic different from two. Later, in 2004 Chun, Kijiwara and Lee proved that the isomorphism problem has a positive solution for 3-dimensional non-simple Lie algebras over an arbitrary field. They combined their result with the result by Malcolmson in [4] and completed the proof of item 2. In Chapter 5 of this thesis, inspired by the techniques of Chun, Kijiwara and Lee we introduced a Lie algebra which is invariant under a group of automorphisms of the universal enveloping algebra. In 2007 Riley and Usefi showed items 3 and 4 in [27]. The paper by Riley and Usefi [27] is, in my opinion, one of the most complete treaties to date on the isomorphism problem. Item 5 is a consequence of the classification by de Graaf [5] and some results by Riley and Usefi in [27]. Finally, in 2011 items 6 and 7 were shown by Schneider and Usefi in [29], where the authors used the classification given by de Graaf for nilpotent Lie algebras of dimension at most 6 (see [6]), and they divided these Lie algebras into groups according to the isomorphism types of their associated graded Lie algebras. Then they analyzed the isomorphism problem in each group. The restrictions on the characteristic are necessary as shown by examples of the same paper. In many cases, such examples have a common underlying reason that will be highlighted in Chapter 4 of this thesis.

Although the isomorphism problem has in general a negative solution, several properties of a Lie algebra are determined by the isomorphism type of its universal enveloping algebra. For instance, it is well known that if $L$ is a finite-dimensional Lie algebra, then the (linear) dimension of $L$ coincides with the Gelfand-Kirillov dimension of $U(L)$ (see $[16,8.1 .15$ (iii)]); thus $\operatorname{dim} L$ is determined only by the isomorphism type of $U(L)$. The most surprising result in this direction says that given a Lie algebra $L$, the graded Lie algebra $\operatorname{gr}(L)=\bigoplus_{i \geqslant 1} \gamma_{i}(L) / \gamma_{i+1}(L)$ is determined by the isomorphism type of $U(L)$, see [27, Proposition 4.1]. Riley and Usefi in 27] presented a detailed 
study of some properties of Lie algebras that are determined by the isomorphism type of its universal enveloping algebra; in the following theorem we give a summary of the most important properties which are determined by the isomorphism type of the universal enveloping algebra.

Theorem ([27]). Let $L$ and $H$ be Lie algebras and $\alpha: U(L) \rightarrow U(H)$ an algebra isomomorphim, and suppose that $L$ is finite-dimensional. Then the following hold.

1. $\operatorname{dim} L=\operatorname{dim} H$ and $\operatorname{dim} L / L^{\prime}=\operatorname{dim} H / H^{\prime}$.

2. If $L$ is nilpotent, then so is $H$. Moreover, in this case the nilpotency classes of $L$ and $H$ coincide.

3. $L^{\prime} / L^{\prime \prime} \cong H^{\prime} / H^{\prime \prime}$; in particular, $\operatorname{dim} L^{\prime} / L^{\prime \prime}=\operatorname{dim} H^{\prime} / H^{\prime \prime}$.

4. If $L$ is metabelian, then so is $H$.

5. If $L$ is solvable, then so is $H$.

6. If $M$ and $N$ are ideals of $L$ and $H$, respectively; such that $\alpha(M U(L))=N U(H)$. Then, $M / M^{\prime} \cong N / N^{\prime}$.

The properties that are determined by the isomorphism type of the universal enveloping algebra play an important role in the study of the isomorphism problem. More precisely, one way to find more classes of Lie algebras in which the isomorphism problem has a positive solution, is to find properties that are determined by the isomorphism type of the universal enveloping algebra and to analyze these properties to define such classes. For example, Usefi [27, Proposition 5.2] showed that the isomorphism type of $L / \gamma_{3}(L)$ is determined by the isomorphism type of $U(L)$. Thus, if $\mathcal{C}$ is the class of nilpotent Lie algebras of nilpotency class two (i.e $\gamma_{3}(L)=0$ ), we have that the isomorphism problem has a positive solution in $\mathcal{C}$. One of the objectives of this thesis is to find some properties that are determined by the isomorphism type of the universal enveloping algebras, and then to find some classes of the Lie algebras in which the isomorphism problem has a positive solution.

Our main contribution to the isomorphism problem is to prove the following theorem. 
Theorem 3.2.1 (Main Theorem) Let $L, H$ be solvable Lie algebras of dimension 4 over a field of characteristic 0 . If $U(L) \cong U(H)$, then $L \cong H$.

The proof of Theorem 3.2.1 basically consists of two steps. First, using the classification by de Graaf we divide the Lie algebras into five groups (this division can be found in Section 3.1) in a way that two Lie algebras in different groups cannot have isomorphic universal enveloping algebras. Second, we analyze each group in order to ensure that in the same group do not exist two non-isomorphic Lie algebras with isomorphic universal enveloping algebras. This is a crucial (and more complicated) part of the proof. The second step of the proof of Theorem 3.2.1 is scattered in several parts in Chapters 4,5 and 6. In each of the chapters we develop several techniques that help us in the second step of the proof of Theorem 3.2.1.

This theorem is the most important result of this thesis and all results here were motivated to try to find a proof of this theorem. However, not all of these results have a direct relationship with Theorem 3.2.1, but we believe that all of them were important to better understand the isomorphism problem and some issues that are related to it. We summarise our principal results in the next two sections.

\subsection{Center, semicenter and Frobenius semiradical}

The first sections of Chapter 4 are dedicated to the study of the center and to a result which helps us to give counterexamples for the isomorphism problem. This result was inspired by the analysis of several examples of the paper by Usefi and Schneider [29]. In the following theorem we put together Theorem 4.2.1 and Corollary 4.2 .2 , which are the two principal results in this direction. Given a Lie algebra $L$ the set of primitive elements of $U(L)$ is denoted by $\mathcal{P}(U(L))$, see Section 2.4. The direct and semidirect product of Lie algebras is defined before Lemma 2.1.3.

Theorem. Let $L=M \oplus\langle x\rangle$ and $H=N \rtimes\langle y\rangle$ be two finite-dimensional nilpotent Lie algebras, where $M$ and $N$ are ideals of $L$ and $H$, respectively. Suppose that $M \cong N$ and $\operatorname{gr}(L) \cong \operatorname{gr}(H)$, and there exists an element $u \in U(N)$ such that $\left(\operatorname{ad}_{U(H)} u\right)(N) \subseteq$ $N$ and $\left.\operatorname{ad}_{U(H)} u\right|_{N}=\left.\operatorname{ad}_{U(H)} y\right|_{N}$. Then, there exists an isomorphsim between $U(L)$ and $U(H)$. Moreover, if $u \in \mathcal{P}(U(N))$ then $U(L) \cong U(H)$ as bialgebras. 
In Chapter 4 we see how this theorem can be used to produce counterexamples for the isomorphism problem in prime characteristic. At the moment, we are unable to produce a pair of Lie algebras that satisfies the conditions of the theorem above in characteristic zero. In fact, in characteristic zero the isomorphism problem is still an open problem in general.

In Chapter 4 we also defined the semicenter $S z(U(L))$ of $U(L)$, see Section 4.3 . Another important fact shown in Chapter 4 is Theorem 4.3.3, which basically says that $S z(U(L))$ is invariant under isomorphisms between universal enveloping algebras.

We listed in Section 1.1 several invariants of Lie algebras that are determined by the isomorphism type of the universal enveloping algebra. It is interesting to note that the dimension of the center does not appear among these invariants. In fact, examples show that if char $\mathbb{F}$ is a prime then there exist Lie algebras $L$ and $H$ such that $U(L) \cong U(H)$, but $\operatorname{dim} Z(L) \neq \operatorname{dim} Z(H)$ (see Example 2.1.6). On the other hand, no such examples exist in characteristic zero and it is conjectured that in characteristic zero the dimension of the center of $L$ is determined by the isomorphism type of $U(L)$. Inspired by this conjecture, in Section 4.4 we study the Frobenius semiradical $F(L)$ of a Lie algebra $L$, see Section 4.4 for the definition of $F(L)$. This object was introduced by Ooms in [21] and it is very important to understand the structure of $U(L)$. In particular, we study the square integrable Lie algebras, which by definition are the Lie algebras $L$ such that $F(L)=Z(L)$. More precisely, we shall show the following theorems.

Theorem 4.4.6 Suppose that $L$ is finite-dimensional square integrable Lie algebra over a field $\mathbb{F}$ of characteristic 0. Then $U(Z(L))=Z(U(L))$.

Theorem 4.4.8 Suppose that $L$ and $H$ are finite-dimensional square integrable Lie algebras over a field $\mathbb{F}$ of characteristic 0 , and that $\varphi: U(L) \rightarrow U(H)$ is an algebra isomorphism. Then, $\operatorname{dim} Z(L)=\operatorname{dim} Z(H)$.

Another important result in Chapter 4 is Theorem 4.4.10. This theorem describes all solvable Lie algebras of dimension 4 that are square integrable. For the proof of Theorem 4.4.10 we use the classification given by de Graaf [5]. As a direct consequence of Theorem 4.4.10, we calculate the center of the universal enveloping algebras for square integrable solvable 4-dimensional Lie algebra over a field of characteristic 0 . Studying the center of universal enveloping algebras is of independent interest. In 
fact, the center of $U(L)$ plays an important role in the representation theory of $L$; see for example [8, Chapter 4] and [30, Section 5.1].

\subsection{Structure of $U(L)$ for certain Lie algebras}

In Section 5.1 we present a construction. For a Lie algebra $L$, an abelian ideal $M$ of $L$ and a maximal two-sided ideal $\mathfrak{m}$ of $U(L)$, such that $U(L) / \mathfrak{m} \cong \mathbb{F}$, outputs another Lie algebra $L_{M}$ which is invariant under a certain group of automorphisms of $U(L)$.

One of the most important results in Section 5.1 is Theorem 5.1.4, which provides a condition for the isomorphism type of $L_{M}$ to be determined by the isomorphism type of $U(L)$. Consequently, we have that the Lie algebra $L_{M}$ is invariant under a certain group of automorphisms of $U(L)$.

Theorem 5.1.4 Let $L$ and $H$ be finite-dimensional Lie algebras over an arbitrary field $\mathbb{F}$ and let $M$ and $N$ be abelian ideals of $L$ and $H$, respectively. Suppose that $\alpha: U(L) \rightarrow U(H)$ is an algebra isomorphism such that $\alpha(M U(L))=N U(H)$. Let $\mathfrak{m}$ be a maximal (two-sided) ideal of $U(L)$ such that $U(L) / \mathfrak{m} \cong \mathbb{F}$ and $M \subseteq \mathfrak{m}$ and set $\mathfrak{n}=\alpha(\mathfrak{m})$. Then $\alpha$ induces an isomorphism between the Lie algebras $L_{M}$ and $H_{N}$.

Another important result of Chapter 5 is Proposition 5.2.1, which is a partial result towards the second step of the proof of Theorem 3.2.1.

In Chapter 6, we study the structure of the universal enveloping algebra $U(L)$ for Lie algebras of the form $L=M \rtimes\langle x\rangle$, where $M$ is an ideal. These Lie algebras have some advantages. First, the complement (as vector spaces) of $M$ is a Lie algebra; in general this is not true if $\operatorname{dim}(L / M) \geqslant 2$. Second, $U(L / M)$ can be viewed as the polynomial ring $\mathbb{F}[x]$; and the automorphisms of $\mathbb{F}[x]$ are well understood. We will exploit this.

One of most important results in Chapter 6 is Proposition 6.1.1 and its immediate corollary, which we state next. These were inspired by a corresponding result for group rings originally proved by Whitcomb in [33], see also [24, Theorem 9.3.13]. With this result Whitcomb showed that the isomorphism problem for integral group rings has a positive solution for metabelian groups. 
Proposition 6.1.1 Let $L=M \rtimes\langle x\rangle$ and $H=N \rtimes\langle y\rangle$ be finite-dimensional Lie algebras over an arbitrary field, where $M$ and $N$ are ideals of $L$ and $H$, respectively. Suppose that $\alpha: U(L) \rightarrow U(H)$ is an algebra isomorphism such that $\alpha(M U(L))=$ $N U(H)$. Then, $L / M^{\prime} \cong H / N^{\prime}$.

Corollary 6.1.2 Suppose that $L, H, N, M$ are as in Proposition 6.1.1 and that $\alpha$ : $U(L) \rightarrow U(H)$ is an algebra isomorphism. Then the following are valid.

1. If $\alpha(M U(L))=N U(H)$ and either $M$ or $N$ is abelian, then $L \cong H$.

2. If $M=L^{\prime}$ and $N=H^{\prime}$, then $L / L^{\prime \prime} \cong H / H^{\prime \prime}$. In particular; if either $L$ or $H$ is metabelian, then $L \cong H$.

As stated in Section 1.1, one of the objectives of this thesis is to find the properties of a Lie algebra $L$ which are determined by the isomorphism type of $U(L)$, and to use these properties to define a class in which the isomorphism problem has a positive solution. Let us see what we can do with item (2) of Corollary 6.1.2. In fact, from Corollary 6.1.2 (2) we have that the isomorphism type of $L / L^{\prime \prime}$ is determined by the isomorphism type of $U(L)$. Thus, if $\mathcal{C}$ is the class of finite-dimensional metabelian Lie algebras with derived subalgebra of codimension 1, then the isomorphism problem has a positive solution in $\mathcal{C}$.

Another result of Chapter 6 that is worth mentioning is Proposition 6.1.4, which we state next. This is important because it is used in a proposition which is a partial result towards the second step of the proof of Theorem 3.2.1.

Proposition 6.1.4 Suposse that $L$ and $H$ are two finite-dimensional metabelian Lie algebras over a field $\mathbb{F}$ of characteristic 0 , and that $L=\left(L^{\prime}+Z(L)\right) \rtimes\langle x\rangle$ and $H=\left(H^{\prime}+Z(H)\right) \rtimes\langle y\rangle$. If $\alpha: U(L) \rightarrow U(H)$ is an algebra isomorphism, then $L \cong H$.

In the proof of this proposition, the hypothesis that characteristic of the field is zero is necessary, since we use a result proved by Ooms [21], which is valid only in this case.

In Chapter 6, we also present other propositions which are used in the second step of the proof of Theorem 3.2.1 and the end of the proof of Theorem 3.2.1. This final step consists of putting together the partial results shown in the previous chapters. 


\section{Chapter 2}

\section{Background}

This chapter is dedicated to establish the bases of this thesis. We will not systematically develop the topics raised in this chapter, as they have already been dealt with extensively elsewhere, but we will give references to everything that is not proved. The main objective is to present the tools that will serve for a systematic development of this thesis.

This chapter is divided into four sections. In Section 2.1, we present the concepts of Lie algebras and their universal enveloping algebras. These concepts are really very basic and one can find a lot of information about them in the classical books on Lie algebras, for example [2,11,13]. Section 2.2 is devoted to introduce the concept of ring of quotients of the universal enveloping algebra, for this section we refer the Dixmier's book [8]. In Section 2.3, we will see some properties of Lie algebras that are preserved by an isomorphism between their universal enveloping algebras. The main reference for this section is the paper by Riley and Usefi [27]. Section 2.4 is devoted to present the definition of Hopf algebras and to introduce the Hopf algebra structure of universal enveloping algebras. References for this section are Sweedler's classic book 31 and the paper by Milnor and Moore [17].

\subsection{Lie algebras and their universal enveloping al- gebras}

Regardless of the context, the notation $\mathbb{F}$ is reserved for a field. We assume that $\mathbb{F}$ has an arbitrary characteristic, unless otherwise stated. 
Definition 2.1.1. A vector space $L$ over a field $\mathbb{F}$ endowed with a bilinear map $[\cdot, \cdot]: L \times L \rightarrow L$ is called a Lie algebra if

1. $[x, x]=0$ for all $x \in L$,

2. $[x,[y, z]]+[y,[z, x]]+[z,[x, y]]=0$ for all $x, y, z \in L$.

The operation [., $\cdot]$ in $L$ is called the bracket of $L$ and the second condition is called the Jacobi identity. A subspace $K$ of $L$ is called a (Lie) subalgebra if $[K, K] \subseteq K$. If $H$ is another Lie algebra, then a linear map $f: L \rightarrow H$ is a (Lie) homomorphism if $f([x, y])=[f(x), f(y)]$ for all $x, y \in L$. A derivation on $L$ is a linear map $D: L \rightarrow L$ such that $D([x, y])=[D(x), y]+[x, D(y)]$ for all $x, y \in L$.

\section{Example 2.1.1.}

1. If $A$ is an associative algebra, then $A$ is a Lie algebra with the bracket defined by $[x, y]=x y-y x$ for all $x, y \in A$.

2. If $V$ is a vector space of dimension $n$, then $\operatorname{End}(V)$ is an associative algebra. By item $1, \operatorname{End}(V)$ is a Lie algebra. This algebra is denoted by $\mathfrak{g l}(V)\left(\right.$ or $\left.\mathfrak{g l}_{n}(\mathbb{F})\right)$ and is called the general linear Lie algebra.

3. The trace $\operatorname{tr}(x)$ of an endomorphism $x \in \mathfrak{g l}(V)$ for a finite-dimensional vector space $V$ can be defined as the trace of any matrix representing $x$. The set $\mathfrak{s l}(V)=\{x \in \mathfrak{g l}(V) \mid \operatorname{tr}(x)=0\}$ is a Lie subalgebra of $\mathfrak{g l}(V)$, and is called the special linear Lie algebra.

4. The set $\operatorname{Der}(L)=\{D \in \mathfrak{g l}(L) \mid D$ is a derivation of $L\}$ of derivations of a Lie algebra $L$ is itself a Lie algebra.

Given $x \in L$ we can define ad $x: L \rightarrow L$, by ad $x(y)=[x, y]$, which is a derivation of $L$. The map ad $: L \rightarrow \operatorname{Der}(L)$, sending $x$ to ad $x$, is called the adjoint representation of $L$.

If $A$ is an associative algebra and $x, y \in A$, then

$$
(\operatorname{ad} x)^{m}(y)=\sum_{j=0}^{m}(-1)^{m-j}\left(\begin{array}{c}
m \\
j
\end{array}\right) x^{j} y x^{m-j},
$$


see [30, Proposition 1.3]. If char $\mathbb{F}=p$, then $p \mid\left(\begin{array}{l}p \\ j\end{array}\right)$ for every $1 \leqslant j \leqslant p-1$, and it follows that

$$
(\operatorname{ad} x)^{p}(y)=x^{p} y-y x^{p}=\operatorname{ad} x^{p}(y) .
$$

A subspace $K$ of $L$ is an ideal of $L$ if $[K, L] \subseteq K$ (or equivalently $[L, K] \subseteq K$ ). Obviously 0 and $L$ are ideals. Another less trivial example of an ideal is the center $Z(L)=\{x \in L \mid[x, y]=0$ for all $y \in L\}$ of a Lie algebra $L$. A Lie algebra $L$ is abelian if $Z(L)=L$. Another example of an ideal is the derived subalgebra of $L$, denoted by $L^{\prime}$ which is the subspace generated by the elements $[x, y]$ with $x, y \in L$. Note that $L$ is abelian if and only if $L^{\prime}=0$.

It is clear that ideals are kernels of homomorphisms of Lie algebras. With this in mind we have that $\mathfrak{s l}(V)$ is an ideal of $\mathfrak{g l}(V)$, since $\mathfrak{s l}(V)$ is the kernel of the homomorphism $\operatorname{tr}: \mathfrak{g l}(V) \rightarrow \mathbb{F}$, sending $x$ to $\operatorname{tr}(x)$. Given an ideal $K$ and a subalgebra $M$ of $L$, the $\operatorname{map} \operatorname{ad}_{M, K}: M \rightarrow \mathfrak{g l}(K)$, seding $x$ to ad $\left.x\right|_{K}$, is called adjoint representation of $M$ on $K$. The centralizer of a subset $S$ of $L$ is defined by the set $C_{L}(S)=\{x \in L \mid[x, s]=0$ for every $s \in S\}$. From the Jacobi identity it follows that $C_{L}(S)$ is a subalgebra of $L$. Moreover, if $S$ is an ideal of $L$, then so is $C_{L}(S)$. It is easy to verify that the kernel of $\operatorname{ad}_{M, K}$ is $C_{L}(K) \cap M$.

The lower central series of $L$ is defined inductively by $\gamma_{1}(L)=L$ and $\gamma_{n}(L)=$ $\left[\gamma_{n-1}(L), L\right]$ for $n \geqslant 2$. The second term coincides with $L^{\prime}$. Note that $\gamma_{n}(L)$ is an ideal of $L$ for all $n \geqslant 1$. A Lie algebra $L$ is called nilpotent if there exists a positive integer $n$ such that $\gamma_{n}(L)=0$. The minimal number $c$ such that $\gamma_{c+1}(L)=0$ is called the nilpotency class of $L$. The graded Lie algebra of $L$ is defined by

$$
\operatorname{gr}(L)=\bigoplus_{i \geqslant 1} \gamma_{i}(L) / \gamma_{i+1}(L)
$$

where the bracket in $\operatorname{gr}(L)$ is given by

$$
\left[x_{i}+\gamma_{i+1}(L), y_{j}+\gamma_{j+1}(L)\right]=\left[x_{i}, y_{j}\right]+\gamma_{i+j+1}(L)
$$

for all $x_{i} \in \gamma_{i}(L)$ and $y_{j} \in \gamma_{j}(L)$. A calculation shows that

$$
\gamma_{n}(\operatorname{gr}(L))=\bigoplus_{i \geqslant n} \gamma_{i}(L) / \gamma_{i+1}(L)
$$


Thus, if $L$ is nilpotent, then so is $\operatorname{gr}(L)$. Moreover, the nilpotency class of $L$ and $\operatorname{gr}(L)$ coincide. We also have that $\gamma_{i}(L) / \gamma_{i+1}(L) \cong \gamma_{i}(\operatorname{gr}(L)) / \gamma_{i+1}(\operatorname{gr}(L))$.

We say that $L$ is residually nilpotent if $\bigcap_{n \geqslant 1} \gamma_{n}(L)=0$. It is clear that every nilpotent Lie algebra is residually nilpotent. However the converse is false, as we will see this in Example 2.1.2. For each $y$ we define the height of $y$ as $\nu(y)=\max \{n \mid$ $\left.y \in \gamma_{n}(L)\right\}$ and infinite when this maximum does not exist. We shall call a basis $\left\{x_{1}, x_{2}, \ldots\right\}$ of $L$ homogeneous if each $\gamma_{n}(L)$ is generated as a vector space by the set $\left\{x_{i} \mid \nu\left(x_{i}\right) \geqslant n\right\}$.

\section{Example 2.1.2.}

1. Let $L$ be the Lie algebra with basis $\left\{x_{1}, x_{2}, \ldots\right\}$, and non-zero brackets $\left[x_{i}, x_{1}\right]=$ $x_{i+1}$ for $i \geqslant 2$. We have that $\gamma_{n}(L)=\left\langle x_{n+1}, x_{n+2}, \ldots\right\rangle$ for $n \geqslant 2$. Thus, $L$ is not nilpotent. We claim that $L$ is residually nilpotent. In fact, let $x \in \bigcap_{n \geqslant 1} \gamma_{n}(L)$, then this is written uniquely as $x=\alpha_{i_{1}} x_{i_{1}}+\cdots+\alpha_{i_{k}} x_{i_{k}}$. Since $x \in \gamma_{i_{k}+1}(L)$, we can write this as $x=\alpha_{j_{1}} x_{j_{1}}+\cdots+\alpha_{j_{r}} x_{j_{r}}$ with $j_{l} \geqslant i_{k}+2$ for $1 \leqslant l \leqslant r$. But since the representation of $x$ as linear combination of elements of a basis of $L$ is unique, we have that the coefficients of $x$ in both expressions are equal to 0 . Thus $\bigcap_{n \geqslant 1} \gamma_{n}(L)=0$.

2. Let $L$ be a Lie algebra, suppose that there exists $n$ such that for every $m \geqslant n$ we have that $\gamma_{m}(L)=\gamma_{n}(L)$. Then there exist a homogeneous basis for $L$. In fact, we can complete a basis of $\gamma_{n}(L)$ to a basis of $\gamma_{n-1}(L)$ and this basis of $\gamma_{n-1}(L)$ to a basis of $\gamma_{n-2}(L)$ and so on until a basis of $L$. This basis obtained is a homogeneous basis of $L$.

The derived series of $L$ is defined by $\delta_{0}(L)=L$ and $\delta_{n}(L)=\left[\delta_{n-1}(L), \delta_{n-1}(L)\right]$ for $n \geqslant 1$. Note that $\delta_{1}(L)=L^{\prime}$. The term $\delta_{2}(L)$ is also denoted by $L^{\prime \prime}$. A Lie algebra $L$ is called solvable if there exists a positive integer $n$ such that $\delta_{n}(L)=0$. It is clear that every nilpotent Lie algebra is solvable. The minimal number $l$ such that $\delta_{l}(L)=0$ is called the derived length of $L$. A Lie algebra $L$ is said to be metabelian if $\delta_{2}(L)=0$.

Given two Lie algebras $L, H$ and a Lie homomorphism $\phi: L \rightarrow \operatorname{Der}(H)$ we can construct another Lie algebra considering the vector space $H \oplus L$ with the bracket

$$
[(a, x),(b, y)]=(\phi(x) b-\phi(y) a+[a, b],[x, y])
$$


for $a, b \in H$ and $x, y \in L$. This algebra is called the semidirect product of $H$ and $L$, and is denoted by $H \rtimes_{\phi} L$. The map $H \rtimes_{\phi} L \rightarrow H \rtimes_{\phi} L$ defined by $(a, x) \mapsto(0, x)$ is a Lie homomorphism with kernel $H \oplus 0$ and image $0 \oplus L$. Thus $L$ and $H$ can be viewed as a subalgebra and as an ideal of $H \rtimes_{\phi} L$, respectively.

Let $I$ be an ideal and let $K$ be a subalgebra of a Lie algebra $L$ such that $L=$ $I \oplus K$ as vector spaces. Then we have $L=I \rtimes_{\phi} K$, where $\phi=\operatorname{ad}_{K, I}$ is the adjoint representation of $K$ on $I$. If $L=I \rtimes_{\phi} K$ and $\phi=\operatorname{ad}_{K, I}$ is the trivial action, then $I$ and $K$ are ideals and we say that $L$ is the direct product of $I$ and $K$, and we write $L=I \oplus K$.

Lemma 2.1.3 ([2], Proposition 7). Let $H_{1} \rtimes_{\phi_{1}} L_{1}$ and $H_{2} \rtimes_{\phi_{2}} L_{2}$ be two Lie algebras and let $\psi_{1}: H_{1} \rightarrow H_{2}$ and $\psi_{2}: L_{1} \rightarrow L_{2}$ be Lie homomorphism such that the diagram

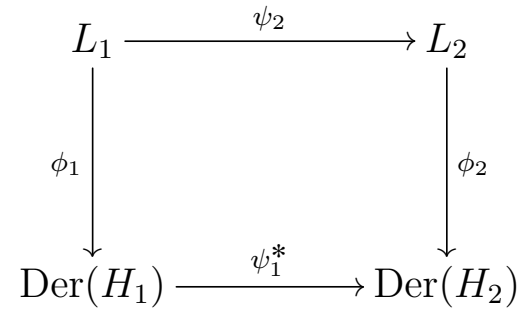

is commutative, where $\psi_{1}^{*}(\delta)=\psi_{1} \delta \psi_{1}^{-1}$ is the map induced by $\psi_{1}$. Then the map defined by $(a, x) \mapsto\left(\psi_{1}(a), \psi_{2}(x)\right)$ is a Lie homomorphism between $H_{1} \rtimes_{\phi_{1}} L_{1}$ and $H_{2} \rtimes_{\phi_{2}} L_{2}$.

Note that the commutativity of the diagram above is equivalent to the compatibility of the actions defined by $\phi_{1}$ and $\phi_{2}$.

Let $V$ be a vector space over $\mathbb{F}$. Suppose that $\left\{x_{1}, x_{2}, \ldots\right\}$ is a basis of $V$. Then the free associative algebra generated by $V$ is denoted by $\mathbb{F}\left\langle x_{1}, x_{2}, \ldots\right\rangle$. The elements of $\mathbb{F}\left\langle x_{1}, x_{2}, \ldots\right\rangle$ are polynomials in the non-commuting variables $\left\{x_{1}, x_{2}, \ldots\right\}$ with coefficient from $\mathbb{F}$. Here, the coefficients are supposed to commute with each $x_{i}$. It is clear that the linear map $i: V \rightarrow \mathbb{F}\left\langle x_{1}, x_{2}, \ldots\right\rangle$, defined by $x_{i} \mapsto x_{i}$, is injective. Suppose that $B$ is an associative algebra and that $f: V \rightarrow B$ is a linear map. We can define an algebra homomorphism $\tilde{f}: \mathbb{F}\left\langle x_{1}, x_{2}, \ldots\right\rangle \rightarrow B$, sending each monomial $x_{i_{1}} \cdots x_{i_{k}}$ to $f\left(x_{i_{1}}\right) \cdots f\left(x_{i_{k}}\right)$ and extending it linearly to $\mathbb{F}\left\langle x_{1}, x_{2}, \ldots\right\rangle$. It follows immediately that $\tilde{f} \circ i=f$. The algebra homomorphism $\tilde{f}$ is unique such that $\tilde{f} \circ i=f$. 
Definition 2.1.2. Let $L$ be a Lie algebra over a field $\mathbb{F}$. Suppose that $\mathcal{B}=\left\{x_{1}, x_{2}, \ldots\right\}$ is a basis of $L$. The universal enveloping algebra $U(L)$ of $L$ is defined as the associative algebra

$$
U(L):=\frac{\mathbb{F}\left\langle x_{1}, x_{2}, \ldots\right\rangle}{\left\langle x_{i} x_{j}-x_{j} x_{i}-\left[x_{i}, x_{j}\right]\right\rangle},
$$

where $\left\langle x_{i} x_{j}-x_{j} x_{i}-\left[x_{i}, x_{j}\right]\right\rangle$ is a two-sided ideal of $\mathbb{F}\left\langle x_{1}, x_{2}, \ldots\right\rangle$ generated by the elements $x_{i} x_{j}-x_{j} x_{i}-\left[x_{i}, x_{j}\right]$, for al $x_{i}, x_{j} \in \mathcal{B}$. In this last expression, $\left[x_{i}, x_{j}\right]$ denotes the Lie bracket in $L$.

We have a natural projection $\pi: \mathbb{F}\left\langle x_{1}, x_{2}, \ldots\right\rangle \rightarrow U(L)$. The image of $x_{i}$ via the algebra homomorphism $\pi$ will be denoted by the same $x_{i}$. Thus, the image of an element in $\mathbb{F}\left\langle x_{1}, x_{2}, \ldots\right\rangle$ will be denoted by the same symbol. To multiply elements of $U(L)$ we use the relations $x_{i} x_{j}-x_{j} x_{i}-\left[x_{i}, x_{j}\right]=0$.

Composing the natural projection $\pi: \mathbb{F}\left\langle x_{1}, x_{2}, \ldots\right\rangle \rightarrow U(L)$ with the linear map $i: L \rightarrow \mathbb{F}\left\langle x_{1}, x_{2}, \ldots\right\rangle$, defined before Definition 2.1.2, we obtain a Lie homomorphism $\iota: L \rightarrow U(L)$. Now, suppose that $B$ is another associative algebra and that $f: L \rightarrow B$ is a Lie homomorphism (in particular $f$ is a linear map). Therefore, as we saw before Definition 2.1.2, there exists a unique algebra homomorphism $\tilde{f}$ such that $\tilde{f} \circ i=f$. Thus, the lower triangle in the following diagram is commutative:

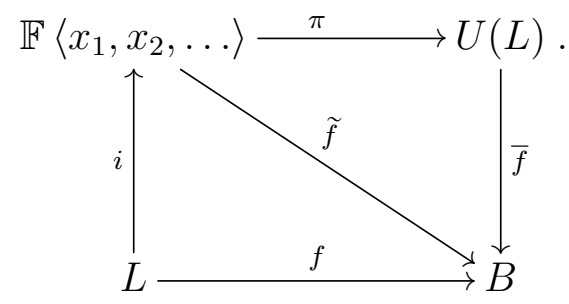

Since $f$ is a Lie homomorphism we have that $\left\langle x_{i} x_{j}-x_{j} x_{i}-\left[x_{i}, x_{j}\right]\right\rangle \subseteq \operatorname{ker} \tilde{f}$, and this implies the existence of an algebra homomorphism $\bar{f}: U(L) \rightarrow B$ such that the upper triangle is commutative. Hence $\bar{f} \circ \iota=\bar{f} \circ \pi \circ i=\tilde{f} \circ i=f$. The uniqueness of $\tilde{f}$ in combination with the surjectivity of $\pi$ implies the uniqueness of $\bar{f}$. Therefore, we have shown the following lemma.

Lemma 2.1.4. The pair $(\iota, U(L))$ satisfies the following universal property: given an associative algebra $B$ and and Lie homomorphism $f: L \rightarrow B$, there exists a unique 
algebra homomorphism $\bar{f}: U(L) \rightarrow B$ such that $\bar{f} \circ \iota=f$; that is, the following diagram is commutative:

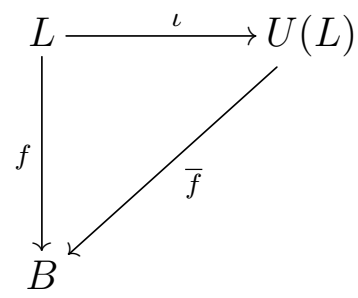

Moreover, if $U$ is an associative algebra and $j: L \rightarrow U$ is such that the pair $(j, U)$ satisfies the universal property above then $U \cong U(L)$.

Before we start with some examples, we need to make an observation about the notation that will be widely used. To describe a Lie algebra $L$ we display a basis $\left\{x_{1}, x_{2} \ldots\right\}$ of $L$ and the non-zero brackets $\left[x_{i}, x_{j}\right]$ of elements of this basis. For instance, let $L=\langle x, y, z \mid[x, y]=z\rangle$. Notice that $[x, z]$ and $[y, z]$ do not appear explicitly in the presentation above, since $[x, z]=[y, z]=0$.

\section{Example 2.1.5.}

1. If $L=0$, then $U(L)=\mathbb{F}$.

2. If $L=\left\langle x_{1}, x_{2}, \ldots\right\rangle$ is an abelian Lie algebra (possibly infinite-dimensional) then $U(L)$ is commutative. In fact, we have that

$$
U(L)=\frac{\mathbb{F}\left\langle x_{1}, x_{2}, \ldots\right\rangle}{\left\langle x_{i} x_{j}-x_{j} x_{i}\right\rangle} \cong \mathbb{F}\left[x_{1}, x_{2}, \ldots\right]
$$

is a polynomial ring.

3. Let $L=\langle x, y \mid[x, y]=y\rangle$. Then

$$
U(L)=\frac{\mathbb{F}\langle x, y\rangle}{\langle x y-y x-y\rangle} .
$$

Thus, $U(L)$ is isomorphic to the skew polynomial ring $\mathbb{F}[x][y ; \sigma]$, where $\sigma$ is the automorphism of $\mathbb{F}[x]$ sending $x$ to $x-1$, see $[14$, Example (1.7)]. In this ring we have $y x=\sigma(x) y=(x-1) y$, and so $x y-y x-y=0$. It is also possible describe $U(L)$ as the differential polynomial ring $\mathbb{F}[y][x, \delta]$, where $\delta$ is the derivation of $\mathbb{F}[y]$ defined by $\delta(f)=y \frac{d}{d y}(f)$, see $[14$, Example (1.9)]. In this ring we have that $x y=y x+\delta(y)=y x+y$, and so $x y-y x-y=0$. 
4. Let $H=\left\langle x_{1}, \ldots, x_{n}, y_{1}, \ldots, y_{n}, z\right|\left[x_{i}, y_{i}\right]=z$ for $\left.1 \leqslant i \leqslant n\right\rangle$ be the $(2 n+1)$ dimensional Heisenberg Lie algebra. Thus

$$
U(H)=\frac{\mathbb{F}\left\langle x_{1}, \ldots, x_{n}, y_{1}, \ldots, y_{n}, z\right\rangle}{I},
$$

where $I$ is the two-sided ideal generated by the elements

$$
x_{i} y_{i}-y_{i} x_{i}-z \quad \text { for } 1 \leqslant i \leqslant n,
$$

and

$$
x_{i} x_{j}-x_{j} x_{i}, \quad y_{i} y_{j}-y_{j} y_{i}, \quad x_{i} y_{j}-y_{j} x_{i}, \quad \text { for } i \neq j \text {. }
$$

Let

$$
A_{n}(\mathbb{F})=\frac{\mathbb{F}\left\langle x_{1}, \ldots, x_{n}, y_{1}, \ldots, y_{n}\right\rangle}{\widetilde{I}}
$$

be the $n$-th Weyl algebra. Here $\widetilde{I}$ denotes the two-sided ideal generated by the elements

$$
x_{i} y_{i}-y_{i} x_{i}-1 \quad \text { for } 1 \leqslant i \leqslant n,
$$

and

$$
x_{i} x_{j}-x_{j} x_{i}, \quad y_{i} y_{j}-y_{j} y_{i}, \quad x_{i} y_{j}-y_{j} x_{i}, \quad \text { for } i \neq j
$$

see [14, Example (1.3)]. Then, the map defined by $x_{i} \mapsto x_{i}, y_{i} \mapsto y_{i}, z \mapsto 1$ for all $1 \leqslant i \leqslant n$, is an algebra homomorphism of $U(H)$ onto $A_{n}(\mathbb{F})$. The kernel of this map is the ideal generated by $z-1$, thus

$$
\frac{U(H)}{\langle z-1\rangle} \cong A_{n}(\mathbb{F})
$$

For more details of the terminology introduced in this example we refer to Chapter 1 of $[14$.

With the definitions provided until now, we can state the problem that motivates this thesis.

The isomorphism problem for universal enveloping algebras of Lie algebras: If $L, H$ are Lie algebras, does $U(L) \cong U(H)$ imply that $L \cong H$ ?

We give a counterexample in characteristic 2, counterexamples in arbitrary characteristic can be found in Example 4.2.3. 
Example 2.1.6. Let $\mathbb{F}$ be a field with char $\mathbb{F}=2$. We consider two Lie algebras of dimension 5 over $\mathbb{F}$

$$
\begin{aligned}
& L=\left\langle x_{1}, x_{2}, x_{3}, x_{4}, x_{5} \mid\left[x_{1}, x_{2}\right]=x_{4},\left[x_{1}, x_{4}\right]=x_{5}\right\rangle \\
& H=\left\langle x_{1}, x_{2}, x_{3}, x_{4}, x_{5} \mid\left[x_{1}, x_{2}\right]=x_{4},\left[x_{1}, x_{4}\right]=x_{5},\left[x_{2}, x_{3}\right]=x_{5}\right\rangle .
\end{aligned}
$$

It is proved in [29, Theorem 3.1] that the map induced by $x_{1} \mapsto x_{1}, x_{2} \mapsto x_{2}$, $x_{3} \mapsto x_{3}+x_{1}^{2}, x_{4} \mapsto x_{4}$ and $x_{5} \mapsto x_{5}$ is an isomorphism between $U(L)$ and $U(H)$ (also we verify this in Chapter 4$)$. However, since $Z(L)=\left\langle x_{3}, x_{5}\right\rangle$ and $Z(H)=\left\langle x_{5}\right\rangle$, it follows that $L \not H$.

As we see in the example above, the isomorphism problem in general, has a negative solution. However, there exist several classes of Lie algebras in which the isomorphism problem has a positive solution. For example, we will show that the isomorphism problem has a positive solution for finite-dimensional metabelian Lie algebras $L$ in which the codimension of $L^{\prime}$ is one, see Corollary 6.1.2 (2). One of the main objectives of this thesis is to study the isomorphism problem in some classes of Lie algebras, and obtain results that help us decide if the isomorphism problem is true or false in some cases.

Let $L$ be a Lie algebra with basis $\left\{x_{1}, x_{2}, \ldots\right\}$. The following question arises naturally: How to get a basis for $U(L)$ ? The answer for this question is given by the next theorem, which basically says that as a vector space $U(L)$ can be viewed as the polynomial ring over $\mathbb{F}$ with $\operatorname{dim} L$ indeterminates. This result is usually called the Poincaré-Birkhoff-Witt Theorem, see for example [1, Theorem 3.1]. A different, but equivalent version of the Poincaré-Birkhoff-Witt Theorem is presented in [11, Theorem p. 92]. A short and elegant proof of this theorem can be found in [1].

Theorem 2.1.7 (Poincaré-Birkhoff-Witt). If $\left\{x_{1}, x_{2}, \ldots\right\}$ is a basis for the Lie algebra $L$, then the monomials of the form

$$
x_{i_{1}}^{a_{1}} \cdots x_{i_{n}}^{a_{n}}
$$

where $n \geqslant 0, i_{1}<\cdots<i_{n}$ and $a_{i}>0$ for all $i$, form a basis for $U(L)$. Consequently, the map $\iota: L \rightarrow U(L)$ is injective. For $n=0$ the monomial in (2.2) is empty, and is identified with the identity element of $U(L)$. 
As is common in the literature, we refer to this theorem as the PBW Theorem. A monomial of the form 2.2 is said to be ordered. It is a consequence of the PBW Theorem that $L$ can be identified with a Lie subalgebra of $U(L)$. With this identification, given $x, y \in L$ we have that $y x=x y-[x, y]$.

Example 2.1.8. Let $L=\langle x, y \mid[x, y]=y\rangle$, with basis $\{x, y\}$. Consider the element $x y x$ in $U(L)$. This monomial is not ordered, since $x$ appears after of $y$, but we can reorder it using $y x=x y-[x, y]=x y-y$. In fact,

$$
\begin{aligned}
x y x & =x(y x) \\
& =x(x y-y) \\
& =x^{2} y-x y .
\end{aligned}
$$

This latter expression is a linear combination of ordered monomials, because $y$ appears only after $x$ inside each of these monomials.

The following theorem is very important in the study of the structure of $U(L)$, and is used for proving the existence of the ring of quotients of $U(L)$. Before we state this theorem, we need to establish some definitions of the theory of non-commutative rings. Let $A$ be a ring. An element $x \in A$ is called a zero divisor if there exist non-zero elements $a, b \in A$ such that $x a=b x=0$. A ring $A$ is called a domain if it has no zero divisor different from 0 . We say that $A$ is noetherian if it satisfies the ascending chain condition on left and right ideals; that is, given any chain

$$
I_{1} \subseteq I_{2} \subseteq \ldots \subseteq I_{k} \subseteq \ldots
$$

of left (or right) ideals, there exists $n$ such that $I_{m}=I_{n}$ for all $m \geqslant n$.

Theorem 2.1.9 ([13], Theorem 6 p. 166). Let $L$ be a Lie algebra. Then the following hold.

1. $U(L)$ is a domain.

2. If $L$ is finite-dimensional, then $U(L)$ is noetherian. 
Let $L$ be a Lie algebra with basis $\left\{x_{1}, x_{2}, \ldots\right\}$. The algebra homomorphism $\varepsilon_{L}$ : $U(L) \rightarrow \mathbb{F}$ induced by the map $x_{i} \mapsto 0$, for all $i$, is called the augmentation map. We denote the kernel of $\varepsilon_{L}$ by $\omega(L)$, which is called the augmentation ideal of $L$. The augmentation ideal $\omega(L)$ is equal to the unique maximal ideal of $U(L)$ that contains $x_{i}$ for all $i$. We also have $\omega(L)=L U(L)=U(L) L$. By the PBW Theorem, the set of the elements 2.2 with $n \geqslant 1$ form a basis of $\omega(L)$. The $n$-fold product of $\omega(L)$ is an ideal of $U(L)$ which is denoted by $\omega^{n}(L)$. Denoting $U(L)$ by $\omega^{0}(L)$, it is clear that the sequence $\left(\omega^{n}(L)\right)_{n \geqslant 0}$ forms a filtration of $U(L)$. An ideal $I$ in an associative algebra is called residually nilpotent if $\bigcap_{n \geqslant 1} I^{n}=0$. The following result gives necessary and sufficient conditions for the augmentation ideal $\omega(L)$ to be residually nilpotent. Recall that we defined the height $\nu(y)$ of an element $y \in L$ and homogeneous basis before Example 2.1.2.

Lemma 2.1.10. Let $L$ be a Lie algebra. Then,

1. $L$ is residually nilpotent as Lie algebra if and only if $\omega(L)$ is residually nilpotent as an associative ideal.

2. If $\left\{x_{1}, x_{2}, \ldots\right\}$ is a homogeneous basis of $L$, then the ordered monomials $x_{i_{1}}^{a_{1}} \cdots x_{i_{s}}^{a_{s}}$ with the property that $\sum_{k=1}^{s} a_{k} \nu\left(x_{i_{k}}\right) \geqslant n$ form a basis for $\omega^{n}(L)$.

Proof. Item (1) is contained in [27, Corollary 3.5] and item (2) in [27, Theorem 3.1 $(1)]$.

Lemma 2.1.10 also was sowed by Riley and Shalev [26] for restricted Lie algebras. This lemma also appear for arbitrary Lie algebras in Riley's paper [28. In 27] Usefi observed that in [28] it is assumed that every Lie algebra admits homogeneous basis, but he gives a counterexample showing that this is not true (see [27, Example C]).

The following lemma generalizes an argument presented in [29, Theorem 3.1] which was used to show that a certain surjective algebra homomorphism between universal enveloping algebras is an isomorphism.

Lemma 2.1.11. Let $L, H$ be Lie algebras and let $\varphi: U(L) \rightarrow U(H)$ be a surjective algebra homomorphism. Suppose that there exists a residually nilpotent ideal $I_{1}$ of $U(L)$ and an ideal $I_{2}$ of $U(H)$, such that $\varphi\left(I_{1}\right) \subseteq I_{2}$ and $U(L) / I_{1}^{k}$ is finite-dimensional and $\operatorname{dim} U(L) / I_{1}^{k}=\operatorname{dim} U(H) / I_{2}^{k}$ for every $k \geqslant 1$. Then $\varphi$ is an isomorphism. 
Proof. Since $\varphi\left(I_{1}\right) \subseteq I_{2}$ it follows that $\varphi\left(I_{1}^{k}\right) \subseteq I_{2}^{k}$ for $k \geqslant 1$. Thus, $\varphi$ induces surjective homomorphisms $\varphi_{k}$ between $U(L) / I_{1}^{k}$ and $U(H) / I_{2}^{k}$ for all $k \geqslant 1$, defined by $\varphi_{k}\left(w+I_{1}^{k}\right)=\varphi(w)+I_{2}^{k}$. Since $\varphi_{k}$ is a surjective linear transformation between finite-dimensional vector spaces of the same dimension, we have that $\varphi_{k}$ is injective for all $k \geqslant 1$. In order to show that $\varphi$ is injective let $z$ be a non-zero element in $U(L)$ such that $\varphi(z)=0$. By hypothesis $\bigcap_{k \geqslant 1} I_{1}^{k}=0$. This implies that there exists some $r \geqslant 1$ such that $z \notin I_{1}^{r}$. Since $\varphi_{r}$ is an isomorphism, we get $\varphi_{r}\left(z+I_{1}^{r}\right)=\varphi(z)+I_{2}^{r}=I_{2}^{r}$ is zero, which is a contradiction. Hence $\varphi$ is injective, and therefore an isomorphism between $U(L)$ and $U(H)$.

\subsection{Ring of quotients of the universal enveloping algebra}

In this section, we define a ring of quotients of certain rings and we use this to define the ring of quotients of a universal enveloping algebra. In the commutative case it is well-known that every domain has a quotient field, but this is not true in the non-commutative case. For this section we refer to Section 3.6 of Dixmier's book [8].

In this section $A$ denotes an arbitrary ring (with unity).

Definition 2.2.1. Let $S$ be a subset of $A$. We say that $S$ allows an arithmetic of fractions if the following condition are satisfied:

1. $1 \in S$;

2. the product of two elements of $S$ belongs to $S$;

3. the elements of $S$ are not zero divisors of $A$;

4. for $s \in S$ and $a \in A$ there exist $t \in S$ and $b \in A$ such that $a t=s b$;

5. for $s \in S$ and $a \in A$ there exist $t^{\prime} \in S$ and $b^{\prime} \in A$ such that $t^{\prime} a=b^{\prime} s$.

The items 4 and 5 in Definition 2.2.1 also are called Ore conditions, see 16, Chapter $2]$. In the commutative case Definition 2.2.1 defines the concept of multiplicative 
subsets. Given a set $S$ as above, we construct a $\operatorname{ring} B$ containing $A$ and such that every elements of $B$ can be written as $a s^{-1}$ or $t^{-1} b$, where $a, b \in A$ and $s, t \in S$. In the commutative case this is equivalent to the localization on $S$.

Let $S$ be a subset of $A$ allowing arithmetic of fractions. Let $(a, s),(b, t) \in A \times S$, and write $(a, s) \sim(b, t)$ if there exist $c, d \in A$ such that $a c=b d, s c=t d$ and $s c \in S$. The relation $\sim$ is an equivalence relation. Let $B$ be the set of equivalence classes of $A \times S$ under the relation $\sim$. We denote the equivalence class of $(a, s)$ by $a / s$. Let $a / s, b / t \in B$. Since $s \in A$ and $t \in S$, item 4 of Definition 2.2.1 implies that there exist $c \in A$ and $d \in S$ such that $s c=t d=e \in S$. Define

$$
(a / s)+(b / t)=(a c+b d) / e
$$

On other hand, since $b \in A$ and $s \in S$, item 4 of Definition 2.2.1 above implies that there exist $c \in A$ and $u \in S$ such that $b u=s c$. Define

$$
(a / s)(b / t)=a c / t u
$$

With this sum and product, the set $B$ is a ring with unity $1 / 1$, and for all $s \in S$ we have $s / 1$ and $1 / s$ are inverse to each other. Moreover, $a / s=(a / 1)(s / 1)^{-1}$. For details of this see [8, p. 118].

The mapping $a \mapsto a / 1$ of $A$ into $B$ is an injective homomorphism, and thus we can identify $A$ with its image in $B$. In this way every element $s \in S$ is invertible in $B$, and we can write $a / s=a s^{-1}$ for $a / s \in B$. Since $a \in A$ and $s \in S$, by item 4 of Definition 2.2.1 there exist $t \in S$ and $b \in A$ such that $t a=b s$, and this implies that $a s^{-1}=t^{-1} b$. Therefore, every element of $B$ can also be written as $t^{-1} b$, for $b \in A$ and $t \in S$. The ring $B$ is called the ring of quotients of $A$ defined by $S$.

Let $S$ be the set of elements of $A$ which are not zero divisors of $A$. When $S$ allows an arithmetic of fractions that is, when $S$ verifies items 4 and 5 of Definition 2.2.1 then the ring $B$ is simply called the ring of quotients of $A$.

Example 2.2.1 ([16], Example (i) p. 44). Let $A=\mathbb{F}\langle x, y\rangle$ be the free associative algebra generated by $\{x, y\}$ over a field $\mathbb{F}$. Let $S=A \backslash\{0\}$. Then $S$ does not verify item (4) of Definition 2.2.1. To see this we can choose for example $a=x$ and $s=y$. Then we can see that there is no $b \in A$ and $t \in S$ such that $x t=y b$. 
Example 2.2.1 shows that in general the ring of quotients of an arbitrary ring does not exist. The following result gives a condition for the existence of a ring of quotients. It is a particular case of Goldie's theorem, which gives, for a ring $A$, a necessary and sufficient condition that the ring of quotients of $A$ is semisimple and artinian, for more details of this see [16, Section 2.1].

Theorem 2.2.2 (|8], Theorem 3.6.12 (iv)). Suppose that $A$ is a noetherian domain, then $A$ has a ring of quotients.

Let $A$ be a ring and let $S$ be a subset of $A$ allowing arithmetic of fractions and let $B$ be the ring of quotients of $A$ defined by $S$. Suppose that $A^{\prime}$ is another ring and $\varphi: A \rightarrow A^{\prime}$ is a homomorphism such that $\varphi(s)$ is invertible for every $s \in S$. Then we can define $\bar{\varphi}: B \rightarrow A^{\prime}$ by $\bar{\varphi}\left(a s^{-1}\right)=\varphi(a) \varphi(s)^{-1}$. We have that $\bar{\varphi}$ is a homomorphism and $\bar{\varphi}(a)=a$ for all $a \in A$. Moreover, $\bar{\varphi}$ is the unique homomorphism of $B$ into $A^{\prime}$ which extends $\varphi$. In fact, if $\psi$ is another homomorphism of $B$ into $A^{\prime}$ such that $\psi(a)=\varphi(a)$ for every $a \in A$, then $\psi\left(a s^{-1}\right)=\psi(a) \psi(s)^{-1}=\varphi(a) \varphi(s)^{-1}=\bar{\varphi}\left(a s^{-1}\right)$. Thus $\bar{\varphi}=\psi$. We have shown the following proposition.

Proposition 2.2.3 ([8], Proposition 3.6.5). Let $A$ be a ring and let $S$ be a subset of $A$ allowing arithmetic of fractions and let $B$ be the ring of quotients of $A$ defined by S. Suppose that $A^{\prime}$ is another ring and that $\varphi: A \rightarrow A^{\prime}$ is a homomorphism such that $\varphi(s)$ is invertible for every $s \in S$. Then there exists a unique homomorphism $\bar{\varphi}: B \rightarrow A^{\prime}$ which extends $\varphi$, namely $\bar{\varphi}\left(a s^{-1}\right)=\varphi(a) \varphi(s)^{-1}$.

Now, let $L$ be a finite-dimensional Lie algebra and let $U(L)$ be its a universal enveloping algebra. By Theorem 2.1.9 we have that $U(L)$ is a noetherian domain, hence Theorem 2.2 .2 implies that $U(L)$ has a ring of quotients, denoted by $Q(L)$.

Given $x \in L$ we can define $\operatorname{ad}_{U(L)} x: U(L) \rightarrow U(L)$ by $\operatorname{ad}_{U(L)} x(w)=[x, w]$. This map is a derivation of $U(L)$. The mapping $x \mapsto \operatorname{ad}_{U(L)} x$ is called the adjoint representation of $L$ in $U(L)$. Analogously we define $\operatorname{ad}_{Q(L)} x: Q(L) \rightarrow Q(L)$, by $\operatorname{ad}_{Q(L)} x(w)=[x, w]$. Here it is important to note that every element of $L$ can be identified by $x / 1$ in the ring $Q(L)$ and that products in the commutator $[x, w]=$ $x w-w x$ are products in the ring $Q(L)$. The mapping $x \mapsto \operatorname{ad}_{Q(L)} x$ is called the adjoint representation of $L$ in $Q(L)$. It is immediate to verify that $\left.\operatorname{ad}_{Q(L)} x\right|_{U(L)}=\operatorname{ad}_{U(L)} x$ and $\left.\operatorname{ad}_{Q(L)} x\right|_{L}=\operatorname{ad} x$. 


\subsection{Isomorphism between universal enveloping al- gebras of Lie algebras}

In this section, which is based mainly on the paper by Riley and Usefi [27], we state some known results about the properties of Lie algebras which are preserved by an isomorphism between their universal enveloping algebras.

Let $S$ be a subalgebra of a Lie algebra $L$. Fixing a basis $\left\{x_{i}\right\}_{i \in \mathcal{I}}$ of $S$ we can extend this to a basis $\left\{x_{i}\right\}_{i \in \mathcal{I}} \cup\left\{y_{j}\right\}_{j \in \mathcal{J}}$ of $L$. By the PBW Theorem 2.1.7 we can consider $U(S)$ as a subalgebra of $U(L)$.

Lemma 2.3.1. Let $M$ be an ideal of a Lie algebra $L$ and let $S$ be a subalgebra of $L$. Then the following hold.

1. The right ideal $M U(L)$ of $U(L)$ generated by $M$ coincides with the left ideal $U(L) M$ of $U(L)$ generated by $M$. Hence, $M U(L)=U(L) M$ is a two-sided ideal.

2. Let $\pi: L \rightarrow L / M$ be the natural homomorphism of $L$ onto $L / M$. Then the homomorphism $U(L) \rightarrow U(L / M)$ induced by $\pi$ is surjective with kernel $M U(L)$. Consequently, $U(L / M) \cong U(L) / M U(L)$.

3. $\omega(M) \omega(L)=M U(L) \omega(L)=M \omega(L)$. Consequently, $\omega(M) \omega(L)$ is a two-sided ideal.

4. $L \cap \omega^{n}(S) \omega(L)=\gamma_{n+1}(S)$. In particular, $L \cap M \omega(L)=M^{\prime}$.

Proof. For the proof of parts (1) and (2) see [8, Proposition 2.2.14], for (4) see [27, Proposition 6.1(1)] or [26]. It only remains to show part (3).

In order to show part (3) note that $\omega(M) \omega(L) \subseteq(M U(L)) \omega(L)=M \omega(L)$ and $M \omega(L) \subseteq \omega(M) \omega(L)$. Thus

$$
M \omega(L) \subseteq \omega(M) \omega(L) \subseteq M U(L) \omega(L) \subseteq M \omega(L) .
$$

Hence the lemma holds. 
By Lemma 2.3.1 (1), if $M$ is an ideal of a Lie algebra $L$, then the two-sided ideal of $U(L)$ generated by $M$ coincides with $M U(L)$ and also with $U(L) M$. Hence we will usually write this two-sided ideal as $M U(L)$ or $U(L) M$ whichever is more convenient.

The automorphism group of $U(L)$ consists of those invertible linear transformations $\alpha$ of $A$ that satisfy $\alpha(a b)=\alpha(a) \alpha(b)$ for all $a, b \in A$, and it is denoted by Aut $(U(L))$. The following result reflects the importance of the ideal of $U(L)$ generated by the derived algebra $L^{\prime}$. In Chapter 2 we will quote this result several times.

Lemma 2.3.2 ([4], Prop. 2.1). The ideal $L^{\prime} U(L)$ coincides with the two-sided ideal of $U(L)$ generated by $\{[a, b]=a b-b a \mid a, b \in U(L)\}$. Consequently, $L^{\prime} U(L)$ is invariant under $\operatorname{Aut}(U(L))$.

It is known that two non-isomorphic Lie algebras may have isomorphic universal enveloping algebras, see Example 2.1.6. Nevertheless, several properties of a Lie algebra are determined by the isomorphism type of its universal enveloping algebra. For instance, it is a well known that if $L$ is a finite-dimensional Lie algebra, then the (linear) dimension of $L$ coincides with the Gelfand-Kirillov dimension of $U(L)$ (see [16, 8.1.15(iii)]). Thus $\operatorname{dim} L$ is determined only by the isomorphism type of $U(L)$. Riley and Usefi in 27] presented a detailed study of some properties of Lie algebras that are determined by the isomorphism type of its universal enveloping algebra. In the following lemma we give a summary of the ones that we use in this thesis.

Lemma 2.3.3. Let $L$ and $H$ be Lie algebras and $\alpha: U(L) \rightarrow U(H)$ an algebra isomomorphim, and suppose that $L$ is finite-dimensional. Then the following hold.

1. $\operatorname{dim} L=\operatorname{dim} H$ and $\operatorname{dim} L / L^{\prime}=\operatorname{dim} H / H^{\prime}$.

2. If $L$ is nilpotent, then so is $H$. Moreover, in this case the nilpotency classes of $L$ and $H$ coincide.

3. $L^{\prime} / L^{\prime \prime} \cong H^{\prime} / H^{\prime \prime}$; in particular, $\operatorname{dim} L^{\prime} / L^{\prime \prime}=\operatorname{dim} H^{\prime} / H^{\prime \prime}$.

4. If $L$ is metabelian, then so is $H$.

5. If $L$ is solvable, then so is $H$. 
6. If $M$ and $N$ are ideals of $L$ and $H$, respectively; such that $\alpha(M U(L))=N U(H)$. Then, $M / M^{\prime} \cong N / N^{\prime}$.

Proof. For item (1) see [16, 8.1.15(iii)] and [27, Corollary 4.2], respectively. The proof of (2) can be found in 27, Proposition 4.3]. For items (3) and (4) see [27, Corollary 7.2]; (5) is proved in [27, Proposition 7.4] and finally (6) is proved in 27, Corollary $6.2]$.

We note that item (1) of Lemma 2.3.3 implies that the isomorphism problem has a positive solution for finite-dimensional abelian Lie algebras. In fact, let $L, H$ be finite-dimensional abelian Lie algebras such that $U(L) \cong U(H)$, then $\operatorname{dim} L=\operatorname{dim} H$, and so $L \cong H$.

The next result is a useful tool. It basically says that we can assume that an isomorphism between universal enveloping algebras preserves the augmentation ideals. This result is a variation of [27, Lemma 2.1].

Lemma 2.3.4. Let $L$ and $H$ be Lie algebras and suppose that $\alpha: U(L) \rightarrow U(H)$ is an isomorphism. Then there exists an isomorphism $\bar{\alpha}: U(L) \rightarrow U(H)$ such that

1. $\bar{\alpha}(\omega(L))=\omega(H) ;$ and

2. if $x \in L$ such that $\alpha(x) \in \omega(H)$, then $\bar{\alpha}(x)=\alpha(x)$.

Proof. Let $\varepsilon_{H}$ be the augmentation map on $U(H)$. Define the map $\tilde{\alpha}: L \rightarrow U(L)$ by $\tilde{\alpha}(x)=\alpha(x)-\varepsilon_{H}(\alpha(x))$ for all $x \in L$. Then $\tilde{\alpha}$ is a Lie algebra homomorphism, and so it can be extended to be an algebra homomorphism $\bar{\alpha}: U(L) \rightarrow U(H)$. By [27, Lemma 2.1], $\bar{\alpha}: U(L) \rightarrow U(H)$ is an algebra isomorphism which satisfies property (1). The definition of $\bar{\alpha}$ guarantee that it also satisfies property (2).

\subsection{Hopf algebras}

The present section is devoted to define a very interesting structure and some of its properties, the Hopf algebras. This structure is very important in the study of $U(L)$, because $U(L)$ itself is a Hopf algebra.

In short, a Hopf algebra is a structure that is simultaneously an algebra and a coalgebra, with a notion of compatibility making it a bialgebra. Moreover, a Hopf 
algebra is equipped with an antiautomorphism satisfying a certain property. For an introduction to this theory we cite Sweedler's book [31] for a pleasant reading, and for a more specialized treatment we refer to Montgomery's book [19] or the paper by Milnor and Moore [17.

Let $A$ be an associative algebra over $\mathbb{F}$ with identity 1 . One can view the multiplication on $A$ as a linear map $m: A \otimes A \rightarrow A$. Further, using the identity, one can define the linar map $u: \mathbb{F} \rightarrow A$ by $u(\alpha)=\alpha \cdot 1$ for all $\alpha \in \mathbb{F}$. In this language the fact that $A$ is an associative algebra is equivalent to the fact that the following two diagrams commute:
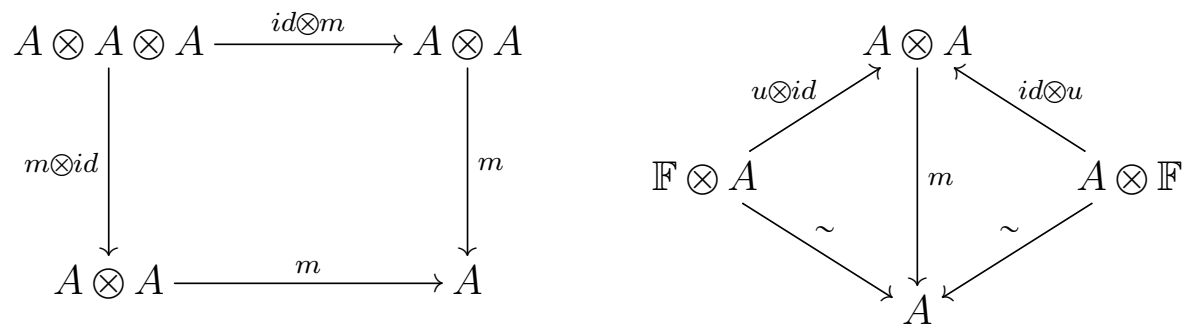

In these diagrams, and in the following diagrams, $i d$ denotes an identity map. The symbol $\sim$ denotes the isomorphism $\sim: \mathbb{F} \otimes A \rightarrow A$ or $A \otimes \mathbb{F} \rightarrow A$ defines by $\alpha \otimes a \mapsto \alpha a$ or $a \otimes \alpha \rightarrow \alpha a$, respectively. In this way we can view an associative algebra as a tuple $(A, m, u)$ such that the diagrams above commute.

An advantage of this approach is that it is categorical. Hence we can construct the dual of this concept, and thus we obtain the definition of a coalgebra.

Definition 2.4.1. A coalgebra is a tuple $(C, \Delta, \varepsilon)$, where $C$ is a vector space, $\Delta$ : $C \rightarrow C \otimes C$ and $\varepsilon: C \rightarrow \mathbb{F}$ are linear maps, such that the following diagrams commute:
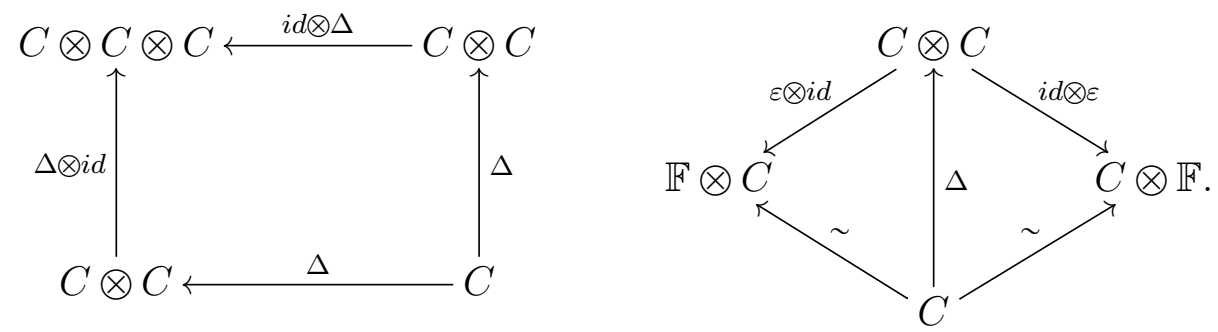

Let $A, B$ be two associative algebras over $\mathbb{F}$ and let $f: A \rightarrow B$ be an algebra homomorphism, that is $f(x y)=f(x) f(y)$ for all $x, y \in A$. If we consider these two 
associative algebras as the tuples $\left(A, m_{A}, u_{A}\right)$ and $\left(B, m_{B}, u_{B}\right)$, then a linear map $f: A \rightarrow B$ is an algebra homomorphism if and only if the following two diagrams commute:
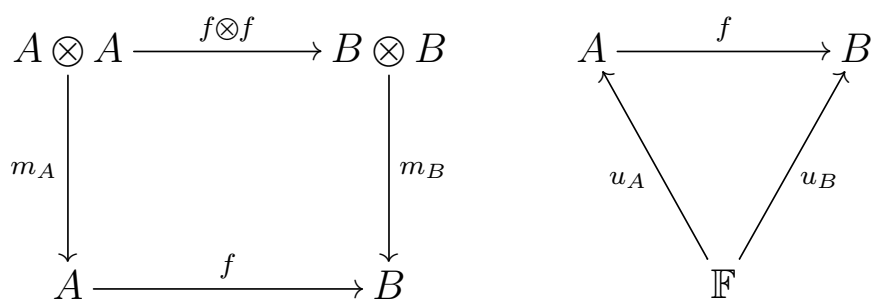

The dual concept of an algebra homomorphism is the concept of a coalgebra homomorphism and it is defined as follows.

Definition 2.4.2. Let $\left(C, \Delta_{C}, \varepsilon_{C}\right)$ and $\left(D, \Delta_{D}, \varepsilon_{D}\right)$ be two coalgebras. A linear map $g: C \rightarrow D$ is a coalgebra homomorphism if the following diagrams commute:
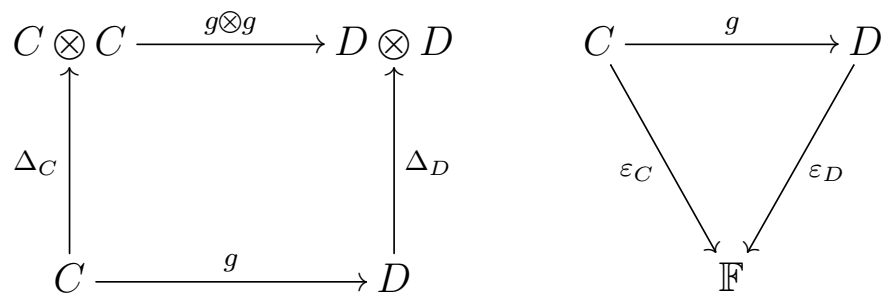

If $A$ is an algebra, then $A \otimes A$ is also an algebra with multiplication defined by $(a \otimes b)(c \otimes d)=(a c) \otimes(b d)$. Similarly, if $A$ is a coalgebra, then $A \otimes A$ is a coalgebra with comultiplication defined analogously.

Definition 2.4.3. A bialgebra is a tuple $(H, m, u, \Delta, \varepsilon)$, such that $(H, \Delta, \varepsilon)$ is a coalgebra, $(H, m, u)$ is an algebra and the maps $\Delta: H \rightarrow H \otimes H$ and $\varepsilon: C \rightarrow \mathbb{F}$ are algebra homomorphisms.

The condition that $\Delta: H \rightarrow H \otimes H$ and $\varepsilon: C \rightarrow \mathbb{F}$ are algebra homomorphisms is equivalent to the condition that the maps $m: H \otimes H \rightarrow H$ and $u: \mathbb{F} \rightarrow H$ are coalgebra homomorphisms, and this condition is a connection between the structure of an algebra and a coalgebra.

Definition 2.4.4. Let $\left(H_{1}, m_{1}, u_{1}, \Delta_{1}, \varepsilon_{1}\right)$ and $\left(H_{2}, m_{2}, u_{2}, \Delta_{2}, \varepsilon_{2}\right)$ be two bialgebras. A linear map $f: H_{1} \rightarrow H_{2}$ is a bialgebra homomorphism if it is simultaneously an algebra and coalgebra homomorphism. 
Definition 2.4.5. A Hopf algebra is a tuple $(H, m, u, \Delta, \varepsilon, S)$, where $(H, m, u, \Delta, \varepsilon)$ is a bialgebra and $S: H \rightarrow H$ is a linear mapping verifying the identity

$$
u \varepsilon(h)=\sum S\left(h_{i_{1}}\right) h_{i_{2}}=\sum h_{i_{1}} S\left(h_{i_{2}}\right),
$$

for all $h \in H$, where $\Delta(h)=\sum h_{i_{1}} \otimes h_{i_{2}}$.

\section{Example 2.4.1.}

1. Let $G$ be group and let $\mathbb{F} G$ be its group algebra. Define $\Delta(g)=g \otimes g$ and $\varepsilon(g)=1$ for $g \in G$. Extending them linearly, we obtain that $(\mathbb{F} G, \Delta, \varepsilon)$ is a coalgebra. We note that $\Delta$ and $\varepsilon$ are algebra homomorphisms. Thus $\mathbb{F} G$ is a bialgebra. Defining $S: \mathbb{F} G \rightarrow \mathbb{F} G$ by $g \mapsto g^{-1}$, we have that $\mathbb{F} G$ is a Hopf algebra.

2. Let $U(L)$ be the universal enveloping algebra of a Lie algebra $L$. Define $\Delta(x)=$ $1 \otimes x+x \otimes 1$ and $\varepsilon(x)=0$ for $x \in L$. Using the universal property of $U(L)$, we have that $U(L)$ is a bialgebra. Defining $S(x)=-x$ for $x \in L$, and using again the universal property of $U(L)$, we have that $U(L)$ is a Hopf algebra.

A subspace $V$ of a Hopf algebra $H$ is called Hopf ideal of $H$ if it is a kernel of a bialgebra homomorphism and $S(V) \subseteq V$. In [27, Lemma 8.3] it is shown that $M U(L)$ is a Hopf ideal of $U(L)$ and also that $U(L) / M U(L) \cong U(L / M)$ as Hopf algebras (cf Lemma 2.3.1 (2)).

Let $C$ be a coalgebra. An element $c \in C$ is called group-like if $\Delta c=c \otimes c$ and $\varepsilon(c)=1$. The set of group-like elements is denoted by $G(C)$. The set $G(C)$ is linearly independent in $C$ [31, Proposition 3.2.1]. Consequently, if $K$ is a group then $G(\mathbb{F} K)=K$. Let $H$ be a bialgebra, an element $x \in H$ is called primitive if $\Delta x=x \otimes 1+1 \otimes x$. The set of primitive elements of $H$ is denoted by $\mathcal{P}(H)$, and it is a Lie algebra with the bracket $[x, y]=x y-y x$.

One of the most important results about the Hopf algebra structure of $U(L)$ says that in characteristic zero the structure of $L$ is determined by the bialgebra structure of $U(L)$. The proof of this fact relies on the following proposition whose proof can be found in the book of Montgomery [19]. Before stating this result we need to introduce a notation. Given an integer $p$ and a subset $X$ of an associative algebra $A$, we denote by $X_{p}$ the subspace generated by the set $\left\{x^{p^{k}} \mid x \in X, k \geqslant 0\right\}$. 
Proposition 2.4.2 ([19], Proposition 5.5.3). Let $L$ be a Lie algebra. Then the following statements hold:

1. $G(U(L))=\{1\}$;

2. if $\operatorname{char} \mathbb{F}=0$, then $\mathcal{P}(U(L))=L$;

3. if $\operatorname{char} \mathbb{F}=p$, then $\mathcal{P}(U(L))=L_{p}$.

Let $H_{1}, H_{2}$ be two bialgebras, $f: H_{1} \rightarrow H_{2}$ a bialgebra homomorphism and $x \in \mathcal{P}\left(H_{1}\right)$. Then

$$
\begin{aligned}
\Delta_{H_{2}}(f(x)) & =(f \otimes f) \Delta_{H_{1}}(x) \\
& =(f \otimes f)(1 \otimes x+x \otimes 1) \\
& =1 \otimes f(x)+f(x) \otimes 1 .
\end{aligned}
$$

Thus $f\left(\mathcal{P}\left(H_{1}\right)\right) \subseteq \mathcal{P}\left(H_{2}\right)$. If $f$ is a bialgebra isomorphism then a symmetric argument implies that $f^{-1}\left(\mathcal{P}\left(H_{2}\right)\right) \subseteq \mathcal{P}\left(H_{1}\right)$, and consequently $f\left(\mathcal{P}\left(H_{1}\right)\right)=\mathcal{P}\left(H_{2}\right)$. With this in mind we can state an immediate corollary of Proposition 2.4.2.

Corollary 2.4.3. Let $L, H$ be two Lie algebras such that $U(L) \cong U(H)$ as bialgebras. Then

1. if $\operatorname{char} \mathbb{F}=0$, then $L \cong H$;

2. if $\operatorname{char} \mathbb{F}=p$, then $L_{p} \cong H_{p}$.

Example 2.4.4. Let $\mathbb{F}$ be a field with $\operatorname{char} \mathbb{F}=2$. We consider two Lie algebras of dimension 5 over $\mathbb{F}$

$$
\begin{aligned}
& L=\left\langle x_{1}, x_{2}, x_{3}, x_{4}, x_{5} \mid\left[x_{1}, x_{2}\right]=x_{4},\left[x_{1}, x_{4}\right]=x_{5}\right\rangle, \\
& H=\left\langle x_{1}, x_{2}, x_{3}, x_{4}, x_{5} \mid\left[x_{1}, x_{2}\right]=x_{4},\left[x_{1}, x_{4}\right]=x_{5},\left[x_{2}, x_{3}\right]=x_{5}\right\rangle .
\end{aligned}
$$

In Example 2.1.6, we saw that the homomorphism $f: U(L) \rightarrow U(H)$, induced by $x_{1} \mapsto x_{1}, x_{2} \mapsto x_{2}, x_{3} \mapsto x_{3}+x_{1}^{2}, x_{4} \mapsto x_{4}$ and $x_{5} \mapsto x_{5}$ is an algebra isomorphism between $U(L)$ and $U(H)$. In order to show that $f$ is a coalgebra homomorphism it is enough verify that

$$
(f \otimes f) \Delta_{L}\left(x_{i}\right)=\Delta_{H}\left(f\left(x_{i}\right)\right)
$$


for every $x_{i}$ in the basis of $L$. By definition of $f$, we have that $(f \otimes f) \Delta_{L}\left(x_{i}\right)=$ $\Delta_{H}\left(f\left(x_{i}\right)\right)$, for $i \neq 3$. For $x_{3}$ we have

$$
\begin{aligned}
(f \otimes f) \Delta_{L}\left(x_{3}\right) & =1 \otimes f\left(x_{3}\right)+f\left(x_{3}\right) \otimes 1 \\
& =1 \otimes\left(x_{3}+x_{1}^{2}\right)+\left(x_{3}+x_{1}^{2}\right) \otimes 1 \\
& =1 \otimes x_{3}+x_{3} \otimes 1+\left(1 \otimes x_{1}^{2}+x_{1}^{2} \otimes 1\right) \\
& =\Delta_{H}\left(x_{3}\right)+\Delta_{H}\left(x_{1}^{2}\right) \\
& =\Delta_{H}\left(x_{3}+x_{1}^{2}\right) .
\end{aligned}
$$

Note that in the fourth equality we used that $\mathcal{P}(U(H))=H_{2}$, see Proposition 2.4.2(3). Thus $f$ is a bialgebra isomorphism. But, it is not true that $L \cong H$, since $Z(L)=$ $\left\langle x_{3}, x_{5}\right\rangle$ and $Z(H)=\left\langle x_{5}\right\rangle$. However, by Corollary 2.4 .3 we have that $L_{2} \cong H_{2}$.

This example shows that if $\mathbb{F}$ is a field of characteristic $p$ and $U(L) \cong U(H)$ as bialgebras, then we do not necessarily have $L \cong H$. That is, the bialgebra structure of $U(L)$ does not necessarily determine the structure of $L$. 


\section{Chapter 3}

\section{Solvable Lie algebras of dimension at most 4}

\subsection{The classification by de Graaf}

In this section, we reproduce the classification of solvable Lie algebras of dimension at most 4, over an arbitrary field, given by de Graaf [5]. In dimension 3 this classification is the same as that given by Patera and Zassenhaus [23], but de Graaf gives a shorter description of these algebras. Recall that only the non-zero brackets are displayed in multiplication tables of Lie algebras.

Theorem 3.1.1 ([5]). Let $L$ be a 3-dimensional solvable Lie algebra over a field $\mathbb{F}$. Then $L$ is isomorphic to one of the following algebras:

1. $L^{1}=\left\langle x_{1}, x_{2}, x_{3}\right\rangle$ (the abelian Lie algebra);

2. $L^{2}=\left\langle x_{1}, x_{2}, x_{3} \mid\left[x_{3}, x_{1}\right]=x_{1},\left[x_{3}, x_{2}\right]=x_{2}\right\rangle$;

3. $L_{a}^{3}=\left\langle x_{1}, x_{2}, x_{3} \mid\left[x_{3}, x_{1}\right]=x_{2},\left[x_{3}, x_{2}\right]=a x_{1}+x_{2}\right\rangle$ with some $a \in \mathbb{F}$;

4. $L_{a}^{4}=\left\langle x_{1}, x_{2}, x_{3} \mid\left[x_{3}, x_{1}\right]=x_{2},\left[x_{3}, x_{2}\right]=a x_{1}\right\rangle$ with some $a \in \mathbb{F}$.

If $L_{j}^{i} \cong L_{v}^{u}$ then $i=u$. Moreover $L_{a}^{3} \cong L_{b}^{3}$ if and only if $a=b$ and $L_{a}^{4} \cong L_{b}^{4}$ if and only if there is an $\alpha \in \mathbb{F}^{\times}$with $a=\alpha^{2} b$.

In dimension four we have 14 families of isomorphism classes of solvable Lie algebras. We group these Lie algebras into five groups in such a way that two Lie 
algebras in distinct groups cannot have isomorphic universal enveloping algebras, see Proposition 3.2.2. We follow the notation introduced by de Graaf [5]. Several of de Graaf's families, such as $M_{a}^{3}, M_{a}^{13}, M_{a, b}^{6}$ and $M_{a, b}^{7}$, are split between two groups.

The 4-dimensional solvable Lie algebras are presented in tables, each table corresponding to one group. Each table contains six columns and the information contained in the columns can be described as follows.

name: The name of the algebra in the notation of de Graaf [5].

relations: The non-trivial products in the multiplication table of the algebra with respect to a basis $\left\{x_{1}, x_{2}, x_{3}, x_{4}\right\}$.

char: Restrictions on the characteristic of the field over which the algebra is defined. If this entry is blank, then the algebra is defined over an arbitrary field.

parameters: The necessary parameters to define the Lie algebra

isomorphism: The conditions under which isomorphism occurs between two algebras in the same family. If the family is described by a single parameter $a$, then the condition given in this entry is equivalent to the isomorphism $M_{a}^{i} \cong M_{b}^{i}$. If the family is described by two parameters $a$ and $b$, then the condition given in this entry is equivalent to the isomorphism $M_{a, b}^{i} \cong M_{c, d}^{i}$.

center: The center of the corresponding Lie algebra.

By the classification of de Graaf [5], there is no isomorphism between two Lie algebras in different families, while two Lie algebras belonging to the same family are isomorphic if and only if the condition in the Isomorphism column of the corresponding line of the table holds.

Group 1: The abelian Lie algebra. The first group contains only the abelian Lie algebra.

\begin{tabular}{|c|c|c|c|c|c|}
\hline Name & relations & char & parameter & isomorphism & center \\
\hline$M^{1}$ & & & & & $M^{1}$ \\
\hline
\end{tabular}



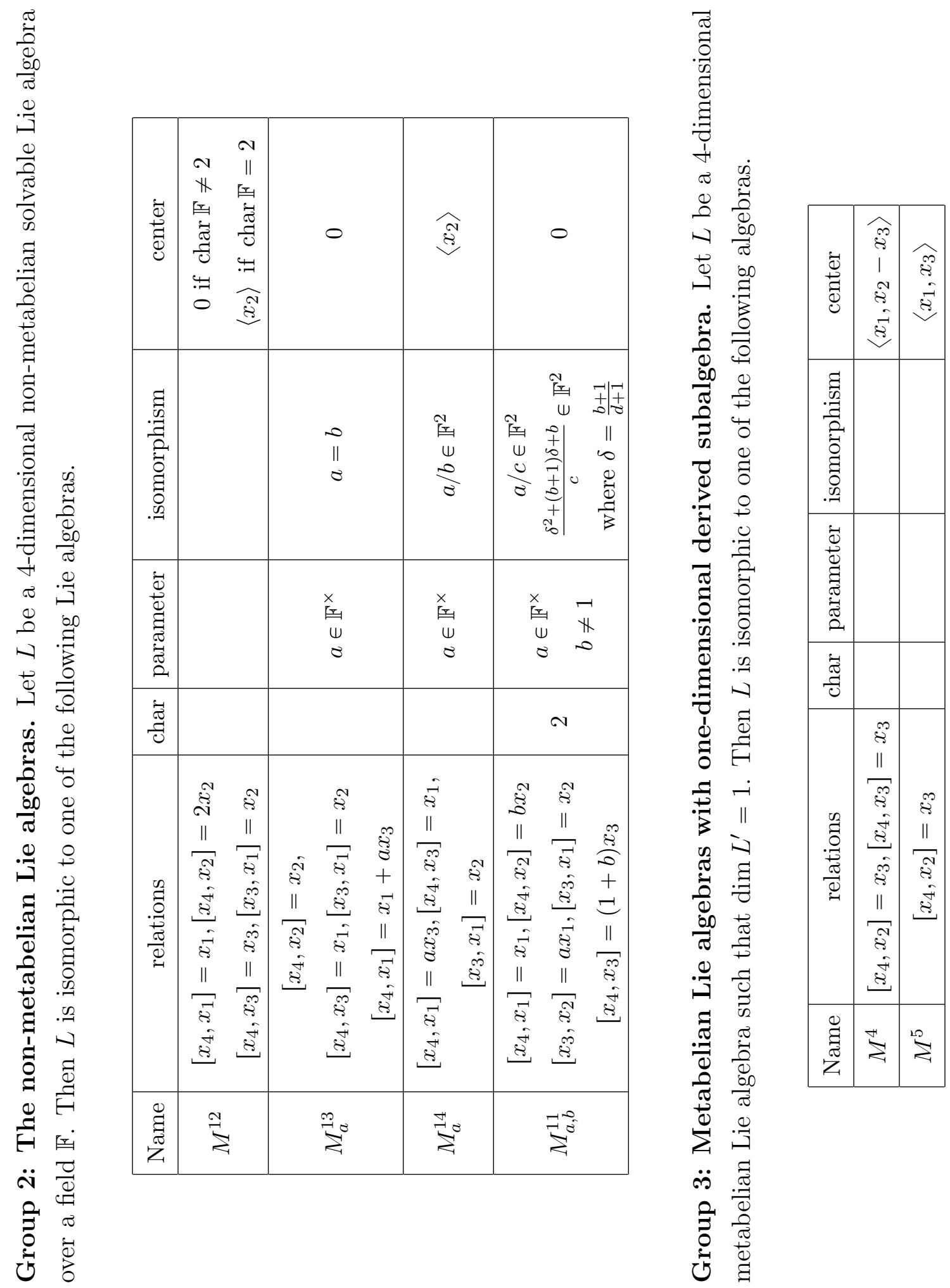
Group 4: Metabelian Lie algebras with three-dimensional derived subalgebra. Suppose that $L$ is a 4-dimensional metabelian Lie algebra with $\operatorname{dim} L^{\prime}=3$. Then $L$ is isomorphic to one of the following Lie algebras.

\begin{tabular}{|c|c|c|c|c|c|}
\hline Name & relations & char & parameter & isomorphism & center \\
\hline$M^{2}$ & $\begin{array}{c}{\left[x_{4}, x_{1}\right]=x_{1},\left[x_{4}, x_{2}\right]=x_{2},} \\
{\left[x_{4}, x_{3}\right]=x_{3}}\end{array}$ & & & 0 \\
\hline \multirow{2}{*}{$M_{a}^{3}$} & {$\left[x_{4}, x_{1}\right]=x_{1},\left[x_{4}, x_{2}\right]=x_{3}$,} & & $a \in \mathbb{F}^{*}$ & $a=b$ & 0 \\
& {$\left[x_{4}, x_{3}\right]=-a x_{2}+(a+1) x_{3}$} & & & & \\
\hline$M_{a, b}^{6}$ & {$\left[x_{4}, x_{1}\right]=x_{2},\left[x_{4}, x_{2}\right]=x_{3}$,} & & $a \in \mathbb{F}^{\times}$ & $a=c$ & 0 \\
& {$\left[x_{4}, x_{3}\right]=a x_{1}+b x_{2}+x_{3}$} & & $b \in \mathbb{F}$ & $b=d$ & 0 \\
& {$\left[x_{4}, x_{1}\right]=x_{2},\left[x_{4}, x_{2}\right]=x_{3}$,} & & $a \in \mathbb{F}^{\times}$ & $a=\alpha^{3} c$ & \\
$M_{a, b}^{7}$ & {$\left[x_{4}, x_{3}\right]=a x_{1}+b x_{2}$} & & $b \in \mathbb{F}$ & $b=\alpha^{2} d$ & 0 \\
& & & & with $\alpha \in \mathbb{F}^{\times}$ & \\
\hline
\end{tabular}




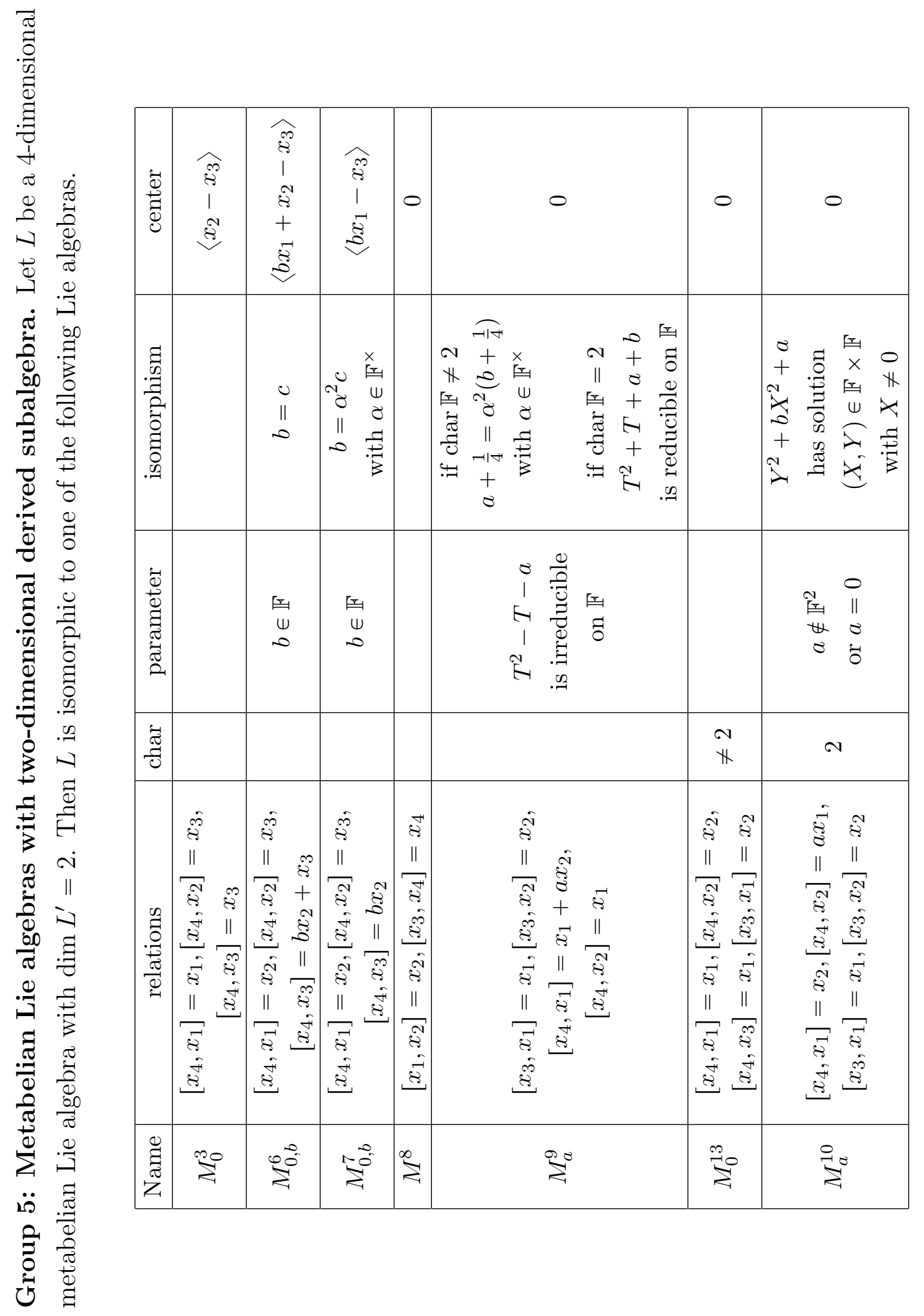


This description has only one difference with that given by de Graaf. In [5] the family $M_{a}^{10}$ is considered with $a \notin \mathbb{F}^{2}$. But, since $M_{a}^{10} \cong M_{0}^{13}$ if char $\mathbb{F}=2$ (see [5. Section 5]) we added to the family $M_{a}^{10}$ the parameter $a=0$, and omitted this from the family $M_{a}^{13}$ when $\operatorname{char} \mathbb{F}=2$.

\subsection{The first step}

This section is devoted to a first analysis of the isomorphism problem for solvable Lie algebras of dimension at most 4 . In dimension 3 we have that the isomorphism problem has a positive solution. Originally this was shown by Chun, Kajiwara and Lee in [4]; they showed that the isomorphism problem has a positive solution in the class of 3-dimensional Lie algebra over a field of characteristic different from two. But we observe that in the solvable case this affirmation is independent of the characteristic of the field. We will see an alternative proof of this fact using the theory developed in Chapters 4 and 5.

The main result of this thesis is the following theorem.

Theorem 3.2.1. Let $L, H$ be solvable Lie algebras of dimension 4 over a field of characteristic 0 . If $U(L) \cong U(H)$, then $L \cong H$.

Theorem 3.2.1 basically states that the isomorphism problem has a positive solution for solvable Lie algebras of dimension 4 over a field of characteristics 0 . In this chapter, we cannot give a complete proof of Theorem 3.2.1. However, we can explain the main idea of our proof and present a part of it. The proof of Theorem 3.2.1 consists basically of two steps. First, using the classification by de Graaf we divide the Lie algebras into five groups (this division is already done in Section 3.1) in a way that two Lie algebras in different groups cannot have isomorphic universal enveloping algebras. Second, we analyze each group in order to ensure that in the same group do not exist two non-isomorphic Lie algebras with isomorphic universal enveloping algebras.

The following lemma justifies the division made in Section 3.1 and completes the first step of the proof of Theorem 3.2.1. 
Proposition 3.2.2. Suppose that $L$ and $H$ are 4-dimensional solvable Lie algebras over an arbitrary field such that $L$ and $H$ belong to distinct groups in the group division in Section 3.1. Then $U(L) \nRightarrow U(H)$.

Proof. Lemma 2.3.3 implies that the isomorphism type of $U(L)$ determines whether $L$ is abelian, whether $L$ is metabelian, and $\operatorname{dim} L^{\prime}$. Therefore, by the form of the group division in Section 3.1, it is impossible that $U(L) \cong U(H)$ for $L$ and $H$ in different groups.

The following lemma is a partial result towards the second step of the proof of Theorem 3.2.1.

Lemma 3.2.3. $U\left(M^{4}\right) \nRightarrow U\left(M^{5}\right)$ holds over an arbitrary field $\mathbb{F}$.

Proof. Note that $\gamma_{n}\left(M^{4}\right)=\left\langle x_{3}\right\rangle$ for $n \geqslant 3$ and $\gamma_{3}\left(M^{5}\right)=0$. The proof follows at once from Lemma 2.3.3 (2), as $M^{4}$ is non-nilpotent, but $M^{5}$ is nilpotent.

The following chapters focus mainly (but not exclusively) on the development of the techniques and methods that will help us complete the second step of the proof of Theorem 3.2.1. 


\section{Chapter 4}

\section{Center, semicenter and Frobenius semiradical}

In this chapter, we will make a brief study of three objects which are very important to better understand the structure of the universal enveloping algebra. These objects are the center, the semicenter and the Frobenius semiradical. The first two are abelian subalgebras of the universal enveloping algebra, and they are preserved by any isomorphism between universal enveloping algebras. The third is a characteristic subalgebra of a Lie algebra and it is very related to the first two objects.

Section 4.1 is dedicated to the study of the center of the universal enveloping algebra. There exist many results about this object. The study presented here is based on some observations that help us in the study of the isomorphism problem. Section 4.2 is devoted to a construction of counterexamples for the isomorphism problem in prime characteristic. This section is inspired by the analysis of several examples of the paper by Schneider and Usefi [29]. In Section 4.3, we study the semicenter of a universal enveloping algebra. We define this object and we will show that it is preserved by any isomorphism between universal enveloping algebras. The references for these two sections are Dixmier's book [8], the paper by Braun and Vernik [3] and the paper by Delvaux, Nauwelaerts and Ooms [7]. Finally, in Section 4.4, we define the Frobenius semiradical of a Lie algebra and we study the relation of this with the center of the universal enveloping algebra. We believe that this section has two main results. The first is Theorem 4.4.8, which says basically that in some class of Lie algebras the dimension of the center of Lie algebra is determined by its universal enveloping algebra. 
The second is Proposition 4.4.12, which is a partial result towards the second step of the proof of Theorem 3.2.1. The principal reference for Section 4.4 is the paper by Ooms 21.

Throughout this chapter we assume that $\mathbb{F}$ is a field which has an arbitrary characteristic unless otherwise stated, $L$ is a finite-dimensional Lie algebra over $\mathbb{F}$ unless otherwise stated. The dual space of a vector space $V$ will be denote by $V^{*}$.

\subsection{Center of $U(L)$}

The center of the universal enveloping algebra $U(L)$ of a Lie algebra $L$ has a very important role in the study of the structure of $U(L)$. In fact, the study of the center is independent of the isomorphism problem, but they have an important relationship. For this reason we dedicate one section to the study of the center.

Let $L$ be a Lie algebra over a field $\mathbb{F}$ and let $U(L)$ be its universal enveloping algebra. The center of $U(L)$ is the set

$$
Z(U(L)):=\{u \in U(L) \mid u v=v u \text { for all } v \in U(L)\}
$$

Equivalently $Z(U(L))=\{u \in U(L) \mid[u, v]=0$ for all $v \in U(L)\}$. Also we can view $Z(U(L))$ as the centralizer of $L$ in $U(L)$, that is

$$
Z(U(L))=C_{U(L)}(L)=\{u \in U(L) \mid[u, x]=0 \text { for all } x \in L\}
$$

It is immediate that $Z(L) \subseteq Z(U(L))$. The subalgebra of $U(L)$ generated by $Z(L)$ can be viewed as $U(Z(L))$, and it is contained in $Z(U(L))$. The converse inclusion $Z(U(L)) \subseteq U(Z(L))$ is not true in general, as we will see in Example 4.1.1. However, if char $\mathbb{F}=0$ there exists a class of Lie algebras which verify the equality $U(Z(L))=Z(U(L))$, and we will study these Lie algebras in Section 4.4 .

Example 4.1.1. Let $H=\left\langle x_{1}, x_{2}, x_{3} \mid\left[x_{1}, x_{2}\right]=x_{3},\right\rangle$ be the 3-dimensional Heisenberg Lie algebra over a field $\mathbb{F}$, see Example 2.1.5 (4). We have that $Z(H)=\left\langle x_{3}\right\rangle$. If char $\mathbb{F}=0$ then $Z(U(H))=\mathbb{F}\left[x_{3}\right]$ (see Example 4.4.7), and this implies that $U(Z(H))=Z(U(H))$. On other hand, if char $\mathbb{F}=p$ then using equation (2.1) and the fact that $H$ is nilpotent of class two, we have that $x_{1}^{p}, x_{2}^{p} \in Z(U(H))$. Thus $\mathbb{F}\left[x_{1}^{p}, x_{2}^{p}, x_{3}\right] \subseteq Z(U(H))$, and it follows that $Z(U(H)) \nsubseteq U(Z(H))$. 
Example 4.1.1 also shows that the structure of $Z(U(L))$ depends on the characteristic of the field $\mathbb{F}$. This complicates the study of the center. Many of the existing results about of the structure of the center $Z(U(L))$ are in characteristic 0 , and there are fewer results in prime characteristic.

Let $\left\{x_{1}, x_{2}, \ldots, x_{n}\right\}$ be a basis of the Lie algebra $L$ over an arbitrary field $\mathbb{F}$. Set $U_{0}(L)=\mathbb{F}$ and let $U_{k}(L)$ for $k \geqslant 1$ denote the vector space generated by the products $x_{i_{1}} x_{i_{2}} \cdots x_{i_{r}}$, where $r \leqslant k$. It follows immediately that $U_{k}(L) U_{l}(L) \subseteq U_{k+l}(L)$ and $\bigcup_{k \geqslant 0} U_{k}(L)=U(L)$. The following lemma is a consequence of the PBW Theorem and we will use this to study $Z(U(L))$.

Lemma 4.1.2 ([13], Chapter V, Lemma 4). Let $\left\{x_{1}, x_{2}, \ldots, x_{n}\right\}$ be a basis of L. Suppose that for each $x_{i}$ there exist a positive integer $m_{i}$ and an element $z_{i} \in Z(U(L))$ such that $v_{i}=x_{i}^{m_{i}}-z_{i} \in U_{m_{i}-1}(L)$. Then, the elements of the form

$$
z_{1}^{b_{1}} z_{2}^{b_{2}} \cdots z_{n}^{b_{n}} x_{1}^{a_{1}} x_{2}^{a_{2}} \cdots x_{n}^{a_{n}}
$$

such that $b_{j} \geqslant 0,0 \leqslant a_{j}<m_{i_{j}}$, form a basis of $U(L)$.

Suppose that char $\mathbb{F}=p$ and that $L$ is a finite-dimensional Lie algebra over $\mathbb{F}$ with basis $\left\{x_{1}, x_{2}, \ldots, x_{n}\right\}$. Then, for each $i$ there exists a polynomial $f_{i}(X)=X^{p^{m_{i}}}+$ $a_{1} X^{p^{m_{i}-1}}+\cdots+a_{m} X$, such that $z_{i}=f_{i}\left(x_{i}\right) \in Z(U(L))$, see 13 , Chapter VI, Lemma 5]. Thus $z_{i}=x_{i}^{p^{m_{i}}}+v_{i}$ is in $Z(U(L))$, where $v_{i} \in U_{p^{m_{i-1}}}(L)$. Hence, by Lemma 4.1.2. the elements

$$
z_{1}^{b_{1}} z_{2}^{b_{2}} \cdots z_{n}^{b_{n}} x_{1}^{a_{1}} x_{2}^{a_{2}} \cdots x_{n}^{a_{n}}
$$

$b_{i} \geqslant 0,0 \leqslant a_{i}<p^{m^{i}}$ form a basis for $U(L)$. Let $I$ be the left (or equivalently twosided) ideal generated by $\left\{z_{1}, z_{2}, \ldots, z_{n}\right\}$. We will show that $I$ is a residually nilpotent ideal, using the following theorem proved by Hochschild.

Theorem 4.1.3 ([10], Theorem 2). Let L be a finite-dimensional Lie algebra over a field $\mathbb{F}$ of characteristic $p$ and let $C=Z(U(L)) \cap \omega(L)$. Then $\bigcap_{k \geqslant 1} U(L) C^{k}=0$; that is, the ideal $U(L) C$ is residually nilpotent.

Lemma 4.1.4. Let $L$ be a finite-dimensional Lie algebra over a field $\mathbb{F}$ of characteristic $p$ and let $I$ be the ideal defined before Theorem 4.1.3. Then I is residually nilpotent and the quotients $U(L) / I^{k}$ and $U(L) / U(L) C^{k}$ are finite-dimensional for all $k \geqslant 1$. 
Proof. Suppose that $\left\{x_{1}, \ldots, x_{n}\right\}$ is a basis of $L$ and let $\left\{z_{1}, \ldots, z_{n}\right\}$ be as above. Let $C=Z(U(L)) \cap \omega(L)$. Since $z_{i} \in C$ for all $i=1, \ldots, n$ it follows that $I \subseteq U(L) C$, and consequently $I^{k} \subseteq U(L) C^{k}$ for all $k \geqslant 1$. Theorem 4.1 .3 implies that $\bigcap_{k \geqslant 1} I^{k}=0$, and it follows that $I$ is residually nilpotent.

Now, since $I$ is the ideal generated by the set $\left\{z_{1}, z_{2}, \ldots z_{n}\right\}$ it is clear that the subset consisting of the elements 4.2 with $b_{1}+\cdots+b_{n} \geqslant 1$ is basis of $I$. Hence the cosets of the elements $x_{1}^{a_{1}} x_{2}^{a_{2}} \cdots x_{n}^{a_{n}}, 0<a_{i}<p^{m_{i}}$ form a basis for $U(L) / I$. Thus, $U(L) / I$ is finite-dimensional. Analogously, we can see that the subset consisting of the elements (4.2) with $b_{1}+b_{2}+\cdots+b_{n} \geqslant k$ is a basis for $I^{k}$. Therefore the cosets containing the elements $z_{1}^{b_{1}} z_{2}^{b_{2}} \cdots z_{n}^{b_{r}} x_{1}^{a_{1}} x_{2}^{a_{2}} \cdots x_{n}^{a_{n}}$ with $b_{1}+b_{2}+\cdots+b_{n}<k$ and $0<a_{i}<p^{m_{i}}$ form a basis for $U(L) / I^{k}$, and it follows that $U(L) / I^{k}$ is finitedimensional for all $k \geqslant 1$. Finally, since $I^{k} \subseteq U(L) C^{k}$ for all $k \geqslant 1$, it follows that $U(L) / U(L) C^{k}$ is finite-dimensional for all $k \geqslant 1$.

\subsection{A construction of counterexamples for the iso- morphism problem}

In this section, we study some necessary conditions for two Lie algebras have isomorphic universal enveloping algebras. As was said in the introduction to this chapter, this section was inspired by the analysis of the paper by Schneider and Usefi [29]. Schneider and Usefi describe in [29] several pairs of non-isomorphic Lie algebras with isomorphic universal enveloping algebras. We find that in many cases, such isomorphisms have a common underlying reason that is highlighted by the following theorem. It is also interesting to note, although not done in [29], that the isomorphisms given by Schneider and Usefi in [29] are coalgebra isomorphisms. Recall that we defined direct and semidirect product of Lie algebras before Lemma 2.1.3, and that the graded Lie algebra $\operatorname{gr}(L)=\bigoplus_{i \geqslant 1} \gamma_{i}(L) / \gamma_{i+1}(L)$ was defined before Example 2.1.2.

Theorem 4.2.1. Let $L=M \oplus\langle x\rangle$ and $H=N \rtimes\langle y\rangle$ be two finite-dimensional nilpotent Lie algebras, where $M$ and $N$ are ideals of $L$ and $H$, respectively. Suppose that $M \cong N$ and $\operatorname{gr}(L) \cong \operatorname{gr}(H)$, and there exists an element $u \in U(N)$ such that $\left(\operatorname{ad}_{U(H)} u\right)(N) \subseteq N$ and $\left.\operatorname{ad}_{U(H)} u\right|_{N}=\left.\operatorname{ad}_{U(H)} y\right|_{N}$. Then, there exists an isomorphsim between $U(L)$ and $U(H)$. 
Proof. Let $w=y-u$. Since $\operatorname{ad}_{U(H)} u(N) \subseteq N$ and $\left.\operatorname{ad}_{U(H)} u\right|_{N}=\left.\operatorname{ad}_{U(H)} y\right|_{N}$ we have that $[w, N]=0$. Suppose that $f: M \rightarrow N$ is an isomorphism between $M$ and $N$. Then the map $\alpha: L \rightarrow U(H)$, defined by $\alpha(z)=f(z)$ for $z \in M$ and $\alpha(x)=w$, is a Lie homomorphism. Note that $M \oplus\langle x\rangle \cong N \oplus\langle w\rangle$ via $\alpha$. By the universal property of $U(L)$ we obtain an algebra homomorphism $\bar{\alpha}: U(L) \rightarrow U(H)$ such that the diagram

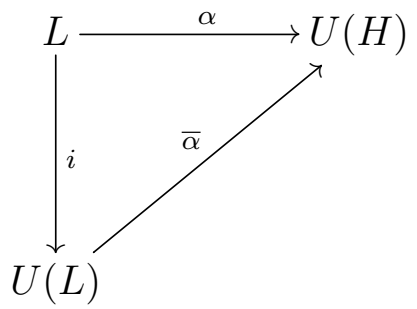

is commutative. We will show that $\bar{\alpha}$ is an isomorphism. In order to show that $\bar{\alpha}$ is surjective we note that $y=w+u \in \operatorname{Im} \bar{\alpha}$ and $N=\bar{\alpha}(M) \subseteq \operatorname{Im} \bar{\alpha}$. Therefore, the generators of $H$ are contained in $\operatorname{Im} \bar{\alpha}$, and it follows that $\bar{\alpha}(\omega(L))=\omega(H)$. Now, since $L$ is nilpotent, so is $\operatorname{gr}(L)$ and the nilpotency classes of $L$ and $\operatorname{gr}(L)$ coincide. Since $\operatorname{gr}(L) \cong \operatorname{gr}(H)$, the nilpotency classes of $L$ and $H$ also coincide and

$$
\gamma_{i}(L) / \gamma_{i+1}(L) \cong \gamma_{i}(\operatorname{gr}(L)) / \gamma_{i+1}(\operatorname{gr}(L)) \cong \gamma_{i}(\operatorname{gr}(H)) / \gamma_{i+1}(\operatorname{gr}(H)) \cong \gamma_{i}(H) / \gamma_{i+1}(H)
$$

for every $i \geqslant 1$. These isomorphisms imply that

$$
\operatorname{dim} \gamma_{i}(L) / \gamma_{i+1}(L)=\operatorname{dim} \gamma_{i}(H) / \gamma_{i+1}(H)
$$

for every $i \geqslant 1$. By the nilpotency of $L$ and $H$ it follows that $\operatorname{dim} \gamma_{i}(L)=\operatorname{dim} \gamma_{i}(H)$ for $i \geqslant 1$. Since every nilpotent Lie algebra has a homogeneous basis (see Example 2.1.2 (2)) Lemma $2.1 .10(2)$ implies that $U(L) / \omega^{k}(L)$ is finite-dimensional for all $k \geqslant 1$, and since $\operatorname{dim} \gamma_{i}(L)=\operatorname{dim} \gamma_{i}(H)$ Lemma 2.1.10 (2) again implies that

$$
\operatorname{dim} U(L) / \omega^{i}(L)=\operatorname{dim} U(H) / \omega^{i}(H),
$$

for every $i \geqslant 1$. Now, by Lemma 2.1.10 (1) $\omega(L)$ is residually nilpotent, and as $\varphi(\omega(L))=\omega(H)$, Lemma 2.1.11 implies that $\varphi$ is an isomorphism.

The following corollary says when an isomorphism in Theorem 4.2.1 is a bialgebra isomorphism. Recall $\mathcal{P}(U(L))$ denotes the set of primitive elements of $U(L)$; see discussion before Definition 1.3.7. 
Corollary 4.2.2. In Theorem 4.2.1, if $u \in \mathcal{P}(U(N))$ then $U(L) \cong U(H)$ as bialgebras.

Proof. We will show that the application $\bar{\alpha}$ in Theorem 4.2 .1 is a coalgebra homomorphism. Since the comultiplication is an algebra homomorphism it is enough verify that

$$
(\bar{\alpha} \otimes \bar{\alpha}) \Delta_{L}(z)=\Delta_{H}(\bar{\alpha}(z)),
$$

for every $z$ in a basis of $L$. Suppose that $\left\{x_{1}, \ldots, x_{n}, x\right\}$ is a basis of $L$. By the definition of $\bar{\alpha}$ we have that $(\bar{\alpha} \otimes \bar{\alpha}) \Delta_{L}\left(x_{i}\right)=\Delta_{H}\left(\bar{\alpha}\left(x_{i}\right)\right)$, for $1 \leqslant i \leqslant n$. For the element $x$ we have

$$
\begin{aligned}
(\bar{\alpha} \otimes \bar{\alpha}) \Delta_{L}(x) & =1 \otimes \bar{\alpha}(x)+\bar{\alpha}(x) \otimes 1 \\
& =1 \otimes w+w \otimes 1 \\
& =1 \otimes y+y \otimes 1-(1 \otimes u+u \otimes 1) \\
& =\Delta_{H}(y)-\Delta_{H}(u) \\
& =\Delta_{H}(w) .
\end{aligned}
$$

Therefore, $\bar{\alpha}$ is a bialgebra isomorphism.

Several of the known counterexamples for the isomorphism problem that were presented in 27 and 29] can be viewed as consequences of Theorem 4.2.1 and Corollary 4.2.2. We will illustrate this with the generic example presented in [27, Example B].

Example 4.2.3. Let $A=\left\langle x_{0}, \ldots, x_{p}\right\rangle$ be an abelian Lie algebra of dimension $p+1$ over a field $\mathbb{F}$ of characteristics $p$. Consider the Lie algebras $H=A \rtimes\langle\lambda, \pi\rangle$ and $L=A \rtimes\langle\lambda, z\rangle$ with the relations given by $\left[\lambda, x_{i}\right]=x_{i-1}$ for $1 \leqslant i \leqslant p$ and $\left[\lambda, x_{0}\right]=0$, $\left[\pi, x_{p}\right]=x_{0}$ and $\left[\pi, x_{i}\right]=0$ for $0 \leqslant i<p,[\lambda, \pi]=[z, L]=0$. Note that $L=M \oplus\langle z\rangle$ and $H=N \rtimes\langle\pi\rangle$, where $M, N$ are the ideals generated by $A$ and $\lambda$. We have that $\gamma_{n}(L)=\gamma_{n}(H)=\left\langle x_{0}, \ldots, x_{p-(n-1)}\right\rangle$ for $n \leqslant p+1$, and $\gamma_{n}(L)=\gamma_{n}(H)=0$ for $n>p+1$. Thus, it follows that $\operatorname{gr}(L) \cong \operatorname{gr}(H)$. Note that $L$ and $H$ are each metabelian and nilpotent of class $p+1$. Since char $\mathbb{F}=p$, it follows that

$$
\operatorname{ad} \lambda^{p}\left(x_{p}\right)=(\operatorname{ad} \lambda)^{p}\left(x_{p}\right)=x_{0}=\operatorname{ad} \pi\left(x_{p}\right),
$$

see equation 2.1) and ad $\lambda^{p}\left(x_{i}\right)=(\operatorname{ad} \lambda)^{p}\left(x_{i}\right)=0=\operatorname{ad} \pi\left(x_{i}\right)$ for $0 \leqslant i<p$. Hence $\left.\operatorname{ad} \lambda^{p}\right|_{N}=\left.\operatorname{ad} \pi\right|_{N}$; and by Theorem 4.2.1 we have that $U(L) \cong U(H)$. Moreover, since 
$\lambda^{p} \in \mathcal{P}(U(N))$, then by Corollary 4.2 .2 we have that $U(L) \cong U(H)$ as bialgebras.

We can use this example for verify that over the field $\mathbb{F}$ with $\operatorname{char} \mathbb{F}=2$ we have that $U\left(L_{3}\right) \cong U\left(L_{5}\right)$ as bialgebras, for the Lie algebras $L_{3}$ and $L_{5}$ presented in [29]. In fact, reordering the basis of these algebras, they can be written in the form

$$
\begin{aligned}
& L_{3}=\left\langle x_{5}, x_{4}, x_{2}, x_{1}, x_{3} \mid\left[x_{1}, x_{2}\right]=x_{4},\left[x_{1}, x_{4}\right]=x_{5}\right\rangle, \\
& L_{5}=\left\langle x_{5}, x_{4}, x_{2}, x_{1}, x_{3} \mid\left[x_{1}, x_{2}\right]=x_{4},\left[x_{1}, x_{4}\right]=x_{5},\left[x_{2}, x_{3}\right]=x_{5}\right\rangle .
\end{aligned}
$$

Now, let $A=\left\langle x_{5}, x_{4}, x_{2}\right\rangle, \lambda=x_{1}$ and $\pi=z=x_{3}$. In this way $L_{3}=A \rtimes\langle\lambda, z\rangle$ and $L_{5}=A \rtimes\langle\lambda, \pi\rangle$ with the relation as in Example 4.2 .3 with $p=2$. Thus $U\left(L_{3}\right) \cong U\left(L_{5}\right)$ as bialgebras (cf Example 2.1.6).

Other non-isomorphic nilpotent Lie algebras over the field $\mathbb{F}$ of characteristic 3 , of the paper [29], which we can apply Theorem 4.2.1 and Corollary 4.2.2, are the Lie algebras $K_{6}, K_{11}$ and $K_{7}, K_{12}$. We verify this in the following example.

Example 4.2.4. For representing these Lie algebras we assume that $\left\{x_{1}, \ldots, x_{6}\right\}$ is a basis for them, and we only write the non-zero brackets in this basis.

$$
\begin{aligned}
K_{6}:\left[x_{1}, x_{2}\right] & =x_{4},\left[x_{1}, x_{4}\right]=x_{5},\left[x_{1}, x_{5}\right]=x_{6},\left[x_{2}, x_{4}\right]=x_{6} . \\
K_{11}:\left[x_{1}, x_{2}\right] & =x_{4},\left[x_{1}, x_{4}\right]=x_{5},\left[x_{1}, x_{5}\right]=x_{6},\left[x_{2}, x_{4}\right]=x_{6},\left[x_{2}, x_{3}\right]=x_{6} . \\
K_{7}:\left[x_{1}, x_{2}\right] & =x_{4},\left[x_{1}, x_{4}\right]=x_{5},\left[x_{1}, x_{5}\right]=x_{6} . \\
K_{12}:\left[x_{1}, x_{2}\right] & =x_{4},\left[x_{1}, x_{4}\right]=x_{5},\left[x_{1}, x_{5}\right]=x_{6},\left[x_{2}, x_{3}\right]=x_{6} .
\end{aligned}
$$

First, we will see that $U\left(K_{6}\right) \cong U\left(K_{11}\right)$. Let $M$ and $N$ the subspaces generated by $\left\langle x_{1}, x_{2}, x_{4}, x_{5}, x_{6}\right\rangle$ in $K_{6}$ and $K_{11}$, respectively. In this way $K_{6}=M \oplus\left\langle x_{3}\right\rangle$ and $K_{11}=N \rtimes\left\langle x_{3}\right\rangle$. It is immediate that $M \cong N$ and that $u=-x_{1}^{3}$ verifies the hypotheses of Theorem 4.2.1. Therefore $U\left(K_{6}\right)$ and $U\left(K_{11}\right)$ are isomorphic. Moreover, since $-x_{1}^{3} \in \mathcal{P}(U(N))$, Corollary 4.2 .2 implies that $U\left(K_{6}\right) \cong U\left(K_{11}\right)$ as bialgebras.

Now, we show that $U\left(K_{7}\right) \cong U\left(K_{12}\right)$. Let $M$ and $N$ the subspaces generated by $\left\langle x_{1}, x_{2}, x_{4}, x_{5}, x_{6}\right\rangle$ in $K_{7}$ and $K_{12}$, respectively. In this way $K_{7}=M \oplus\left\langle x_{3}\right\rangle$ and $K_{12}=N \rtimes\left\langle x_{3}\right\rangle$. It is immediate that $u=-x_{1}^{3}$ verifies the hypotheses of Theorem 4.2.1. Therefore $U\left(K_{7}\right)$ and $U\left(K_{12}\right)$ are isomorphic. Moreover, since $-x_{1}^{3} \in \mathcal{P}(U(N))$, Corollary 4.2 .2 implies that $U\left(K_{7}\right) \cong U\left(K_{12}\right)$ as bialgebras. 


\subsection{Semicenter of $U(L)$}

In this section we define the semicenter of a universal enveloping algebra. We will show that in characteristic 0 this object is preserved by isomorphisms between universal enveloping algebras. So it is important to introduce this object in the study of the isomorphism problem. A more complete treatment of this topic can be found in $3,7,8$.

Let $L$ be a finite-dimensional Lie algebra over an arbitrary field $\mathbb{F}$. For each $\lambda \in L^{*}$, let

$$
U_{\lambda}(L)=\{a \in U(L) \mid[x, a]=\lambda(x) a \text { for all } x \in L\} .
$$

For $\lambda, \mu \in L^{*}$ we have that $U_{\lambda}(L) U_{\mu}(L) \subseteq U_{\lambda+\mu}(L)$. Also if $U_{\lambda}(L) \neq U_{\mu}(L)$ it is immediate to verify that $U_{\lambda}(L) \cap U_{\mu}(L)=0$. The direct sum

$$
S z(U(L))=\bigoplus_{\lambda \in L^{*}} U_{\lambda}(L)
$$

is called the semicenter of $U(L)$. Clearly $U_{0}(L)$ coincides with $Z(U(L))$, the center of $U(L)$. Suppose that $\lambda \in L^{*}$ is such that $U_{\lambda}(L) \neq 0$, then $\lambda$ is a one-dimensional representation of $L$, which implies that $\lambda\left(L^{\prime}\right)=0$. In fact, let $w \in U_{\lambda}(L) \backslash\{0\}$ then $[[x, y], w]=\lambda([x, y]) w$ for all $x, y \in L$. On the other hand

$$
\begin{aligned}
& {[[x, y], w]=-[w,[x, y]]=[x,[y, w]]+[y,[w, y]]=} \\
& \lambda(y)[x, w]-\lambda(x)[y, w]=(\lambda(x) \lambda(y)-\lambda(y) \lambda(x)) w .
\end{aligned}
$$

Therefore $\lambda([x, y])=\lambda(x) \lambda(y)-\lambda(y) \lambda(x)=0$. Thus $\lambda$ is a one-dimensional representation of $L$.

If $\lambda \in L^{*}$ is such that $U_{\lambda}(L) \neq 0$ then $\lambda(x)=0$ for every $x \in L \operatorname{such}$ that $\operatorname{ad}_{U(L)} x$ is nilpotent. In fact, let $w$ be a non-zero element of $U_{\lambda}(L)$ and suppose $\left(\operatorname{ad}_{U(L)} x\right)^{n}=0$. Then

$$
0=\left(\operatorname{ad}_{U(L)} x\right)^{n}(w)=\lambda(x)^{n} w
$$

which implies that $\lambda(x)=0$.

Example 4.3.1. Let $H=\left\langle x_{1}, \ldots, x_{n}, y_{1}, \ldots, y_{n}, z\right|\left[x_{i}, y_{i}\right]=z$ for $\left.1 \leqslant i \leqslant n\right\rangle$ be the $(2 n+1)$-dimensional Heisenberg Lie algebra over a field $\mathbb{F}$, see Example 2.1.5 (4). 
Suppose that char $\mathbb{F}=p$. Let $\lambda \in L^{*}$ such that $U_{\lambda}(L) \neq 0$. Since char $\mathbb{F}=p$, equation (2.1) and the fact that $H$ is nilpotent of class two imply that $x_{i}^{p}, y_{i}^{p} \in Z(U(L))$. Thus $\operatorname{ad}_{U(L)} x_{i}$ and $\operatorname{ad}_{U(L)} y_{i}$ are nilpotent. Hence $\lambda\left(x_{i}\right)=\lambda\left(y_{i}\right)=0$, and since $H^{\prime}=\langle z\rangle$ it follows that $\lambda(z)=0$. Therefore $\lambda=0$, and this implies that

$$
S z(U(L))=U_{0}(L)=Z(U(L)) .
$$

In [8, Proposition 4.3.5] Dixmier showed that if $\operatorname{char} \mathbb{F}=0$ then $S z(U(L))$ is a commutative subalgebra of $U(L)$. Later, in [3, Proposition 2.1] Braun and Vernik showed that this is true in any characteristic. We will show that in characteristic 0 the semicenter is preserved by isomorphisms between universal enveloping algebras. Before this, we need to introduce another notation. We recall that $Q(L)$ denotes the ring of quotients of $U(L)$, see Section 2.2. For $\lambda \in L^{*}$, let

$$
Q_{\lambda}(L)=\{a \in Q(L) \mid[x, a]=\lambda(x) a \text { for all } x \in L\}
$$

Clearly $U_{\lambda}(L)=Q_{\lambda}(L) \cap U(L)$.

Lemma 4.3.2. Let $L$ be a finite-dimensional Lie algebra over a field $\mathbb{F}$ of characteristic 0 , and $a \in Q(L), a \neq 0$. Then, $a^{-1} U(L) a=U(L)$ if and only if there exists $\lambda \in L^{*}$ such that $a \in Q_{\lambda}(L)$.

Proof. The proof follows applying [18, Theorem 1] with $P=0$.

Theorem 4.3.3. Let $L, H$ be finite-dimensional Lie algebras over a field $\mathbb{F}$ of characteristic 0, and suppose that $\varphi: U(L) \rightarrow U(H)$ is an algebra isomorphism. Then $\varphi(S z(U(L)))=S z(U(H))$. Consequently, $S z(U(L))$ is invariant under Aut $(U(L))$.

Proof. Suppose that $\varphi: U(L) \rightarrow U(H)$ is an algebra isomorphism. This isomorphism induces an isomorphism $\bar{\varphi}: Q(L) \rightarrow Q(H)$, defined by $\bar{\varphi}\left(a s^{-1}\right)=\varphi(a) \varphi(s)^{-1}$. Given $\lambda \in L^{*}$, let $a$ be a nonzero element in $U_{\lambda}(L)$. Then, by Lemma 4.3.2, $a^{-1} U(L) a=$ $U(L)$. Applying $\bar{\varphi}$ we have that $\varphi(a)^{-1} U(H) \varphi(a)=U(H)$. Lemma 4.3.2 again implies that $\varphi(a) \in Q_{\mu}(H)$, for some $\mu \in H^{*}$. However, since $\varphi(a) \in U(H)$ it follows that $\varphi(a) \in Q_{\mu}(H) \cap U(H)=U_{\mu}(H)$. This implies that $\varphi(S z(U(L))) \subseteq S z(U(H))$. Using the same argument for $\varphi^{-1}$ we obtain that $\varphi^{-1}(S z(U(H))) \subseteq S z(U(L))$. Therefore, $\varphi(S z(U(L)))=S z(U(H))$. Consequently, if $\varphi: U(L) \rightarrow U(L)$ is an automorphism of $U(L)$, then we have that $\varphi(S z(U(L)))=S z(U(L))$. 


\subsection{The Frobenius Semiradical of a Lie algebra}

Let $L$ be a finite-dimensional Lie algebra over an arbitrary field $\mathbb{F}$. Let $f \in L^{*}$, and define an alternating bilinear form $B_{f}: L \times L \rightarrow \mathbb{F}$, by $B_{f}(x, y)=f([x, y])$. The radical of this bilinear form is the set

$$
L(f)=\{x \in L \mid f([x, y])=0 \text { for all } y \in L\} .
$$

It is easy to see that $L(f)$ is a Lie subalgebra (which may not be an ideal) of $L$ containing the center $Z(L)$ of $L$. Let $f \in L^{*}$. Then the bilinear form $B_{f}$ induces a non-degenerate alternating bilinear form on $L / L(f)$, defined by $(x+L(f), y+L(f)) \mapsto$ $B_{f}(x, y)$. Since the dimension of a vector space with a non-degenerate alternating bilinear form is even, we have that $\operatorname{dim} L-\operatorname{dim} L(f)$ is an even number.

Set

$$
i(L)=\min _{f \in L^{*}} \operatorname{dim} L(f) ;
$$

the number $i(L)$ is referred to as the index of $L$. In particular we have that $\operatorname{dim} L-i(L)$ is even. Moreover, $i(L)=\operatorname{dim} L$ if and only if $L$ is abelian. An element $f \in L^{*}$ is called regular if $\operatorname{dim} L(f)=i(L)$. The set of regular elements is denoted by $L_{\text {reg }}^{*}$.

The set

$$
F(L)=\sum_{f \in L_{\text {reg }}^{*}} L(f)
$$

is called the Frobenius semiradical of $L$. This is a subalgebra of $L$. Let $\alpha \in \operatorname{Aut}(L)$; then $\alpha(L(f))=L\left(f \circ \alpha^{-1}\right)$ for all $f \in L^{*}$. Hence $\alpha(F(L))=F(L)$, and it follows that $F(L)$ is a characteristic subalgebra of $L$ containing $Z(L)$. Note that if $F(L) \neq L$, then $\left.f\right|_{L^{\prime}} \neq 0$ for every regular element $f$. In fact, if $\left.f\right|_{L^{\prime}}=0$ for some $f \in L_{\text {reg }}^{*}$, then $L(f)=\{x \in L \mid f([x, y])=0$ for all $y \in L\}=L$, which implies that $F(L)=L$. On other hand, $F(L)=0$ if and only if there exists $f \in L^{*}$ such that the bilinear form $B_{f}$ is non-degenerated. The last is equivalent to that the matrix of the bilinear form $B_{f}$, in some base of $L$, is non-singular.

Example 4.4.1. Let $L=\left\langle x_{1}, \ldots, x_{6} \mid\left[x_{1}, x_{2}\right]=x_{5},\left[x_{1}, x_{3}\right]=x_{6},\left[x_{2}, x_{4}\right]=x_{6}\right\rangle$. A calculation shows that $Z(L)=\left\langle x_{5}, x_{6}\right\rangle$. Let $f \in L^{*}$, and let $x=\alpha_{1} x_{1}+\cdots+\alpha_{6} x_{6}$ an 
element in $L(f)$. Then

$$
\begin{aligned}
& 0=f\left(\left[x, x_{2}\right]\right)=\alpha_{1} f\left(x_{5}\right)-\alpha_{4} f\left(x_{6}\right), \\
& 0=f\left(\left[x, x_{3}\right]\right)=\alpha_{1} f\left(x_{6}\right), \\
& 0=f\left(\left[x, x_{1}\right]\right)=-\alpha_{2} f\left(x_{5}\right)-\alpha_{3} f\left(x_{6}\right), \\
& 0=f\left(\left[x, x_{4}\right]\right)=\alpha_{2} f\left(x_{6}\right) .
\end{aligned}
$$

Let $\left\{\varphi_{1}, \ldots, \varphi_{6}\right\}$ be the dual basis of $\left\{x_{1}, \ldots, x_{6}\right\}$ and consider the linear functional $f=$ $\varphi_{5}+\varphi_{6}$. If $x \in L(f)$ as above, then $\alpha_{i}=0$ for $i=1, \ldots, 4$; and consequently $L(f) \subseteq$ $Z(L)$. Since $L(f)$ contains $Z(L)$, we obtain that $L(f)=Z(L)$. Therefore $i(L)=2$, and for all $f \in L_{\mathrm{reg}}^{*}$ we have that $L(f)=Z(L)$. Hence $F(L)=\sum_{f \in L_{\mathrm{reg}}^{*}} L(f)=Z(L)$.

Lemma 4.4.2. Suppose that $L$ is a finite-dimensional Lie algebra of dimension at least 3 over a field $\mathbb{F}$ and that $H$ is an abelian subalgebra of codimension 1 of L. Let $f \in L^{*}$. Then the bilinear form $B_{f}:(x, y) \mapsto f([x, y])$ is degenerate. Consequently, $F(L) \neq 0$.

Proof. Suppose that $\left\{x_{1}, \ldots, x_{n}\right\}$ is a basis of $H$ and complete this basis to a basis $\left\{x_{1}, \ldots, x_{n}, x_{n+1}\right\}$ of $L$. Since $H$ is abelian, the matrix of $B_{f}$ with respect to the basis $\left\{x_{1}, \ldots, x_{n}, x_{n+1}\right\}$ is an anti-symmetric matrix of the form

$$
\left(\begin{array}{ccccc}
0 & 0 & \cdots & 0 & * \\
0 & 0 & \cdots & 0 & * \\
\vdots & \vdots & \ddots & \vdots & * \\
0 & 0 & \cdots & 0 & * \\
* & * & \cdots & * & 0
\end{array}\right) .
$$

Clearly this matrix is singular, and it follows that $B_{f}$ is degenerate. Thus $L(f) \neq 0$, and since $f$ is arbitrary we have that $F(L) \neq 0$.

A Lie algebra $L$ is called square integrable if $Z(L)=F(L)$. Clearly this is equivalent to $\operatorname{dim} Z(L)=i(L)$. For instance, the Lie algebra $L$ of Example 4.4.1 is square integrable. We will also see later, that some Lie algebras of Group 5, in the group division in Section 3.1, are square integrable. The following lemma guarantees that there exist many examples of square integrable Lie algebras. 
Lemma 4.4.3. Let $L$ be a finite-dimensional Lie algebra over a field $\mathbb{F}$. Suppose that $\operatorname{dim} L^{\prime}=1$. Then, $L$ is square integrable.

Proof. Suppose that $L^{\prime}=\langle z\rangle$, and let $\varphi$ be a linear functional of $L$ such that $\varphi(z)=1$. Let $x \in L(\varphi)$. Then $\varphi([x, y])=0$ for each $y \in L$. Since $L^{\prime}=\langle z\rangle$, for each $y \in L$ there exists $\alpha_{y} \in \mathbb{F}$ such that $[x, y]=\alpha_{y} z$. Thus

$$
\alpha_{y}=\alpha_{y} \varphi(z)=\varphi\left(\alpha_{y} z\right)=\varphi([x, y])=0
$$

for each $y \in L$, and it follows that $x \in Z(L)$; and so $L(\varphi) \subseteq Z(L)$. On the other hand, it is clear that $Z(L) \subseteq L(\varphi)$, consequently $L(\varphi)=Z(L)$. Thus, for each $f \in L_{\text {reg }}^{*}$ we have $L(f)=Z(L)$. Hence $F(L)=\sum_{f \in L_{\text {reg }}^{*}} f(L)=Z(L)$.

The following lemma relates the Frobenius semiradical with the center of the universal enveloping algebra. This relation is very useful for deciding if two universal enveloping algebras are isomorphic or not. This lemma also is very useful for the calculation of the Frobenius semiradical.

Lemma 4.4.4. Let $L$ be a finite-dimensional Lie algebra over a field $\mathbb{F}$ of characteristic 0. Then the following are valid.

1. Let $M$ be a Lie subalgebra of $L$ of codimension one. Then $i(M)=i(L)+1$ if and only if $F(L) \subseteq M$.

2. If $u \in L$ is such that $C_{L}(u)$ has codimension one in $L$, then $i\left(C_{L}(u)\right)=i(L)+1$.

3. $Z(U(L)) \subseteq U(F(L))$.

Proof. See [9, Proposition 1.6 (4)] for part (1), 9, Proposition 1.9 (1)] for part (2) and [21, Theorem $2.5(1)]$ for part (3).

Part (3) of Lemma 4.4 .4 gives a necessary condition for an element to be central in $U(L)$. In general, the containment stated in Lemma 4.4.4 (3) is not valid in characteristic $p$. We see this in the following example. 
Example 4.4.5. Let $L=\left\langle x_{1}, \ldots, x_{6} \mid\left[x_{1}, x_{2}\right]=x_{5},\left[x_{1}, x_{3}\right]=x_{6},\left[x_{2}, x_{4}\right]=x_{6}\right\rangle$. In Example 4.4.1 we saw that $F(L)=Z(L)=\left\langle x_{5}, x_{6}\right\rangle$. Therefore $U(F(L))=\mathbb{F}\left[x_{5}, x_{6}\right]$. If char $\mathbb{F}=p$, then equation (2.1) and the fact that $L$ is nilpotent of class 2 imply that $0=\left(\operatorname{ad} x_{1}\right)^{p}=\operatorname{ad}\left(x_{1}^{p}\right)$. Thus $x_{1}^{p} \in Z(U(L))$, but it is not true that $x_{1}^{p} \in U(F(L))$. Hence $Z(U(L)) \nsubseteq U(F(L))$.

Using Lemma 4.4.4 (3) we can show an interesting property of square integrable Lie algebras over a field $\mathbb{F}$ of characteristic 0 . Suppose that $L$ is a square integrable Lie algebra over a field $\mathbb{F}$ of characteristic 0. Then Lemma 4.4.4 (3) implies that $Z(U(L)) \subseteq U(Z(L))$. On other hand, it is clear that $U(Z(L)) \subseteq Z(U(L))$. Thus, $Z(U(L))=U(Z(L))$. That is, the associative subalgebra of $U(L)$ generated by the center of $L$ is equal to the center of $U(L)$. Thus we have shown the following theorem.

Theorem 4.4.6. Suppose that $L$ is a square integrable Lie algebra over a field $\mathbb{F}$ of characteristic 0. Then $U(Z(L))=Z(U(L))$.

In the following example we calculate the center of the universal enveloping algebra of the Heisenberg Lie algebra over a field of characteristic zero. It is well known that this center is the associative subalgebra generated by the center of the Heisenberg Lie algebra. We will obtain this with the results shown so far.

Example 4.4.7. Let $H=\left\langle x_{1}, \ldots, x_{n}, y_{1}, \ldots, y_{n}, z\right|\left[x_{i}, y_{i}\right]=z$ for $\left.1 \leqslant i \leqslant n\right\rangle$ be the $(2 n+1)$-dimensional Heisenberg Lie algebra over a field $\mathbb{F}$, see Example 2.1.5 (4). Then $H^{\prime}=Z(H)=\langle z\rangle$. By Lemma 4.4.3 we have that $H$ is square integrable. Moreover, if $\operatorname{char} \mathbb{F}=0$, then Theorem 4.4.6 implies that $Z(U(H))=U(Z(H))=\mathbb{F}[z]$ is the polynomial ring in the indeterminate $z$.

Usefi in [32, Section 8] gave a list of open problems and the following question can be found:

Let $L$ be a finite-dimensional Lie algebra over a field of characteristic zero. Is $Z(L)$ determined by $U(L)$ ?

In general we do not know if this is true for any Lie algebra, but in the following theorem we show that in the class of finite-dimensional square integrable Lie algebras this question has a positive answer. 
Theorem 4.4.8. Suppose that $L$ and $H$ are finite-dimensional square integrable Lie algebras over a field $\mathbb{F}$ of characteristic 0 , and that $\varphi: U(L) \rightarrow U(H)$ is an algebra isomorphism. Then, $\operatorname{dim} Z(L)=\operatorname{dim} Z(H)$.

Proof. Since $L$ and $H$ are square integrable, Theorem 4.4 .6 implies that $Z(U(L))=$ $U(Z(L))$ and $Z(U(H))=U(Z(H))$. Using the second equality and applying $\varphi$ we have that

$$
U(Z(H))=Z(U(H))=\varphi(Z(U(L)))=\varphi(U(Z(L))) .
$$

Therefore, $\left.\varphi\right|_{U(Z(L))}$ is an isomorphism between $U(Z(L))$ and $U(Z(H))$. Thus, by Lemma 2.3.3 (1) it follows that $\operatorname{dim} Z(L)=\operatorname{dim} Z(H)$.

We will make a brief study of the square integrable Lie algebras in dimension 4 , and as a consequence, we obtain that many Lie algebras of the group division in Section 3.1 are square integrable.

Lemma 4.4.9. Let $L$ be a Lie algebra of dimension 4 over an arbitrary field $\mathbb{F}$. The following holds.

1. If $L$ is square integrable and $Z(L) \neq 0$, then $L$ is abelian or $\operatorname{dim} Z(L)=2$.

2. If $L$ is abelian or $\operatorname{dim} Z(L)=2$, then $L$ is square integrable.

Proof. (1) Since $\operatorname{dim} L-i(L)$ is an even number, it follows that $i(L)=0,2$ or 4 . Since $Z(L) \neq 0$, we have that $i(L) \neq 0$. If $i(L)=2$, then $\operatorname{dim} Z(L)=i(L)=2$, since $L$ is square integrable. Now, if $i(L)=4$ it follows that $Z(L)=L$ and thus $L$ is abelian.

(2) If $L$ is abelian, then it is clear that $F(L)=Z(L)$. Suppose that $\operatorname{dim} Z(L)=2$, since $\operatorname{dim} L-i(L)$ is even and $i(L)<4$, we have that $i(L)=2$. This implies that $F(L)=Z(L)$.

We are interested in knowing which of the algebras in Section 3.1 are square integrable. By Lemma 4.4.9 (2) the algebras $M^{1}, M^{4}$ and $M^{5}$ are square integrable Lie algebras, and by Lemma 4.4.9 (1) it is enough search square integrable Lie algebras among the Lie algebras which have trivial center. By Lemma 4.4 .2 we have that the Lie algebras in Group 4, of the group division in Section 3.1, are not square integrable. Only remains to know if the Lie algebras with trivial center that do not belong to 
Group 4, in the group division in Section 3.1, are square integrable. For this, observe that if $L$ is a Lie algebra in Section 3.1 with basis $\left\{x_{1}, x_{2}, x_{3}, x_{4}\right\}$ and trivial center and different from $M^{8}$, then the subalgebra generated by $\left\langle x_{1}, x_{2}\right\rangle$ is abelian. In the case of $M^{8}$, we have that the subalgebra generated by $\left\langle x_{1}, x_{4}\right\rangle$ is abelian. We will consider a basis $\left\{y_{1}, y_{2}, y_{3}, y_{4}\right\}$ for these Lie algebras where $y_{i}=x_{i}$ for the Lie algebras different from $M^{8}$ and $y_{1}=x_{1}, y_{2}=x_{4}, y_{3}=x_{3}, y_{4}=x_{1}$ for $M^{8}$. In this way in all Lie algebras we have that the subalgebra $\left\langle y_{1}, y_{2}\right\rangle$ is abelian. We will denote by $\left\{\varphi_{1}, \varphi_{2}, \varphi_{3}, \varphi_{4}\right\}$ the dual basis of $\left\{y_{1}, y_{2}, y_{3}, y_{4}\right\}$. Now, let $f \in L^{*}$ and define the bilinear form $B_{f}: L \times L \rightarrow \mathbb{F}$ by $B_{f}(x, y)=f([x, y])$, as Section 4.4. Since $\left\langle y_{1}, y_{2}\right\rangle$ is abelian, the matrix of $B_{f}$ in the basis $\left\{y_{1}, y_{2}, y_{3}, y_{4}\right\}$ has the form

$$
\left(\begin{array}{c|c}
0 & -A^{T} \\
\hline A & *
\end{array}\right)
$$

where

$$
A=\left(\begin{array}{ll}
f\left(\left[y_{3}, y_{1}\right]\right) & f\left(\left[y_{3}, y_{2}\right]\right) \\
f\left(\left[y_{4}, y_{1}\right]\right) & f\left(\left[y_{4}, y_{2}\right]\right)
\end{array}\right)
$$

The bilinear form $B_{f}$ is non-degenerate if and only if $\operatorname{det}\left(A A^{T}\right) \neq 0$. Therefore, to show that the Frobenius semiradical of these algebras is zero it is enough find a linear functional $f$ such that $\operatorname{det} A \neq 0$, where $A$ is as above.

In the following table we display the information that will be necessary to show that some Lie algebras in Section 3.1, which have a trivial center, are square integrable Lie algebras. The first column contains the name of the Lie algebra with the notation of Section 3.1. The second column contains a linear functional $f$ and the third column contains the matrix $A$ in the basis $\left\{y_{1}, y_{2}, y_{3}, y_{4}\right\}$, defined as above. The matrix $A$ is obtained by observing the multiplication table of the respective Lie algebras and applying the functional $f$. 


\begin{tabular}{|c|c|c|}
\hline Name & functional & matrix \\
\hline$M^{12}$ & $\varphi_{2}$ & $\left(\begin{array}{ll}1 & 0 \\
0 & 2\end{array}\right)$ \\
\hline$M_{a}^{13}$ & $\varphi_{2}$ & $\left(\begin{array}{ll}1 & 0 \\
0 & 1\end{array}\right)$ \\
\hline$M_{a, b}^{11}$ & $\varphi_{1}$ & $\left(\begin{array}{ll}0 & a \\
1 & 0\end{array}\right)$ \\
\hline$M^{8}$ & $\varphi_{1}+\varphi_{2}$ & $\left(\begin{array}{ll}0 & 1 \\
-1 & 0\end{array}\right)$ \\
\hline$M_{a}^{9}$ & $\varphi_{1}$ & $\left(\begin{array}{ll}1 & 0 \\
0 & 1\end{array}\right)$ \\
\hline$M_{0}^{13}$ & $\varphi_{2}$ & $\left(\begin{array}{ll}1 & 0 \\
0 & 1\end{array}\right)$ \\
\hline$M_{a}^{10}$ & $\varphi_{2}$ & $\left(\begin{array}{ll}0 & 1 \\
1 & 0\end{array}\right)$ \\
\hline
\end{tabular}

Theorem 4.4.10. With the notation of Section 3.1, we have that the solvable square integrable Lie algebras of dimension 4 are precisely

$$
M^{1}, M^{4}, M^{5}, M^{8}, M_{a}^{9}, M_{a}^{10}, M_{a, b}^{11}, M^{12} \text { if char } \mathbb{F} \neq 2, M_{a}^{13}
$$

Proof. By Lemma 4.4.9 (2) we have that $M^{1}, M^{4}, M^{5}$ are square integrable Lie algebras; and by Lemma 4.4 .9 (1) it is enough search square integrable Lie algebras among the Lie algebras which have trivial center. Now, Lemma 4.4 .2 implies that the Lie algebras in Group 4, of the group division in Section 3.1, are not square integrable. Thus, the only possibilities for square integrable Lie algebras are those that are in the table above. Since the matrices in the third column of this table are non-singular, the Frobenius semiradical of these algebras is zero (note that here we use that char $\mathbb{F} \neq 2$ in the case of $M^{12}$ ). Therefore these Lie algebras are square integrable. 
An immediate consequence of Theorem 4.4.10 and Theorem 4.4.6 is the following corollary.

Corollary 4.4.11. Let $L$ be one of the Lie algebras in Theorem 4.4.10 over a field of characteristic 0. Then $Z(U(L))$ is a polynomial ring with $\operatorname{dim} Z(L)$ indeterminates.

We conclude this section with a proposition which is a partial result towards the second step of the proof of Theorem 3.2.1. But before we need to subdivide of Group 5 , of the group division in Section 3.1, into two subgroups. This subdivision separates the Lie algebras of Group 5, which are square integrable from those that are not. We follow the notation in Section 3.1 and refer to the same section for all information about these algebras. The subgroups of Group 5 are the following

Group 5.1 (not square integrable): $M_{0}^{3}, M_{0, b}^{6}, M_{0, b}^{7}$.

Group 5.2 (square integrable): $M^{8}, M_{a}^{9}, M_{a}^{13}, M^{10}$.

Proposition 4.4.12. Suppose that $\mathbb{F}$ is a field of characteristic 0 . If $L$ belongs to Group 5.1 and $H$ belongs to Group 5.2 in the subdivision above, then $U(L) \not U(H)$.

Proof. In Theorem 4.4.10 we showed that $F(H)=0$. Thus, Lemma 4.4 .4 (3) implies that $Z(U(H)) \subseteq U(0)=\mathbb{F}$, and hence $Z(U(H))=\mathbb{F}$. On other hand, we have that $Z(L) \neq 0$, see the table of Group 5 in Section 3.1. This implies that $Z(U(L)) \neq \mathbb{F}$. Hence $U(L) \not U(H)$. 


\section{Chapter 5}

\section{Universal enveloping algebras of certain Lie algebras}

In Chapter 2, after Lemma 2.3.3, we saw that the isomorphism problem has a positive solution for finite-dimensional abelian Lie algebras. A natural way to continue addressing the isomorphism problem is to ask: What happens when we consider Lie algebras having an abelian ideal of codimension 1? Will the isomorphism problem have a positive solution? Despite addressing this in the next chapter, the motivation for this chapter was to search for an answer for this question. In general, in this chapter we consider the question above with a proper abelian ideal and we will see that the answer for this question is positive under some conditions.

This chapter consists of two sections. In Section 5.1, given a Lie algebra $L$ and an abelian ideal $M$, we introduce a new Lie algebra $L_{M}$ which is invariant under a certain group of automorphisms of $U(L)$, and we will show that in some cases $L_{M}$ is isomorphic to $L$. This section was inspired by an analysis of the paper by Chun, Kajiwara and Lee [4]. Section 5.2 is dedicated to the calculation of the algebra $L_{M}$ for different ideals $M$. With these calculations we give more examples of Lie algebras with non-isomorphic universal enveloping algebras.

\subsection{The algebra $L_{M}$}

The aim of this section is to present a construction that, for a Lie algebra $L$ and an abelian ideal $M$ of $L$, outputs another Lie algebra $L_{M}$ which is invariant under 
a certain group of automorphisms of $U(L)$. We will show that $L_{M}$ is sometimes isomorphic to $L$, and in such cases the isomorphism type of $L$ is determined by the isomorphism type of $U(L)$.

Throughout this section, $L$ denotes a finite-dimensional Lie algebra over an arbitrary field $\mathbb{F}, M$ an abelian ideal of $L$ and $K$ denotes the quotient $L / M$. Since $M$ is abelian, the adjoint representation $\operatorname{ad}_{K, M}: K \rightarrow \mathfrak{g l}(M)$ of $K$ on $M$, given by $\operatorname{ad}_{K, M}(k+M)(a)=[k, a]$ for all $k+M \in K$ and $a \in M$, is well-defined. Then the image $\operatorname{ad}_{K, M}(K)$ of $K$ under $\operatorname{ad}_{K, M}$ is a subalgebra of $\mathfrak{g l}(M)$. Also, Lemma 2.3.1 (1) implies that $M U(L)$ is a two-sided ideal of $U(L)$ and by Lemma 2.3.1 (2) we have $U(K) \cong U(L) / M U(L)$.

Let $\mathfrak{m}$ be a maximal two-sided ideal of $U(L)$ such that $U(L) / \mathfrak{m} \cong \mathbb{F}$ and $M U(L) \subseteq$ $\mathfrak{m}$. It is clear that $M U(L) \mathfrak{m}=M \mathfrak{m}$. Set

$$
\widetilde{I}=M U(L) / M \mathfrak{m}
$$

and consider $\tilde{I}$ as an abelian Lie algebra. Since $M$ is abelian, by [4, Proposition 2.4], the map

$$
\vartheta: x \mapsto x+M \mathfrak{m}
$$

is an isomorphism of the (abelian) Lie algebras $M$ and $\widetilde{I}$. Consequently, if $\left\{x_{i}\right\}_{i \in \mathcal{I}}$ is a basis of $M$ then $\left\{x_{i}+M \mathfrak{m}\right\}_{i \in \mathcal{I}}$ is a basis of $\widetilde{I}$.

Define a map $\beta: U(L) / M U(L) \rightarrow \mathfrak{g l}(\widetilde{I})$ by setting

$$
\beta(w+M U(L))(a+M \mathfrak{m})=[w, a]+M \mathfrak{m}=(w a-a w)+M \mathfrak{m}
$$

for all $w \in U(L)$ and $a \in M U(L)$. We claim that $\beta$ is a well-defined homomorphism of Lie algebras. In fact, suppose that $w \in U(L), a, v \in M U(L), u \in M \mathfrak{m}$; and note that $M U(L) \subseteq \mathfrak{m}$. Then

$$
[w+v, a+u]=[w, a]+[w, u]+[v, a]+[v, u]
$$

Now $[w, u]=w u-u w \in M \mathfrak{m},[v, a]=v a-a v \in M \mathfrak{m}$ since the Lie algebra $\tilde{I}$ is abelian, and $[v, u]=v u-u v \in M \mathfrak{m}$. Thus $[w+v, a+u]+M \mathfrak{m}=[w, a]+M \mathfrak{m}$. Since $\beta$ is induced by the adjoint action of $U(L)$ on the ideal $M U(L)$, we have that $\beta: U(L) / M U(L) \rightarrow \mathfrak{g l}(\widetilde{I})$ is a Lie homomorphism. 
Let $J$ be the unique Lie ideal of $U(L)$ that contains $M U(L)$ and such that $\operatorname{ker} \beta=$ $J / M U(L)$. That is

$$
J=\{w \in U(L) \mid[w, a] \in M \mathfrak{m} \text { for all } a \in M U(L)\} .
$$

Recall that the semidirect product of two Lie algebras is defined before Lemma 2.1.3. Consider $\widetilde{I}$ and $\widetilde{K}=U(L) / J$ as Lie algebras and set

$$
L_{M}=\widetilde{I} \rtimes_{\beta} \widetilde{K} .
$$

Then $L_{M}$ is a Lie algebra. In the following proposition we will investigate the principal properties of the algebra $L_{M}$ and the ideal $J$.

Proposition 5.1.1. Let $L$ be a finite-dimensional Lie algebra and $M$ an abelian ideal of $L$. Then the following are valid.

1. $L_{M}$ is a finite-dimensional Lie algebra. Moreover, $L_{M}$ is metabelian if $K$ is abelian.

2. $J \cap \mathfrak{m}$ is a two-sided associative ideal of $U(L)$.

3. Suppose that $\mathfrak{m}=\omega(L)$. Then $L \cap J=C_{L}(M)$ and $C_{L}(M) U(L) \subseteq J$.

4. Suppose that $\mathfrak{m}=\omega(L)$. Then there exists an injective homomorphism $\tilde{\chi}$ from $M \rtimes L / C_{L}(M)$ to $L_{M}$ which is an isomorphism if and only if $\operatorname{dim} \frac{L}{C_{L}(M)}=\operatorname{dim} \widetilde{K}$.

Proof. (1) Since $\beta$ is a Lie algebra homomorphism, it follows that $L_{M}$ is a Lie algebra. Note that $\beta$ induces an injective homomorphism $\widetilde{K} \rightarrow \mathfrak{g l}(\widetilde{I})$ and so $\widetilde{K}$ is finite-dimensional. Hence $L_{M}$ is also finite-dimensional. Now, if $K=L / M$ is abelian then also is $U(K)=U(L) / M U(L)$, and consequently so is $\widetilde{K}$. Thus, since $L_{M}$ is defined as a semidirect sum of two abelian Lie algebras, $L_{M}$ is metabelian.

(2) Note that $J \cap \mathfrak{m}$ is a Lie ideal of $U(L)$. Therefore, in order to show that $J \cap \mathfrak{m}$ is a two-sided associative ideal, it is enough show that $J \cap \mathfrak{m}$ is a right ideal. Suppose that $w \in J \cap \mathfrak{m}$ and let $u \in U(L)$. Then, for $a \in M U(L)$, we have that

$$
[w u, a]=[w, a] u+w[u, a]=[w, a] u+[w,[u, a]]+[u, a] w .
$$


Since $w \in J$ the first two summands are in $M \mathfrak{m}$, and since $w \in \mathfrak{m}$ the last summand is in $M \mathfrak{m}$. Thus $w u \in J \cap \mathfrak{m}$.

(3) Suppose $x \in L \cap J$. Then, by definition of $J$ (see equation (5.3)), for all $y \in M$ we have

$$
[x, y] \in L \cap M \mathfrak{m}=L \cap M \omega(L)=M^{\prime}=0,
$$

see Lemma 2.3.1 (4) for the second equality. Thus $x \in C_{L}(M)$, and the inclusion $L \cap J \subseteq C_{L}(M)$ holds. For the converse inclusion, let $x \in C_{L}(M)$ and let $a=\sum_{i=1}^{n} x_{i} u_{i}$; where $x_{i} \in M$ and $u_{i} \in U(L)$; be an arbitrary element of $M U(L)$. We will show that $[x, a] \in M \mathfrak{m}$, and thus $x \in J$. Since $\left[x, x_{i}\right]=0$ for all $i$, it follows that

$$
[x, a]=x \sum_{i=1}^{n} x_{i} u_{i}-\sum_{i=1}^{n} x_{i} u_{i} x=\sum_{i=1}^{n} x_{i} x u_{i}-\sum_{i=1}^{n} x_{i} u_{i} x .
$$

Since every summand of the right-hand side of the last equation lies in $M \mathfrak{m}$ it follows that $x \in L \cap J$. Thus $C_{L}(M) \subseteq L \cap J$, and the equality $J \cap L=C_{L}(M)$ follows. For the second affirmation note that $C_{L}(M)$ is an ideal of $L$, and so $C_{L}(M) U(L)$ is a two-sided ideal of $U(L)$, by Lemma 2.3.1 (1). As $J \cap \mathfrak{m}$ is a two-sided ideal of $U(L)$ which contains $C_{L}(M)$, it follows that $C_{L}(M) U(L) \subseteq J \cap \mathfrak{m} \subseteq J$.

(4) We have that $U(K) \cong U(L) / M U(L)$ under the isomorphism $k \mapsto k+M U(L)$ for each $k \in K$. Composing this isomorphism with the natural homomorphism $U(L) / M U(L) \rightarrow U(L) / J$ we obtain an homomorphism $\iota: K \rightarrow \widetilde{K}$, defined by $\iota(k)=k+J$. On the other hand, the isomorphism $\vartheta: M \rightarrow \widetilde{I}$ induces an isomorphism $\vartheta^{*}: \mathfrak{g l}(M) \rightarrow \mathfrak{g l}(\widetilde{I})$, given by $\alpha \mapsto \vartheta \alpha \vartheta^{-1}$. We claim that the following diagram commutes

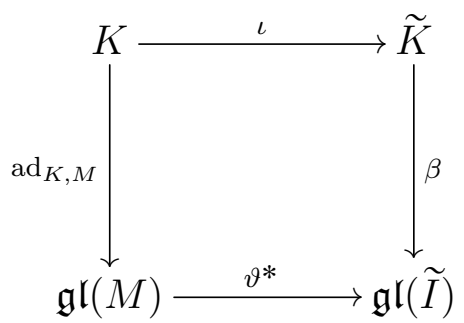

Suppose that $k \in K$ and $a+M \mathfrak{m} \in \widetilde{I}$. As noted after of (5.1), the elements $x_{i}+M \mathfrak{m}$ form a basis for $\widetilde{I}$, where the set $\left\{x_{i}\right\}_{i}$ is a basis for $M$. Hence we may assume without 
loss of generality that $a \in M$. Then

$$
\begin{aligned}
\beta(k+M U(L))(a+M \mathfrak{m})=[ & {[k, a]+M \mathfrak{m}=\operatorname{ad}_{K, M}(k)(a)+M \mathfrak{m}=} \\
& \vartheta \operatorname{ad}_{K, M}(k) \vartheta^{-1}(a+M \mathfrak{m})=\vartheta^{*}\left(\operatorname{ad}_{K, M}(k)\right)(a+M \mathfrak{m}) .
\end{aligned}
$$

Thus, the diagram commutes. By Lemma 2.1.3 the map $\chi: M \rtimes K \rightarrow L_{M}=\widetilde{I} \rtimes_{\beta} \tilde{K}$, defined by $\chi(a, k)=(\vartheta(a), \iota(k))$, is a homomorphism between the Lie algebras $M \rtimes K$ and $L_{M}$. Now, an element $x+M$ of $K$ is in the kernel of $\iota$ if and only if $\iota(x)=J$, if and only if $x \in J \cap L=C_{L}(M)$, see item (3). Therefore ker $\iota=C_{L}(M) / M$, and thus $\iota$ induces an injective homomorphism between $K / \operatorname{ker} \iota$ and $\widetilde{K}$. On the other hand, it is clear that $\operatorname{ker}_{K, M}=C_{L}(M) / M$. Thus, $\operatorname{ad}_{K, M}$ induces an injective homomorphism between $K / \operatorname{ker}\left(\operatorname{ad}_{K, M}\right)$ and $\mathfrak{g l}(M)$. Note that $K / \operatorname{ker} \iota=K / \operatorname{ker}\left(\operatorname{ad}_{K, M}\right)=L / C_{L}(M)$. Hence, we have the following commutative diagram

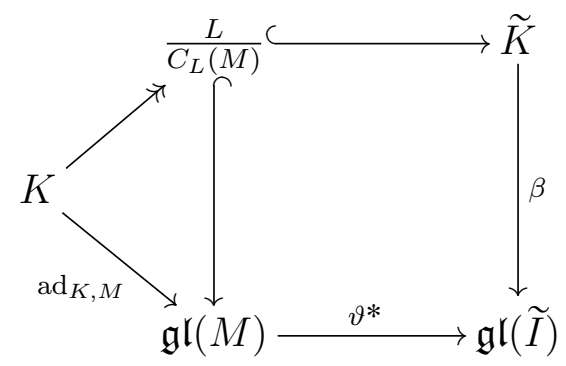

Thus, $\chi$ induces an injective homomorphism $\tilde{\chi}: M \rtimes \frac{L}{C_{L}(M)} \rightarrow L_{M}=\widetilde{I} \rtimes \widetilde{K}$, which is defined as $\tilde{\chi}\left(a, x+C_{L}(M)\right)=(a+M \mathfrak{m}, x+J)$. It is immediate that $\tilde{\chi}$ is an isomorphsim if and only if $\operatorname{dim} \frac{L}{C_{L}(M)}=\operatorname{dim} \tilde{K}$.

In the next result we use $\lfloor t\rfloor$ for the largest integer less than or equal to $t$.

Corollary 5.1.2. Let $L$ be a Lie algebra and $M$ an abelian ideal of $L$ containing $L^{\prime}$. Suppose that $L=M \rtimes K$, where $\operatorname{dim} K \geqslant\left\lfloor\frac{(\operatorname{dim} M)^{2}}{4}\right\rfloor+1$. Then, choosing $\mathfrak{m}=\omega(L)$, we have that:

1. $L_{M} \cong M \rtimes \frac{L}{C_{L}(M)}$.

2. If $C_{L}(M)=M$ (that is $\operatorname{ad}_{K, M}$ is injective), then $U(L)=J \oplus K$ (as vector spaces). 
Proof. (1) By Proposition 5.1.1 (4), it suffices to show that $\operatorname{dim} \widetilde{K}=\operatorname{dim} \frac{L}{C_{L}(M)}$. This will guarantee that the homomorphism $\tilde{\chi}$ in Proposition 5.1.1 (4) is an isomorphism. Since $L^{\prime} \subseteq M$ the Lie algebras $K=L / M$ and $\widetilde{K}$ are abelian. As $L / C_{L}(M)$ is a subalgebra of $\widetilde{K}$ it follows that $\operatorname{dim} \widetilde{K} \geqslant \operatorname{dim} \frac{L}{C_{L}(M)} \geqslant\left\lfloor\frac{(\operatorname{dim} M)^{2}}{4}\right\rfloor+1$. On the other hand, since $\operatorname{dim} \widetilde{I}=\operatorname{dim} M$, the maximal dimension of an abelian subalgebra of $\mathfrak{g l}(\widetilde{I})$ is $\left\lfloor\frac{(\operatorname{dim} M)^{2}}{4}\right\rfloor+1$, in virtue of $[12$, Theorem 1]. Thus, since $\widetilde{K}$ is a subalgebra of $\mathfrak{g l}(\widetilde{I})$, $\left\lfloor\frac{(\operatorname{dim} M)^{2}}{4}\right\rfloor+1 \geqslant \operatorname{dim} \tilde{K}$, and so $\operatorname{dim} \widetilde{K}=\operatorname{dim} \frac{L}{C_{L}(M)}$ must hold.

(2) First note that $J \cap K=J \cap L \cap K=C_{L}(M) \cap K=M \cap K=0$, see Proposition 5.1.1 (3). Now we will show that $U(L)=J+K$, this will imply that $U(L)=J \oplus K$. In item (1) we saw that $\operatorname{dim} K=\operatorname{dim} \widetilde{K}$; thus, if $\left\{x_{1}, \ldots, x_{d}\right\}$ is a basis of $K$ then $\left\{x_{1}+J, \ldots, x_{d}+J\right\}$ is a basis of $\widetilde{K}$. Hence, given $w \in U(L)$ we have that $w+J=\alpha_{1}\left(x_{1}+J\right)+\cdots+\alpha_{d}\left(x_{d}+J\right)$, and this implies that $w=\alpha_{1} x_{1}+\cdots+\alpha_{d} x_{d}+w_{1}$, where $w_{1} \in J$. Therefore, $U(L)=J+K$.

Corollary 5.1.3. Let $L$ be a finite-dimensional Lie algebra. Suppose that $M$ is an abelian ideal of $L$ and $x \in L$ such that $\left.(\operatorname{ad} x)^{2}\right|_{M}=\left.\operatorname{ad} x\right|_{M}$ and $L=C_{L}(M) \rtimes\langle x\rangle$. Choosing $\mathfrak{m}=\omega(L)$ we have that $L_{M} \cong M \rtimes \frac{L}{C_{L}(M)}$.

Proof. It is enough to verify that the homomorphism $\tilde{\chi}$ in Proposition 5.1.1 (4) is an isomorphism, this is equivalent to show that $\operatorname{dim} \widetilde{K}=1$. By Proposition 5.1.1 (3) $x \notin J$. We will show that $x^{2}-x \in J$, for this it is enough show that $\left[x^{2}-x, y\right] \in M \mathfrak{m}$ for all $y \in M$. Let us compute

$$
\begin{aligned}
{\left[x^{2}-x, y\right] } & =\left[x^{2}, y\right]-[x, y] \\
& =x[x, y]+[x, y] x-[x, y] \\
& \equiv x[x, y]-[x, y] \bmod M \mathfrak{m} \\
& \equiv[x,[x, y]]-[x, y] \bmod M \mathfrak{m}
\end{aligned}
$$

for all $y \in M$. Since $\left(\left.\operatorname{ad} x\right|_{M}\right)^{2}=\left.\operatorname{ad} x\right|_{M}$ it follows that $[x,[x, y]]-[x, y]=0$ for all $y \in M$, and this implies $\left[x^{2}-x, y\right] \in M \mathfrak{m}$. Hence $x^{2}-x \in J$. By Proposition 5.1.1 (2) $J \cap \omega(L)$ is an associative two-sided ideal of $U(L)$, this implies that $x^{3}-x^{2} \in J$. Thus $x^{3}-x=\left(x^{3}-x^{2}\right)+\left(x^{2}-x\right) \in J$. An inductive procedure implies that $x^{n}-x \in J$ for all $n \geqslant 1$, that is $x^{n} \equiv x \bmod J$ for all $n \geqslant 1$. Now, given an element $w \in U(L)$ it can be written as $w=\widetilde{w}+p(x)$, where $p(x)$ is a polynomial in $x$ and $\widetilde{w} \in C_{L}(M) U(L)$. 
By Proposition 5.1.1 (3) $\widetilde{w} \in J$ and since $x^{n} \equiv x$ for all $n \geqslant 1$ we have that $w \equiv \lambda x$ for some $\lambda \in \mathbb{F}$, and so $\widetilde{K}=U(L) / J=\langle x+J\rangle$. Thus $\operatorname{dim} \tilde{K}=1$ as we required.

Theorem 5.1.4. Let $L$ and $H$ be finite-dimensional Lie algebras over an arbitrary field $\mathbb{F}$ and let $M$ and $N$ be abelian ideals of $L$ and $H$, respectively. Suppose that $\alpha: U(L) \rightarrow U(H)$ is an algebra isomorphism such that $\alpha(M U(L))=N U(H)$. Let $\mathfrak{m}$ be a maximal ideal of $U(L)$ such that $U(L) / \mathfrak{m} \cong \mathbb{F}$ and $M \subseteq \mathfrak{m}$ and set $\mathfrak{n}=\alpha(\mathfrak{m})$. Then $\alpha$ induces an isomorphism between the Lie algebras $L_{M}$ and $H_{N}$.

Proof. Denote by $\widetilde{I}_{1}=M U(L) / M \mathfrak{m}$ and $\widetilde{I}_{2}=N U(H) / N \mathfrak{n}$. Since $\alpha(M U(L))=$ $N U(H)$ we have that

$$
\alpha(M \mathfrak{m})=\alpha(M U(L) \mathfrak{m})=\alpha(M U(L)) \alpha(\mathfrak{m})=N U(H) \mathfrak{n}=N \mathfrak{n}
$$

Thus, the map $\bar{\alpha}(a+M \mathfrak{m})=\alpha(a)+N \mathfrak{n}, a \in M U(L)$, is an isomorphism between $\widetilde{I}_{1}$ and $\widetilde{I}_{2}$.

Let $J_{1} / M U(L)$ and $J_{2} / N U(H)$ be the kernels of the actions of $U(L) / M U(L)$ and $U(H) / N U(H)$ on $\widetilde{I}_{1}$ and $\widetilde{I}_{2}$ defined by equation (5.2). We will show that $\alpha\left(J_{1}\right)=J_{2}$. By definition

$$
J_{1}=\{w \in U(L) \mid[w, a] \in M \mathfrak{m} \text { for all } a \in M U(L)\}
$$

Suppose that $w \in J_{1}$. Then $[w, a] \in M \mathfrak{m}$ for all $a \in M U(L)$. Now, since $\alpha(M \mathfrak{m})=N \mathfrak{n}$, it follows that $[\alpha(w), \alpha(a)]=\alpha([w, a]) \in N \mathfrak{n}$ for all $a \in M U(L)$. As $a$ runs through all elements of $M U(L), \alpha(a)$ runs through all elements of $N U(H)$, and so we have that $[\alpha(w), a] \in N \mathfrak{n}$ for all $a \in N U(H)$. Therefore $\alpha(w) \in J_{2}$, and so $\alpha\left(J_{1}\right) \subseteq J_{2}$ and the other inclusion can be proved similarly. Thus, the map $\widetilde{\alpha}\left(w+J_{1}\right)=\alpha(w)+J_{2}$, $w \in U(L)$, is an isomorphism between $U(L) / J_{1}$ and $U(H) / J_{2}$.

Define $(\bar{\alpha}, \widetilde{\alpha}): L_{M} \rightarrow H_{N}$, by $(\bar{\alpha}, \widetilde{\alpha})\left(a+M \mathfrak{m}, w+J_{1}\right)=\left(\bar{\alpha}(a+M \mathfrak{m}), \widetilde{\alpha}\left(w+J_{1}\right)\right)$. We show that $(\bar{\alpha}, \widetilde{\alpha})$ is an isomorphism, for this we require to show that

$$
\tilde{\alpha}\left(\left[a+M \mathfrak{m}, w+J_{1}\right]\right)=\left[\alpha(a)+N \mathfrak{n}, \alpha(w)+J_{2}\right]
$$

for all $a \in M U(L)$ and $w \in U(L)$. Let us compute

$$
\begin{aligned}
{\left[\alpha(a)+N \mathfrak{n}, \alpha(w)+J_{2}\right]=[\alpha(a), \alpha(w)]+} & N \mathfrak{n}= \\
& \alpha([a, w]+M \mathfrak{m})=\tilde{\alpha}\left(\left[a+M \mathfrak{m}, w+J_{1}\right]\right) .
\end{aligned}
$$

Hence $(\bar{\alpha}, \widetilde{\alpha})$ is an isomorphism, as claimed. 
Corollary 5.1.5. Suppose that $L$ and $H$ are finite-dimensional metabelian Lie algebras over an arbitrary field and that $\alpha: U(L) \rightarrow U(H)$ is an algebra isomorphism. Let $\mathfrak{m}$ be a maximal ideal of $U(L)$ such that $U(L) / \mathfrak{m} \cong \mathbb{F}$ and $M \subseteq \mathfrak{m}$ and set $\mathfrak{n}=\alpha(\mathfrak{m})$. Then $L_{L^{\prime}} \cong H_{H^{\prime}}$.

Proof. By Lemma 2.3.2 $\alpha\left(L^{\prime} U(L)\right)=H^{\prime} U(H)$. Thus, Theorem 5.1.4 implies that $L_{L^{\prime}} \cong H_{H^{\prime}}$

The following proposition analyzes the isomorphism problem for the Lie algebras of Group 5.2 in Section 4.4. This is another partial result towards the proof of Theorem 3.2.1,

Proposition 5.1.6. Suppose that $L$ belongs to Group 5.2 in Section 4.4. Then, choosing $\mathfrak{m}=\omega(L)$, we have that $L \cong L_{L^{\prime}}$. Consequently, if $L$ and $H$ are in Group 5.2 and $U(L) \cong U(H)$, then $L \cong H$ over an arbitrary field.

Proof. Note that $L=L^{\prime} \rtimes K$ where $K=L / L^{\prime}$, and also $C_{L}\left(L^{\prime}\right)=L^{\prime}$. Thus $\operatorname{dim} K=$ $\left\lfloor\frac{\left(\operatorname{dim} L^{\prime}\right)^{2}}{4}\right\rfloor+1$. Thus $L$ satisfies the hypotheses of Corollary 5.1.2, and applying item (1) of this corollary we conclude that $L_{L^{\prime}} \cong L^{\prime} \rtimes K=L$. Now, suppose that $L$ and $H$ are in Group 5.2 and that $\alpha: U(L) \rightarrow U(H)$ is an algebra isomomorphism. By Lemma 2.3.4 we can assume that $\alpha(\omega(L))=\omega(H)$. Thus, Corollary 5.1.5 implies that $L \cong L_{L^{\prime}} \cong H_{H^{\prime}} \cong H$.

\subsection{Computing $L_{M}$ for some Lie algebras}

In this section, we calculate $L_{M}$ for several Lie algebras $L$. In all cases we assume that $\mathfrak{m}=\omega(L)$. We recall that the base field $\mathbb{F}$ has arbitrary characteristic unless otherwise mentioned. At the end of this section, we will see that these calculations provide more examples of Lie algebras with non-isomorphic universal enveloping algebras.

(1) Let

$$
M^{2}=\left\langle x_{1}, x_{2}, x_{3}, x_{4} \mid\left[x_{4}, x_{1}\right]=x_{1},\left[x_{4}, x_{2}\right]=x_{2},\left[x_{4}, x_{3}\right]=x_{3}\right\rangle,
$$

see Group 4 in Section 3.1. To simplify the notation we set $L=M^{2}$. We will show that $L_{M} \cong L$, where $M=L^{\prime}$. In fact, note that $M=C_{L}(M)=\left\langle x_{1}, x_{2}, x_{3}\right\rangle$ and 
$L=M \rtimes\left\langle x_{4}\right\rangle$. It is immediate to verify that $\left.\left(\operatorname{ad} x_{4}\right)^{2}\right|_{M}=\left.\operatorname{ad} x_{4}\right|_{M}$. Thus, Corollary 5.1.3 implies that $L_{M} \cong M \rtimes\left\langle x_{4}\right\rangle=L$.

(2) Let $L$ be a nilpotent Lie algebra of class 2 , this is equivalent to $C_{L}\left(L^{\prime}\right)=L$. If $M=L^{\prime}$ then we have that $L_{M} \cong M$. In fact, by Proposition 5.1.1 (3) it follows that $\omega(L)=L U(L)=C_{L}(M) U(L) \subseteq J$. Therefore $J=U(L)$, and consequently $\widetilde{K}=0$. Hence $L_{M}=\widetilde{I} \cong M$. Examples of such Lie algebras include the $(2 n+1)$-dimensional Heisenberg Lie algebras, see Example 2.1.5 (4).

(3) Consider the Lie algebra

$$
M^{4}=\left\langle x_{1}, x_{2}, x_{3}, x_{4} \mid\left[x_{4}, x_{2}\right]=x_{3},\left[x_{4}, x_{3}\right]=x_{3}\right\rangle,
$$

see Group 3 in Section 3.1. To simplify the notation we set $L=M^{4}$. We calculate $L_{M}$, where $M=L^{\prime}$. We have that $M=\left\langle x_{3}\right\rangle$ and $C_{L}(M)=\left\langle x_{1}, x_{2}, x_{3}\right\rangle$. Thus $L / C_{L}(M)=$ $\left\langle x_{4}\right\rangle$ and $1=\operatorname{dim} \frac{L}{C_{L}(M)}=\left\lfloor\frac{(\operatorname{dim} M)^{2}}{4}\right\rfloor+1$. Hence, applying Corollary 5.1.2 (1), we obtain $L_{M} \cong\left\langle x_{3}\right\rangle \rtimes\left\langle x_{4}\right\rangle$, with the nonzero bracket $\left[x_{4}, x_{3}\right]=x_{3}$. Another way to obtain $L_{M}$ is to apply Corollary 5.1.3.

Now, let $N=\left\langle x_{1}, x_{2}, x_{3}\right\rangle$. We have that $C_{L}(N)=N$ and $L=C_{L}(N) \rtimes\left\langle x_{4}\right\rangle$. By observation it is immediate to verify that $\left.\left(\operatorname{ad} x_{4}\right)^{2}\right|_{N}=\left.\operatorname{ad} x_{4}\right|_{N}$, and therefore the hypotheses of Corollary 5.1 .3 are valid. Thus $L_{N} \cong N \rtimes\left\langle x_{4}\right\rangle \cong L$.

(4) Consider the Lie algebra

$$
M_{0}^{3}=\left\langle x_{1}, x_{2}, x_{3}, x_{4} \mid\left[x_{4}, x_{1}\right]=x_{1},\left[x_{4}, x_{2}\right]=x_{3},\left[x_{4}, x_{3}\right]=x_{3}\right\rangle,
$$

see Group 5 in Section 3.1. To simplify the notation we set $L=M_{0}^{3}$. We compute $L_{M}$, where $M=L^{\prime}$. We have that $M=\left\langle x_{1}, x_{3}\right\rangle$ and $C_{L}(M)=\left\langle x_{1}, x_{2}, x_{3}\right\rangle$. By observation we can verify the hypotheses of Corollary 5.1.3. Therefore

$$
L_{M} \cong M \rtimes \frac{L}{C_{L}(M)}=\left\langle x_{1}, x_{3}\right\rangle \rtimes\left\langle x_{4}\right\rangle,
$$

where $x_{4}$ acts as in $M_{0}^{3}$, that is via the transformation

$$
\left.\operatorname{ad} x_{4}\right|_{M}=\left(\begin{array}{ll}
1 & 0 \\
0 & 1
\end{array}\right) \text {. }
$$


Note that $L_{M} \cong L_{2}$, where $L_{2}$ is as Theorem 3.1.1.

If we choose $N=\left\langle x_{1}, x_{2}, x_{3}\right\rangle$ we have that $C_{L}(N)=N$. By observation it is immediate verify the hypotheses of Corollary 5.1 .3 with the ideal $N$. Thus $L_{N} \cong$ $N \rtimes \frac{L}{C_{L}(N)} \cong L$.

(5) Consider the Lie algebra

$$
M_{0, b}^{6}=\left\langle x_{1}, x_{2}, x_{3}, x_{4} \mid\left[x_{4}, x_{1}\right]=x_{2},\left[x_{4}, x_{2}\right]=x_{3},\left[x_{4}, x_{3}\right]=b x_{2}+x_{3}\right\rangle,
$$

see Group 5 in Section 3.1. As in (4), we set $L=M_{0, b}^{6}$. We calculate $L_{M}$, where $M=$ $L^{\prime}$. We have that $M=\left\langle x_{2}, x_{3}\right\rangle$ and $C_{L}(M)=\left\langle x_{1}, x_{2}, x_{3}\right\rangle$. By Proposition 5.1.1 (3), $x_{4} \notin J$. Let us calculate the action of $x_{4}$ and $x_{4}^{2}$ over $\widetilde{I}$

$$
\begin{aligned}
{\left[x_{4}, x_{2}\right] } & \equiv x_{3} \quad \bmod M \omega(L), \\
{\left[x_{4}, x_{3}\right] } & \equiv b x_{2}+x_{3} \quad \bmod M \omega(L), \\
{\left[x_{4}^{2}, x_{2}\right] } & \equiv b x_{2}+x_{3} \quad \bmod M \omega(L), \\
{\left[x_{4}^{2}, x_{3}\right] } & \equiv b x_{2}+(1+b) x_{3} \quad \bmod M \omega(L) .
\end{aligned}
$$

Thus $\left[x_{4}^{2}-x_{4}, x_{2}\right] \equiv b x_{2} \bmod M \omega(L)$, and $\left[x_{4}^{2}-x_{4}, x_{3}\right] \equiv b x_{3} \bmod M \omega(L)$. Note that $x_{4}^{2}-x_{4} \in J$ if and only if $b \neq 0$. Thus, we divide the analysis in to two cases:

If $b \neq 0$, then the nonzero elements $x_{4}+J, x_{4}^{2}-x_{4}+J$ are linearly independent. In fact, the matrices of the transformations induced by $x_{4}$ and $x_{4}^{2}-x_{4}$ on $\tilde{I}$ are

$$
\operatorname{ad}\left(x_{4}+J\right)=\left(\begin{array}{ll}
0 & b \\
1 & 1
\end{array}\right) \quad \text { and } \quad \operatorname{ad}\left(x_{4}^{2}-x_{4}+J\right)=\left(\begin{array}{ll}
b & 0 \\
0 & b
\end{array}\right)
$$

an these are linearly independent. Therefore $\operatorname{dim} U(L) / J \geqslant 2$. On the other hand, by [12, Theorem 1], $\operatorname{dim} U(L) / J \leqslant 2$, and so $\operatorname{dim} U(L) / J=2$. Hence

$$
L_{M}=\tilde{I} \rtimes \tilde{K}=\left\langle x_{2}+M \omega(L), x_{3}+M \omega(L)\right\rangle \rtimes\left\langle x_{4}+J, x_{4}^{2}-x_{4}+J\right\rangle,
$$

where the transformations induced by $x_{4}+J$ and $x_{4}^{2}-x_{4}+J$ are represented by the matrices in (5.5). Thus $L_{M}$ is isomorphic to Lie algebra given by the non-zero brackets

$$
\left[x_{3}, x_{1}\right]=x_{2},\left[x_{3}, x_{2}\right]=b x_{1}+x_{2},\left[x_{4}, x_{1}\right]=x_{1},\left[x_{4}, x_{2}\right]=x_{2}
$$


If $b=0$. Then $L=C_{L}(M) \rtimes\left\langle x_{4}\right\rangle$ and we observe that $\left.\left(\operatorname{ad} x_{4}\right)^{2}\right|_{M}=\left.\operatorname{ad} x_{4}\right|_{M}$. By Corollary 5.1 .3 it follows that

$$
L_{M} \cong M \rtimes \frac{L}{C_{L}(M)}=\left\langle x_{2}, x_{3}\right\rangle \rtimes\left\langle x_{4}\right\rangle,
$$

where $x_{4}$ acts as in $L$, that is via the transformation

$$
\left.\operatorname{ad} x_{4}\right|_{M}=\left(\begin{array}{ll}
0 & 0 \\
1 & 1
\end{array}\right)
$$

Note that this algebra is isomorphic to $L_{0}^{3}$, where $L_{0}^{3}$ is as Theorem 3.1.1.

(6) Consider the Lie algebra

$$
M_{0, c}^{7}=\left\langle x_{1}, x_{2}, x_{3}, x_{4} \mid\left[x_{4}, x_{1}\right]=x_{2},\left[x_{4}, x_{2}\right]=x_{3},\left[x_{4}, x_{3}\right]=c x_{2}\right\rangle,
$$

see Group 5 in Section 3.1. As in the items above we set $L=M_{0, c}^{7}$. We calculate $L_{M}$ where $M=L^{\prime}$. By Proposition 5.1.1 (3) $x_{4} \notin J$. Let us calculate the action of $x_{4}$ and $x_{4}^{2}$ over $\tilde{I}$

$$
\begin{aligned}
{\left[x_{4}, x_{2}\right] } & \equiv x_{3} \quad \bmod M \omega(L), \\
{\left[x_{4}, x_{3}\right] } & \equiv c x_{2} \quad \bmod M \omega(L), \\
{\left[x_{4}^{2}, x_{2}\right] } & \equiv c x_{2} \quad \bmod M \omega(L), \\
{\left[x_{4}^{2}, x_{3}\right] } & \equiv c x_{2} \quad \bmod M \omega(L) .
\end{aligned}
$$

Note that $x_{4}^{2} \in J$ if and only if $c=0$. In the same way as in (5) we divide the analysis in two cases:

If $c \neq 0$. Then, the non-zero elements $x_{4}+J$ and $x_{4}^{2}+J$ are linearly independent. In fact, the matrices of the transformations induced by $x_{4}$ and $x_{4}^{2}$ on $\widetilde{I}$ are

$$
\operatorname{ad}\left(x_{4}+J\right)=\left(\begin{array}{ll}
0 & c \\
1 & 0
\end{array}\right) \quad \text { and } \quad \operatorname{ad}\left(x_{4}^{2}+J\right)=\left(\begin{array}{cc}
c & 0 \\
0 & c
\end{array}\right)
$$

and these are linearly independent. Therefore $\operatorname{dim} U(L) / J \geqslant 2$. On other hand, by $[12$, Theorem 1$], \operatorname{dim} U(L) / J \leqslant 2$. Hence $\operatorname{dim} U(L) / J=2$, and it follows that $\widetilde{K}=\left\langle x_{4}+J, x_{4}^{2}+J\right\rangle$. Therefore

$$
L_{M}=\tilde{I} \rtimes \tilde{K}=\left\langle x_{2}+M \omega(L), x_{3}+M \omega(L)\right\rangle \rtimes\left\langle x_{4}+J, x_{4}^{2}+J\right\rangle,
$$


where the transformations induced by $x_{4}+J$ and $x_{4}^{2}+J$ are represented by the matrices in (5.7). Thus $L_{M}$ is isomorphic to Lie algebra given by the non-zero brackets

$$
\left[x_{3}, x_{1}\right]=x_{2},\left[x_{3}, x_{2}\right]=c x_{1},\left[x_{4}, x_{1}\right]=x_{1},\left[x_{4}, x_{2}\right]=x_{2}
$$

If $c=0$. Then $x_{4}^{2} \in J$. By Proposition 5.1.1 (2) $J \cap \omega(L)$ is a two-sided ideal, and this implies that any ordered monomial in which the degree of $x_{4}$ is at least 2 is in $J$. If $w \in U(L)$, we can write $w=\widetilde{w}+q\left(x_{4}\right)$, where $\widetilde{w} \in C_{L}(M) U(L)$ and $q\left(x_{4}\right)$ is a polynomial in $x_{4}$. By Proposition 5.1.1 (3) $\widetilde{w} \in J$, and since $x_{4}^{n} \in J$ for $n \geqslant 2$ the polynomial $q\left(x_{4}\right)$ has degree at most 1 modulo $J$; that is $w+J \in\left\langle x_{4}+J\right\rangle$. Hence $\widetilde{K}=\left\langle x_{4}+J\right\rangle$, and we have that $\operatorname{dim} \widetilde{K}=1$. Consequently, the homomorphism $\tilde{\chi}: M \rtimes \frac{L}{C_{L}(M)} \rightarrow L_{M}=\widetilde{I} \rtimes \widetilde{K}$ in Proposition 5.1.1 (4) is an isomorphism. Therefore

$$
L_{M}=\left\langle x_{2}, x_{3}\right\rangle \rtimes\left\langle x_{4}\right\rangle
$$

where $x_{4}$ acts as in $L$, that is via the transformation

$$
\operatorname{ad} x_{4}=\left(\begin{array}{ll}
0 & 0 \\
1 & 0
\end{array}\right) .
$$

Note $L_{M}$ is isomorphic to the 3 -dimensional Heisenberg Lie algebra $L_{0}^{4}$, see Theorem 3.1.1.

(7) Let $A=\left\langle x_{0}, \ldots, x_{p}\right\rangle$ be an abelian Lie algebra of dimension $p+1$ over a field $\mathbb{F}$ of characteristics $p$. Consider the Lie algebra $L=A \rtimes\langle\lambda, \pi\rangle$ with the relations given by $\left[\lambda, x_{i}\right]=x_{i-1}$ for $1 \leqslant i \leqslant p$ and $\left[\lambda, x_{0}\right]=0,\left[\pi, x_{p}\right]=x_{0}$ and $\left[\pi, x_{i}\right]=0$ for $0 \leqslant i \leqslant p-1,[\lambda, \pi]=0$; see 27 , Example B]. We choose $M=L^{\prime}$, and we calculate the algebra $L_{M}$. We have that $M=\left\langle x_{0}, \ldots, x_{p-1}\right\rangle$ and $C_{L}(M)=\langle A, \pi\rangle$. By Proposition 4.4.4 (3) it follows that $\lambda \notin J$. The action of $\lambda+J$ on $\tilde{I}$, in the basis $\left\{x_{0}+M \omega(L), \ldots, x_{p-1}+M \omega(L)\right\}$, is given by

$$
\operatorname{ad}(\lambda+J)=\left(\begin{array}{c|c}
0 & I_{p-1} \\
\hline 0 & 0
\end{array}\right)
$$

where $I_{p-1}$ is the identity matrix of size $(p-1) \times(p-1)$, and the other blocks have the adequate size. 
We will show by induction, for $1 \leqslant k \leqslant p-1$, that

$$
\operatorname{ad}\left(\lambda^{k}+J\right)=\left(\begin{array}{c|c}
0 & I_{p-k} \\
\hline 0 & 0
\end{array}\right),
$$

where $I_{p-k}$ is the identity matrix of size $(p-k) \times(p-k)$, and the other blocks have the adequate size. By $(5.8)$ this is true for $k=1$. Note that the induction hypothesis is equivalent to

$$
\left[\lambda^{k}, x_{i}\right] \equiv\left\{\begin{array}{cl}
0 ; & \text { if } i<k \\
x_{i-k} ; & \text { if } i \geqslant k
\end{array} \quad \bmod M \omega(L) .\right.
$$

Since $\left[\lambda, x_{i}\right] \lambda^{k} \in M \omega(L)$, we obtain that

$$
\begin{aligned}
{\left[\lambda^{k+1}, x_{i}\right] } & =\lambda\left[\lambda^{k}, x_{i}\right]+\left[\lambda, x_{i}\right] \lambda^{k} \\
& \equiv\left\{\begin{array}{cl}
0 ; & \text { if } i<k \\
\lambda x_{i-k} ; & \text { if } i \geqslant k
\end{array} \quad \bmod M \omega(L)\right. \\
& \equiv\left\{\begin{array}{cl}
0 ; & \text { if } i<k+1 \\
x_{i-(k+1)} ; & \text { if } i \geqslant k+1
\end{array} \bmod M \omega(L) .\right.
\end{aligned}
$$

Note that we use the induction hypothesis in the second line. Thus, equation (5.9) holds. Hence, we can conclude, by the definition of $J$ (see equation $(5.3)$ ), that $\lambda^{k} \notin J$ for $1 \leqslant k \leqslant p-1$.

Since char $\mathbb{F}=p$ equation $(2.1)$ implies that

$$
\operatorname{ad} \lambda^{p}\left(x_{i}\right)=(\operatorname{ad} \lambda)^{p}\left(x_{i}\right)=0,
$$

for $0 \leqslant i \leqslant p-1$. Thus, $\lambda^{p} \in J$. By Proposition 5.1.1 (2) $J \cap \omega(L)$ is a two sided ideal. Thus any ordered monomial in which the degree of $\lambda$ is at least $p$ is in $J$. Now, if $w \in U(L)$, then we can write $w=\widetilde{w}+q(\lambda)$, where $\widetilde{w} \in C_{L}(M) U(L)$ and $q(\lambda)$ is a polynomial in $\lambda$. Proposition 5.1.1 (3) implies that $\widetilde{w} \in J$, and since $\lambda^{p} \in J$ we have that the polynomial $q(\lambda)$ has degree at most $p-1$ modulo $J$. That is $w+J \in\left\langle\lambda+J, \ldots, \lambda^{p-1}+J\right\rangle$. In short, we have shown that the set $\left\{\lambda+J, \ldots, \lambda^{p-1}+J\right\}$ span $\tilde{K}=U(L) / J$. Since the matrices in (5.8) and (5.9) are linearly independent, then so is the set $\left\{\lambda+J, \ldots, \lambda^{p-1}+J\right\}$. Therefore, we have that

$$
\tilde{K}=U(L) / J=\left\langle\lambda+J, \ldots, \lambda^{p-1}+J\right\rangle .
$$


Finally, $L_{M}=\widetilde{I} \rtimes \widetilde{K}$ is the $(2 p-1)$-dimensional Lie algebra with the action of $\widetilde{K}$ on $\tilde{I}$ given by the matrices in (5.8) and $(5.9)$.

With the calculations made so far we can show the following proposition which is a partial result towards the proof of Theorem 3.2.1.

Proposition 5.2.1. In Group 5.1 (see final of Section 4.4) for an arbitrary field $\mathbb{F}$ we have that

$$
\begin{aligned}
& \text { 1. } U\left(M_{0}^{3}\right) \not U\left(M_{0, b}^{6}\right) \text {; } \\
& \text { 2. } U\left(M_{0}^{3}\right) \not U\left(M_{0, c}^{7}\right) \text {; } \\
& \text { 3. } U\left(M_{0,0}^{6}\right) \not U\left(M_{0,0}^{7}\right) \text {. }
\end{aligned}
$$

Proof. (1) If $U\left(M_{0}^{3}\right) \cong U\left(M_{0, b}^{6}\right)$, then Corollary 5.1 .5 implies that the Lie algebras obtained in items (4) and (5) are isomorphic, which is a contradiction.

(2) If $U\left(M_{0}^{3}\right) \cong U\left(M_{0, c}^{7}\right)$, then Corollary 5.1.5 implies that the Lie algebras obtained in items (4) and (6) are isomorphic, which is a contradiction.

(3) If $U\left(M_{0,0}^{6}\right) \cong U\left(M_{0,0}^{7}\right)$, then Corollary 5.1 .5 implies that $L_{0}^{3} \cong L_{0}^{4}$, where $L_{0}^{3}$ and $L_{0}^{4}$ were obtained in items (5) and (6), respectively. But, Theorem 3.1.1 says that $L_{0}^{3} \nRightarrow L_{0}^{4}$. Hence $U\left(M_{0, b}^{6}\right) \nRightarrow U\left(M_{0, c}^{7}\right)$. 


\section{Chapter 6}

\section{Solvable Lie algebras with an ideal of codimension one}

In this chapter, we study the structure of the universal enveloping algebra $U(L)$ for Lie algebras of the form $L=M \rtimes\langle x\rangle$, where $M$ is an ideal of $L$. These Lie algebras have some advantages. First, the complement (as vector spaces) of $M$ is a Lie algebra; in general this is not true if $\operatorname{dim}(L / M) \geqslant 2$. Second, $U(L / M)$ can be viewed as the polynomial ring $\mathbb{F}[x]$; and the automorphisms of $\mathbb{F}[x]$ are well understood. We will exploit this.

This chapter consists of two sections. In Section 6.1, we investigate the structure of the universal enveloping algebras for Lie algebras of the form $L=M \rtimes\langle x\rangle$. The main result of this section is Proposition 6.1.1, which basically says that under some conditions the isomorphism type of $L / M^{\prime}$ is determined by the isomorphism type of $U(L)$. As an application of the results in Section 6.1 we show that the isomorphism problem has positive solution for 3-dimensional solvable Lie algebras over an arbitrary field. Originally this theorem was shown by Chun, Kajiwara and Lee in [4] and the proof presented here is in essence the same as in [4]. Finally, in Section 6.2 we present the final part of the proof of Theorem 3.2.1, which is the main result of this thesis.

\subsection{Lie algebras with ideals of codimension 1}

The following result was inspired by a corresponding result for group rings originally proved by Whitcomb in [33], see also [24, Theorem 9.3.13]. With this result 
Whitcomb showed that the isomorphism problem for integral group rings has a positive solution for metabelian groups. In the proof we use a well know fact that if $\varphi$ is an automorphism of a polynomial ring $\mathbb{F}[x]$, then $\varphi(x)=a x+b$ with $a, b \in \mathbb{F}$ and $a \neq 0$; see 20 , Proposition 3.1].

Proposition 6.1.1. Let $L=M \rtimes\langle x\rangle$ and $H=N \rtimes\langle y\rangle$ be finite-dimensional Lie algebras over an arbitrary field, where $M$ and $N$ are ideals of $L$ and $H$, respectively. Suppose that $\alpha: U(L) \rightarrow U(H)$ is an algebra isomorphism such that $\alpha(M U(L))=$ $N U(H)$. Then, $L / M^{\prime} \cong H / N^{\prime}$.

Proof. Let $\bar{\alpha}: U(L) \rightarrow U(H)$ be the isomorphism constructed in Lemma 2.3.4. Since $\alpha(m) \in \omega(H)$ holds for all $m \in M$, we obtain that $\bar{\alpha}(M)=\alpha(M)$. Therefore

$$
\bar{\alpha}(M U(L))=\bar{\alpha}(M) \bar{\alpha}(U(L))=\alpha(M) \alpha(U(L))=\alpha(M U(L))=N U(H) .
$$

Thus, we may assume without loss of generality that $\alpha$ satisfies $\alpha(\omega(L))=\omega(H)$ and in addition $\alpha(M U(L))=N U(H)$.

We claim that

$$
\text { for all } z \in L \text { there exists } y_{z} \in H \text { such that } \alpha(z) \equiv y_{z} \bmod N \omega(H) \text {. }
$$

Let us first verify claim (6.1) in the case when $z=x$. The map $\alpha$ induces an isomorphism between $U(L) / M U(L)$ and $U(H) / N U(H)$. On the other hand, by Lemma 2.3.1 (2),

$$
U(L) / M U(L) \cong U(L / M) \quad \text { and } \quad U(H) / N U(H) \cong U(H / N)
$$

and so we can view $U(L) / M U(L)$ and $U(H) / N U(H)$ as polynomial rings in the variables $x+M U(L)$ and $y+N U(H)$, respectively. Thus, using the remark preceding the proposition, we have $\alpha(x)+N U(H)=a y+b+N U(H)$ where $a, b \in \mathbb{F}$ and $a \neq 0$, and as we assume $\alpha(\omega(L))=\omega(H)$ it follows that $b=0$. Hence, $\alpha(x)+N U(H)=$ $a y+N U(H)$, and it follows that there exist $z_{1}, \ldots, z_{n} \in N$ and $u_{1}, \ldots, u_{n} \in U(H)$ such that

$$
\alpha(x)=a y+\sum_{i=1}^{n} z_{i} u_{i} .
$$


Letting $\varepsilon_{H}$ denote the augmentation map of $U(H)$, we obtain

$$
\begin{aligned}
\alpha(x) & =a y+\sum_{i=1}^{n} z_{i} u_{i}=a y+\sum_{i=1}^{n} z_{i}\left(u_{i}-\varepsilon_{H}\left(u_{i}\right)+\varepsilon_{H}\left(u_{i}\right)\right) \\
& =a y+\sum_{i=1}^{n} \varepsilon_{H}\left(u_{i}\right) z_{i}+\sum_{i=1}^{n} z_{i}\left(u_{i}-\varepsilon_{H}\left(u_{i}\right)\right) .
\end{aligned}
$$

Since $u_{i}-\varepsilon_{H}\left(u_{i}\right) \in \omega(H)$, it follows that

$$
\alpha(x) \equiv a y+\sum_{i=1}^{n} \varepsilon_{H}\left(u_{i}\right) z_{i} \bmod N \omega(H) .
$$

Note that the element on the right-hand side of equation 6.2 lies in $H$. Denoting this element by $y_{x}$, we obtain that

$$
\alpha(x) \equiv y_{x} \quad \bmod N \omega(H)
$$

Hence property (6.1) is valid for the element $x \in L$. If $z \in M$; then, by hypothesis, $\alpha(z) \in N U(H)=N+N \omega(H)$, and so there is some $y_{z} \in N$ such that $\alpha(z) \equiv y_{z}$ $\bmod N \omega(H)$. Therefore property (6.1) holds also for elements of $M$. Since $L=$ $M+\langle x\rangle$, claim (6.1) must hold for all elements $z \in L$.

Now, using Lemma 2.3.1 (3) we have that

$$
\alpha(M \omega(L))=\alpha(M U(L) \omega(L))=\alpha(M U(L)) \alpha(\omega(L))=N U(H) \omega(H)=N \omega(H) .
$$

Therefore the assignment $z+M \omega(L) \mapsto y_{z}+N \omega(H)$, for all $z \in L$, defines an Lie homomorpism between $(L+M \omega(L)) / M \omega(L)$ and $(H+N \omega(H)) / N \omega(H)$. We show that this homomorphism is injective. A coset $z+M \omega(L)$ is in the kernel of this map if and only if $y_{z} \in N \omega(H)$; this implies, by definition of $y_{x}$, that $\alpha(z) \in N \omega(H)$. Applying $\alpha^{-1}$ in the last equality we have that $z \in M \omega(L)$. Therefore, $z+M \omega(L)=$ $M \omega(L)$, and it follows that the kernel of this map is trivial. Hence, the map in the middle of the following expression is injective

$$
\frac{L}{L \cap M \omega(L)} \cong \frac{L+M \omega(L)}{M \omega(L)} \rightarrow \frac{H+N \omega(H)}{N \omega(H)} \cong \frac{H}{H \cap N \omega(H)} .
$$

Applying Lemma 2.3.1 (4), we obtain that $L /(L \cap M \omega(L)) \cong L / M^{\prime}$ and, similarly, that $H /(H \cap N \omega(H)) \cong H / N^{\prime}$. Thus there exists an injective Lie algebra homomorphism 
$L / M^{\prime} \rightarrow H / N^{\prime}$. On the other hand, Lemma 2.3 .3 (6) shows that $\operatorname{dim} M / M^{\prime}=$ $\operatorname{dim} N / N^{\prime}$, and since $\operatorname{dim} L / M=\operatorname{dim} H / N=1$ we can conclude that $\operatorname{dim} L / M^{\prime}=$ $\operatorname{dim} H / N^{\prime}$. Now it follows that the injective homomorphism $L / M^{\prime} \rightarrow H / N^{\prime}$ is an isomorphism.

Corollary 6.1.2. Suppose that $L$ and $H$ are Lie algebras as Proposition 6.1.1 and that $\alpha: U(L) \rightarrow U(H)$ is an algebra isomorphism. Then the following are valid.

1. If $\alpha(M U(L))=N U(H)$ and either $M$ or $N$ is abelian, then $L \cong H$.

2. If $M=L^{\prime}$ and $N=H^{\prime}$, then $L / L^{\prime \prime} \cong H / H^{\prime \prime}$. In particular; if either $L$ or $H$ is metabelian, then $L \cong H$.

Proof. (1) First we note that by Lemma 2.3.3 (1) $\operatorname{dim} L=\operatorname{dim} H$. Suppose without loss of generality that $M$ is abelian. Since $\alpha(M U(L))=N U(H)$, Proposition 6.1.1 shows that $L \cong H / N^{\prime}$, and hence $N^{\prime}=0$. Thus $L \cong H$, as claimed.

(2) Lemma 2.3.2 implies that $\alpha\left(L^{\prime} U(L)\right)=H^{\prime} U(H)$. Thus, by Proposition 6.1.1. with $M=L^{\prime}$ and $N=H^{\prime}$, gives us $L / L^{\prime \prime} \cong H / H^{\prime \prime}$.

The following corollary states that the isomorphism problem has a positive solution in the class of solvable Lie algebras of dimension 3 over an arbitrary field (we promised a proof of this fact in Section 3.2). Originally this was shown by Chun, Kajiwara and Lee in [4]; in this paper they showed that the isomorphism problem has a positive solution in the class of 3-dimensional Lie algebra over a field of characteristic different from two. But we observe that in the solvable case this affirmation is independent of the characteristic of the field.

Corollary 6.1.3 ([4]). Let $L, H$ be solvable Lie algebras of dimension 3 over an arbitrary field. If $U(L) \cong U(H)$, then $L \cong H$.

Proof. The proof of this corollary basically follows the same steps as Theorem 3.2.1. By Theorem 3.1.1 there exist 4 families of isomorphism classes of solvable Lie algebras of dimension 3. We divide these families into three groups in a way that two Lie algebras in different groups cannot have isomorphic universal enveloping algebras. Later, we analyze each group in order to ensure that in the same group do not exist two non-isomorphic Lie algebras with isomorphic universal enveloping algebras. The 
proof of Theorem 6.1 .3 follows from these two steps. For the group division we use the same notation as in Theorem 3.1.1 and we refer to this theorem for all information about these algebras.

Group 1 (Abelian Lie algebra): $L^{1}$.

Group 2 (Lie algebras with two-dimensional derived algebra): $L^{2}, L_{a}^{3}$ with $a \in \mathbb{F}^{\times}$and $L_{a}^{4}$ with $a \in \mathbb{F}^{\times}$.

Group 3 (Lie algebras with one-dimensional derived algebra): $L_{0}^{3}$ and $L_{0}^{4}$.

Claim 1: If $L$ an $H$ are Lie algebras in different Groups, then $U(L) \nsubseteq U(H)$ over an arbitrary field $\mathbb{F}$.

Proof: Item (1) Lemma 2.3.3 imply that the isomorphism type of $U(L)$ determines whether $L$ is abelian and determines $\operatorname{dim} L^{\prime}$. Thus, Claim 1 follows.

Claim 2: If $L$ and $H$ are Lie algebras in Group 2 over an arbitrary field $\mathbb{F}$; such that $U(L) \cong U(H)$ then $L \cong H$.

Proof: Note that $L$ and $H$ are metabelian and that $L=L^{\prime} \rtimes\left\langle x_{3}\right\rangle$ and $H=H^{\prime} \rtimes\left\langle x_{3}\right\rangle$. Hence if $U(L) \cong U(H)$, then Corollary 6.1.2 (2) implies that $L \cong H$.

Claim 3: In Group 3, we have that $U\left(L_{0}^{3}\right) \not U\left(L_{0}^{4}\right)$ over an arbitrary field $\mathbb{F}$.

Proof: This follows at once from Lemma 2.3.3 (2), as $L_{0}^{3}$ is non-nilpotent, but $L_{0}^{4}$ is nilpotent.

The proof of Corollary 6.1 .3 follows from the three claims.

As another application of Corollary 6.1.2 in the next section we analyze the isomorphism problem for Group 2 and Group 4, see Section 3.1.

Let $L$ be a finite-dimensional non-abelian metabelian Lie algebra. Suppose that $Z(L) \neq 0$. Then it is clear that $M=L^{\prime}+Z(L)$ is an abelian ideal of $L$ and $M \subseteq$ $C_{L}\left(L^{\prime}\right)$. Let us assume that $M$ has codimension 1 on $L$, that is $L=M \rtimes\langle x\rangle$, for some $x \in L$. Since $M \subseteq C_{L}\left(L^{\prime}\right)$ we have two possibilities: $M=C_{L}\left(L^{\prime}\right)$ or $L=C_{L}\left(L^{\prime}\right)$. If we assume that $L=C_{L}\left(L^{\prime}\right)$, then $L$ is abelian which is a contradiction. Thus $M=C_{L}\left(L^{\prime}\right)$, and there exists $y \in L^{\prime}$ such that $[y, x] \neq 0$, and it follows that $C_{L}(y)$ is a proper subspace of $L$. On the other hand, it is clear that $M \subseteq C_{L}(y)$, and this implies that $M=C_{L}(y)$. Furthermore, if we assume that char $\mathbb{F}=0$, then we can 
apply items (2) and (1) of Lemma 4.4.4 and conclude that $F(L) \subseteq M$, where $F(L)$ denotes the Frobenius semiradical of the Lie algebra $L$, see Section 4.4 .

In the following proposition we show that if two finite-dimensional metabelian Lie algebras of the form $L=\left(L^{\prime}+Z(L)\right) \rtimes\langle x\rangle$ have isomorphic universal enveloping algebras over a field of characteristic 0 , then these Lie algebras must be isomorphic.

Proposition 6.1.4. Suposse that $L$ and $H$ are two finite-dimensional metabelian Lie algebras over a field $\mathbb{F}$ of characteristic 0 , and that $L=\left(L^{\prime}+Z(L)\right) \rtimes\langle x\rangle$ and $H=\left(H^{\prime}+Z(H)\right) \rtimes\langle y\rangle$. If $\alpha: U(L) \rightarrow U(H)$ is an algebra isomorphism, then $L \cong H$.

Proof. First, we note that the result is true if either one $L$ or $H$ is abelian. Therefore, we assume that $L$ and $H$ are non-abelian. Now, let us denote $M=L^{\prime}+Z(L)$ and $N=H^{\prime}+Z(H)$, by the discussion above it follows that $F(L) \subseteq M$ and $F(H) \subseteq N$. Applying Lemma 4.4.4 (3), we have the following chains of inclusions:

$$
Z(U(L)) \subseteq U(F(L)) \subseteq U(M) \text { and } \quad Z(U(H)) \subseteq U(F(H)) \subseteq U(N)
$$

We may assume, using Lemma 2.3.4, that $\alpha(\omega(L))=\omega(H)$. We will show that $\alpha(M U(L))=N U(H)$, and since $M$ and $N$ are abelian ideals the proof follows directly from Corollary 6.1.2 (1).

Lemma 2.3.2 implies that $L^{\prime} U(L)$ is invariant by $\operatorname{Aut}(U(L))$. Hence

$$
\alpha\left(L^{\prime} U(L)\right)=H^{\prime} U(H) \subseteq N U(H),
$$

and since $Z(L) \subseteq Z(U(L))$ it follows that

$$
\alpha(Z(L)) \subseteq Z(U(H))
$$

Further, $Z(L) \subseteq \omega(L)$, and hence we obtain from equation (6.4) that

$$
\alpha(Z(L)) \subseteq Z(U(H)) \cap \omega(H) \subseteq U(N) \cap \omega(H)=\omega(N) \subseteq N U(H) .
$$

Thus $\alpha(Z(L) U(L)) \subseteq N U(H)$, and

$$
\alpha(M U(L))=\alpha\left(L^{\prime} U(L)\right)+\alpha(Z(L) U(L)) \subseteq N U(H) .
$$

Applying the same argument to the isomorphism $\alpha^{-1}: U(H) \rightarrow U(L)$ we have that $\alpha^{-1}(N U(H)) \subseteq M U(L)$. Therefore $\alpha(M U(L))=N U(H)$, and since $M$ and $N$ are abelian ideals of $L$ and $H$, respectively; Corollary 6.1.2 (1) implies that $L \cong H$. 
As an application of Proposition 6.1.4 we will show in the next section that the Lie algebras $M_{0, b}^{6}$ and $M_{0, c}^{7}$ has non-isomorphic universal enveloping algebras. With which we conclude the analysis of the isomorphism problem in Group 5.1 of Section 4.4.

\subsection{The final part of the proof of Theorem 3.2.1}

In this section, we present the final part of the proof of Theorem 3.2.1. The only thing left to do is to analyze the isomorphism problem in Groups 2 and 4 and finish the analysis in Group 5.1. After this, the only thing that remains is to put all the pieces together.

Proposition 6.2.1. Let $L$ and $H$ are Lie algebras in Group 4 such that $U(L) \cong U(H)$, then $L \cong H$ for an arbitrary field $\mathbb{F}$.

Proof. We have that $L=L^{\prime} \rtimes\left\langle x_{4}\right\rangle$ and $H=H^{\prime} \rtimes\left\langle x_{4}\right\rangle$ and that $L^{\prime}$ and $H^{\prime}$ are abelian. Hence if $U(L) \cong U(H)$, then Corollary 6.1.2 (2) implies that $L \cong H$.

Proposition 6.2.2. Let $L$ and $H$ Lie algebras in Group 2 such that $U(L) \cong U(H)$, then $L \cong H$ for a field $\mathbb{F}$ of characteristic 0 .

Proof. Suppose that $L$ and $H$ are two Lie algebras in Group 2, we have that $L=$ $L^{\prime} \rtimes\left\langle x_{4}\right\rangle$ and $H=H^{\prime} \rtimes\left\langle x_{4}\right\rangle$. Since the Lie algebra $M_{a, b}^{11}$ only exists in characteristic two, we omitted this from the analysis. We will show that if $L \not H$ then $L / L^{\prime \prime} \not H / H^{\prime \prime}$, and Corollary $6.1 .2(2)$ will imply $U(L) \nRightarrow U(H)$. It is easy to verify that

$$
\left(M^{12}\right)^{\prime \prime}=\left\langle x_{2}\right\rangle, \quad\left(M_{a}^{13}\right)^{\prime \prime}=\left\langle x_{2}\right\rangle, \quad\left(M_{a}^{14}\right)^{\prime \prime}=\left\langle x_{2}\right\rangle .
$$

Moreover

$$
\begin{aligned}
& M^{12} /\left(M^{12}\right)^{\prime \prime}=\left\langle\overline{x_{1}}, \overline{x_{3}}, \overline{x_{4}} \mid\left[\overline{x_{4}}, \overline{x_{1}}\right]=\overline{x_{1}},\left[\overline{x_{4}}, \overline{x_{3}}\right]=\overline{x_{3}}\right\rangle ; \\
& M_{a}^{13} /\left(M_{a}^{13}\right)^{\prime \prime}=\left\langle\overline{x_{1}}, \overline{x_{3}}, \overline{x_{4}} \mid\left[\overline{x_{4}}, \overline{x_{1}}\right]=\overline{x_{1}}+a \overline{x_{3}},\left[\overline{x_{4}}, \overline{x_{3}}\right]=\overline{x_{3}}\right\rangle ; \\
& M_{a}^{14} /\left(M_{a}^{14}\right)^{\prime \prime}=\left\langle\overline{x_{1}}, \overline{x_{3}}, \overline{x_{4}} \mid\left[\overline{x_{4}}, \overline{x_{1}}\right]=a \overline{x_{3}},\left[\overline{x_{4}}, \overline{x_{3}}\right]=\overline{x_{2}}\right\rangle .
\end{aligned}
$$


Where $\overline{x_{i}}$ denote the image of $x_{i}$ in the quotient, for $i=1,3,4$. Now, by simple observation we obtained the following isomorphisms

$$
\begin{aligned}
& M^{12} /\left(M^{12}\right)^{\prime \prime} \cong L^{2} \\
& M_{a}^{13} /\left(M_{a}^{13}\right)^{\prime \prime} \cong L_{a}^{3} \\
& M_{a}^{14} /\left(M_{a}^{14}\right)^{\prime \prime} \cong L_{a}^{4}
\end{aligned}
$$

see Theorem 3.1.1. Hence, if $L \not H$, then $L / L^{\prime \prime} \not H / H^{\prime \prime}$. Therefore, if $U(L) \cong U(H)$ then we must have $L \cong H$.

We will show the following proposition with which we conclude the analysis of the isomorphism problem in Group 5.1 of Section 4.4 .

Proposition 6.2.3. Suppose that $\mathbb{F}$ is a field of characteristic 0 and $b, c \in \mathbb{F}$ are non-zero. Then $U\left(M_{0, b}^{6}\right) \nRightarrow U\left(M_{0, c}^{7}\right)$. Consequently, if $L$ and $H$ are Lie algebras in Group 5.1 such that $U(L) \cong U(H)$ then $L \cong H$ over the field $\mathbb{F}$.

Proof. We have that $M_{0, b}^{6}$ and $M_{0, c}^{7}$ are metabelian and $Z\left(M_{0, b}^{6}\right)=\left\langle b x_{1}+x_{2}-x_{3}\right\rangle$ and $Z\left(M_{0, c}^{7}\right)=\left\langle c x_{1}-x_{3}\right\rangle$, see Section 3.1. Therefore, it follows that $M_{0, b}^{6}=\left(\left(M_{0, b}^{6}\right)^{\prime}+\right.$ $\left.Z\left(M_{0, b}^{6}\right)\right) \rtimes\left\langle x_{4}\right\rangle$ and $M_{0, c}^{7}=\left(\left(M_{0, c}^{7}\right)^{\prime}+Z\left(M_{0, c}^{7}\right)\right) \rtimes\left\langle x_{4}\right\rangle$. Since $M_{0, b}^{6} \not M_{0, c}^{7}$, Proposition 6.1 .4 implies that $U\left(M_{0, b}^{6}\right) \not U\left(M_{0, c}^{7}\right)$. Moreover, putting together this with Proposition 5.2.1 we have that if $L$ and $H$ are Lie algebras in Group 5.1 such that $U(L) \cong U(H)$, then $L \cong H$

At this point we have all the necessary pieces for the proof of Theorem 3.2.1. the only thing that remains is to put them together. As we said in Section 3.2 to prove Theorem 3.2.1 we only needed to complete step two. That is, to ensure that in each group, of the group division in Section 3.1, do not exists two Lie algebras with isomorphic universal enveloping algebras.

Proof of Theorem 3.2.1: Putting together Proposition 3.2.2, Lemma 3.2.3. Proposition 4.4.12, Proposition 5.1.6, Proposition 6.2.1, Proposition 6.2.2 and Proposition 6.2 .3 . 


\section{Bibliography}

[1] George M. Bergman, The diamond lemma for ring theory, Adv. in Math. 29 (1978), no. 2, 178-218. MR506890

[2] Nicolas Bourbaki, Lie groups and Lie algebras. Chapters 1-3, Elements of Mathematics (Berlin), Springer-Verlag, Berlin, 1998. Translated from the French, Reprint of the 1989 English translation. MR1728312

[3] Amiram Braun and Gil Vernik, On the center and semi-center of enveloping algebras in prime characteristic, J. Algebra 322 (2009), no. 5, 1830-1858. MR2543637

[4] Jang-Ho Chun, Takeshi Kajiwara, and Jong-Sook Lee, Isomorphism theorem on low dimensional Lie algebras, Pacific J. Math. 214 (2004), no. 1, 17-21. MR2039123

[5] W. A. de Graaf, Classification of solvable Lie algebras, Experiment. Math. 14 (2005), no. 1, 15-25. MR2146516

[6] Willem A. de Graaf, Classification of 6-dimensional nilpotent Lie algebras over fields of characteristic not 2, J. Algebra 309 (2007), no. 2, 640-653. MR2303198

[7] L. Delvaux, E. Nauwelaerts, and A. I. Ooms, On the semicenter of a universal enveloping algebra, J. Algebra 94 (1985), no. 2, 324-346. MR792958

[8] Jacques Dixmier, Algèbres enveloppantes, Les Grands Classiques Gauthier-Villars. [GauthierVillars Great Classics], Éditions Jacques Gabay, Paris, 1996. Reprint of the 1974 original. MR1451138

[9] Alexander G. Elashvili and Alfons I. Ooms, On commutative polarizations, J. Algebra 264 (2003), no. 1, 129-154. MR1980689

[10] G. Hochschild, An addition to Ado's theorem, Proc. Amer. Math. Soc. 17 (1966), 531-533. MR0194482

[11] James E. Humphreys, Introduction to Lie algebras and representation theory, Graduate Texts in Mathematics, vol. 9, Springer-Verlag, New York-Berlin, 1978. Second printing, revised. MR499562

[12] N. Jacobson, Schur's theorems on commutative matrices, Bull. Amer. Math. Soc. 50 (1944), 431-436. MR0010540

[13] Nathan Jacobson, Lie algebras, Dover Publications, Inc., New York, 1979. Republication of the 1962 original. MR559927

[14] T. Y. Lam, A first course in noncommutative rings, Second, Graduate Texts in Mathematics, vol. 131, Springer-Verlag, New York, 2001. MR1838439 
[15] P. Malcolmson, Enveloping algebras of simple three-dimensional Lie algebras, J. Algebra 146 (1992), no. 1, 210-218. MR1152441

[16] J. C. McConnell and J. C. Robson, Noncommutative Noetherian rings, Revised, Graduate Studies in Mathematics, vol. 30, American Mathematical Society, Providence, RI, 2001. With the cooperation of L. W. Small. MR1811901

[17] John W. Milnor and John C. Moore, On the structure of Hopf algebras, Ann. of Math. (2) 81 (1965), 211-264. MR0174052

[18] Susan Montgomery, X-inner automorphisms of filtered algebras, Proc. Amer. Math. Soc. 83 (1981), no. 2, 263-268. MR624911

[19] _ Hopf algebras and their actions on rings, CBMS Regional Conference Series in Mathematics, vol. 82, Published for the Conference Board of the Mathematical Sciences, Washington, DC; by the American Mathematical Society, Providence, RI, 1993. MR1243637

[20] Masayoshi Nagata, On automorphism group of $k[x, y]$, Kinokuniya Book-Store Co., Ltd., Tokyo, 1972. Department of Mathematics, Kyoto University, Lectures in Mathematics, No. 5. MR0337962

[21] Alfons I. Ooms, The Frobenius semiradical of a Lie algebra, J. Algebra 273 (2004), no. 1, 274287. MR2032460

[22] Inder Bir S. Passi and Sudarshan K. Sehgal, Isomorphism of modular group algebras, Math. Z. 129 (1972), 65-73. MR0311752

[23] J. Patera and H. Zassenhaus, Solvable Lie algebras of dimension $\leqslant 4$ over perfect fields, Linear Algebra Appl. 142 (1990), 1-17. MR1077969

[24] César Polcino Milies and Sudarshan K. Sehgal, An introduction to group rings, Algebra and Applications, vol. 1, Kluwer Academic Publishers, Dordrecht, 2002. MR1896125

[25] Daniel G. Quillen, On the associated graded ring of a group ring, J. Algebra 10 (1968), 411-418. MR0231919

[26] D. M. Riley and A. Shalev, Restricted Lie algebras and their envelopes, Canad. J. Math. 47 (1995), no. 1, 146-164. MR1319694

[27] David Riley and Hamid Usefi, The isomorphism problem for universal enveloping algebras of Lie algebras, Algebr. Represent. Theory 10 (2007), no. 6, 517-532. MR2350224

[28] David M. Riley, The dimension subalgebra problem for enveloping algebras of Lie superalgebras, Proc. Amer. Math. Soc. 123 (1995), no. 10, 2975-2980. MR1264829

[29] Csaba Schneider and Hamid Usefi, The isomorphism problem for universal enveloping algebras of nilpotent Lie algebras, J. Algebra 337 (2011), 126-140. MR2796067

[30] Helmut Strade and Rolf Farnsteiner, Modular Lie algebras and their representations, Monographs and Textbooks in Pure and Applied Mathematics, vol. 116, Marcel Dekker, Inc., New York, 1988. MR929682

[31] Moss E. Sweedler, Hopf algebras, Mathematics Lecture Note Series, W. A. Benjamin, Inc., New York, 1969. MR0252485

[32] Hamid Usefi, Isomorphism invariants of enveloping algebras, Noncommutative rings and their applications, 2015, pp. 253-265. MR3307403

[33] Albert Whitcomb, The group ring problem, ProQuest LLC, Ann Arbor, MI, 1968. Thesis (Ph.D.)-The University of Chicago. MR2611595 